\title{
Canadian Urological Association guideline on the management of non-muscle invasive bladder cancer
}

\author{
Bimal Bhindi, MD, CM, MSc, FRCSC*1; Ronald Kool, MD*2; Girish S. Kulkarni, MD, PhD, \\ FRCSC $^{3}$; D. Robert Siemens, MD, FRCSC ${ }^{4}$ Armen G. Aprikian, MD, FRCSC ${ }^{2}$; Rodney H. \\ Breau, MD, MSc, FRCSC ${ }^{5}$; Fadi Brimo, MD, FRCP ${ }^{6}$; Adrian Fairey, MD, FRCSC ${ }^{7}$; Christopher \\ French, MD, FRCSC ${ }^{8}$; Nawar Hanna, MD, MSc, FRCSC ${ }^{9}$; Jonathan I. Izawa, MD, FRCSC ${ }^{10}$; \\ Louis Lacombe, MD, FRCSC ${ }^{11}$; Victor McPherson, MD, MSc, FRCSC ${ }^{2}$; Ricardo A. Rendon, \\ MD, FRCSC ${ }^{12}$; Bobby Shayegan, MD, FRCSC ${ }^{13}$; Alan I. So, MD, FRCSC ${ }^{14}$; Alexandre R. \\ Zlotta, MD, PhD, FRCSC ${ }^{15}$; Peter C. Black, MD, FRCSC, FACS ${ }^{14}$; Wassim Kassouf, MD, CM, \\ FRCSC $^{2}$ \\ ${ }^{1}$ Section of Urology, Department of Surgery, University of Calgary, Calgary, AB, Canada; ${ }^{2}$ Division of Urology, \\ Department of Surgery, McGill University Health Centre, Montreal, QC, Canada; ${ }^{3}$ Division of Urology, Department \\ of Surgery, Princess Margaret Cancer Centre, University of Toronto, Toronto, ON, Canada; ${ }^{4}$ Department of \\ Urology, Queen's University, Kingston, ON, Canada; ${ }^{5}$ Division of Urology, University of Ottawa, Clinical \\ Epidemiology, The Ottawa Hospital Research Institute, Ottawa, ON, Canada; ${ }^{6}$ Department of Pathology, McGill \\ University Health Centre, Montreal, QC, Canada; ${ }^{7}$ Division of Urology, University of Alberta, Edmonton, AB, \\ Canada; ${ }^{8}$ Division of Urology, Department of Surgery, Memorial University of Newfoundland, St. John's, NL, \\ Canada; ${ }^{9}$ Department of Urology, Université de Montréal, Montreal, QC, Canada; ${ }^{10}$ Department of Surgery, \\ Division of Urology, Western University, London, ON, Canada; ${ }^{11}$ Department of Surgery, Faculty of Medicine, \\ Université Laval, Laval, QC, Canada; ${ }^{12}$ Department of Urology, Dalhousie University, Halifax, NS, Canada; \\ 12Division of Urology, McMaster University, Hamilton, ON, Canada; ${ }^{13}$ Department of Urologic Sciences, \\ University of British Columbia, Vancouver, BC, Canada; ${ }^{14}$ Division of Urology, Department of Surgery, Sinai \\ Health System and Department of Surgical Oncology, University Health Network, Toronto, ON, Canada \\ *Co-first authors with equal contributions
}

Cite as: Bhindi B, Kool R, Kulkarni GS, et al. Canadian Urological Association guideline on the management of non-muscle invasive bladder cancer. Can Urol Assoc J 2021 April 26; Epub ahead of print. http://dx.doi.org/10.5489/cuaj.7367

Published online April 26, 2021

Corresponding author: Dr. Wassim Kassouf, McGill University Health Centre, Montreal, QC, Canada; wassim.kassouf@muhc.mcgill.ca 


\section{Table of contents}

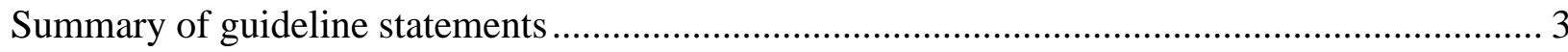

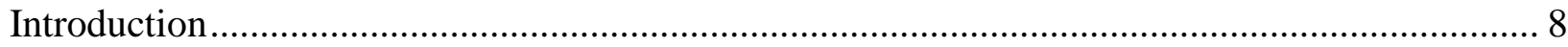

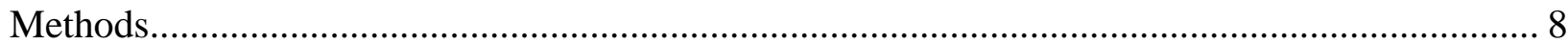

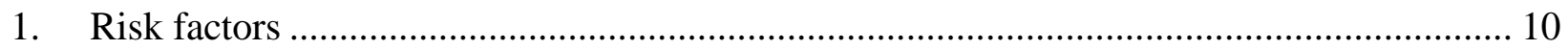

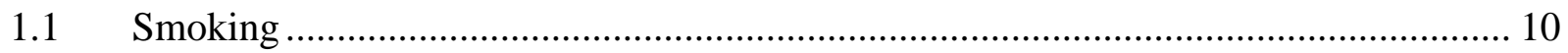

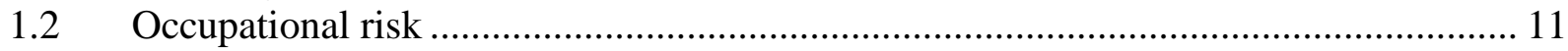

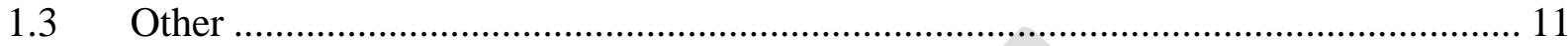

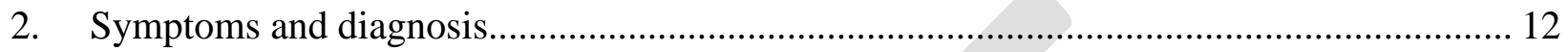

3. Prognostic factors for recurrence and progression...................................................... 14

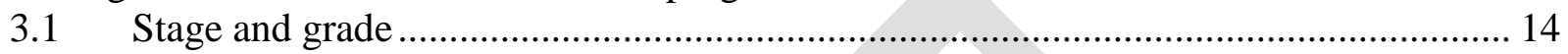

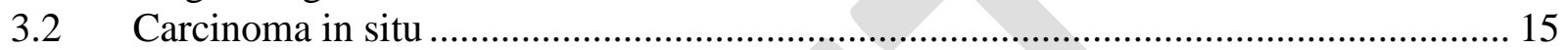

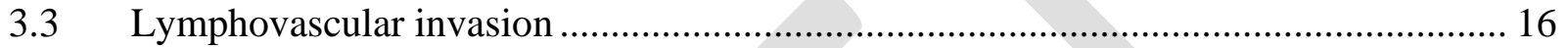

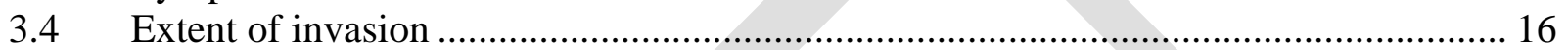

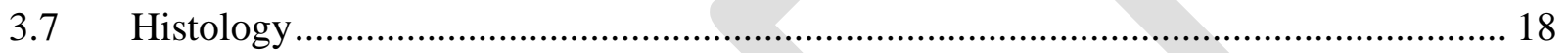

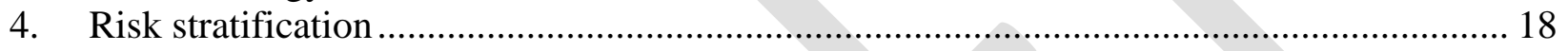

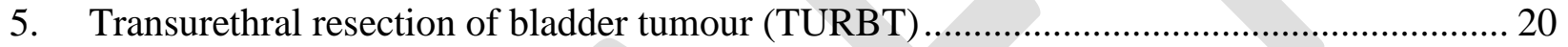

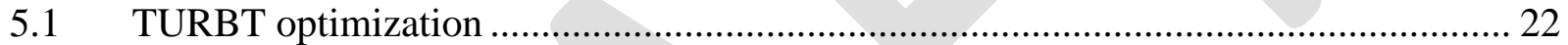

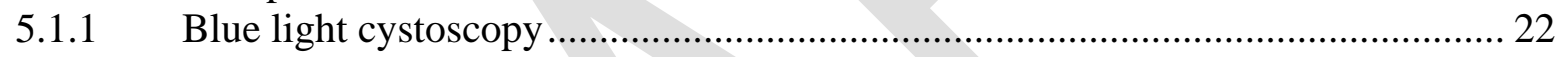

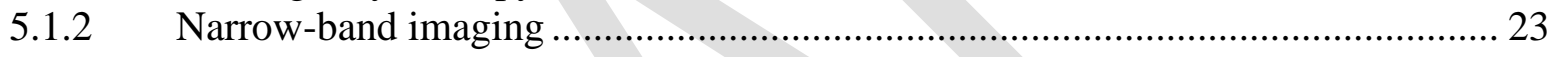

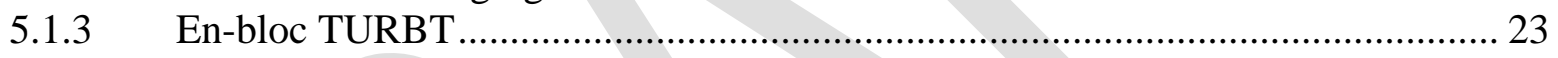

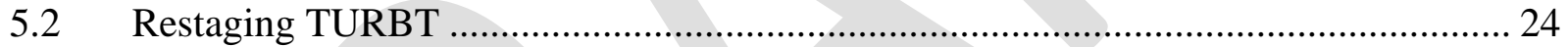

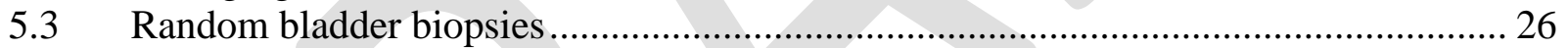

5.4 Prostatic urethral involvement (PUI) ............................................................ 27

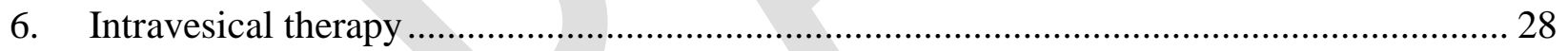

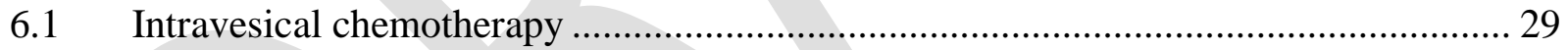

6.1.1 Single instillation of chemotherapy (SIC) post-TURBT ................................ 29

6.1.2 Adjuvant intravesical chemotherapy..................................................... 31

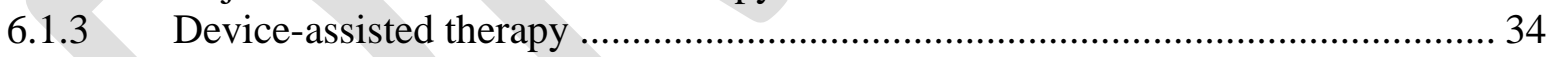

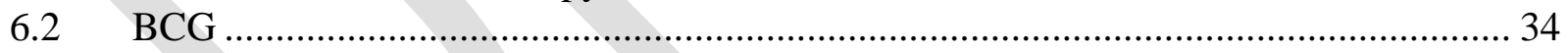

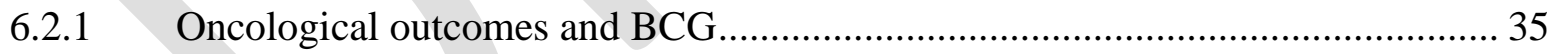

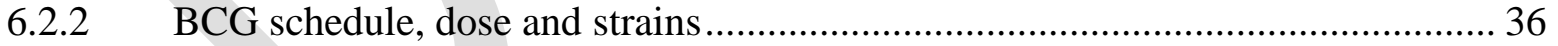

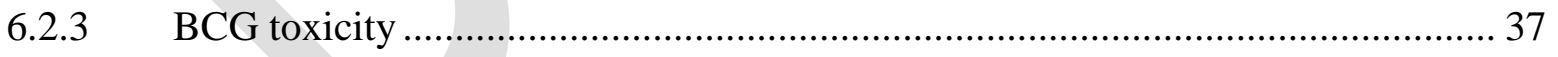

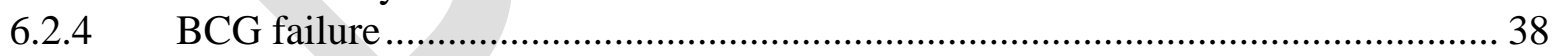

6.2.5 Management of BCG-unresponsive NMIBC ............................................. .40

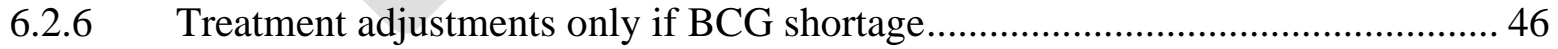

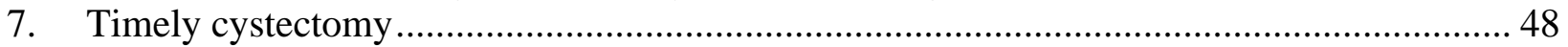

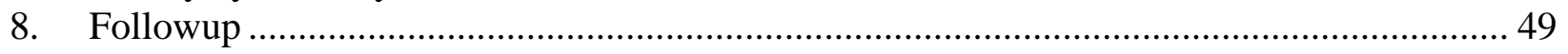

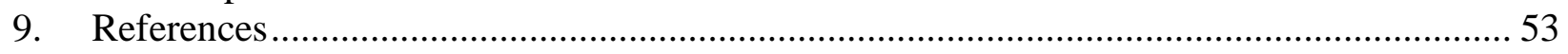




\section{Summary of guideline statements}

\section{Risk factors}

1. Former or current tobacco smoking is the most common risk factor associated with bladder cancer and smoking cessation should be encouraged in all patients (Level of Evidence [LE] 3; strong recommendation).

\section{Symptoms and diagnosis}

2. White-light cystoscopy (WLC) is recommended in the initial evaluation of patients suspected to have bladder cancer. Cystoscopy should be performed with a flexible cystoscope whenever available (LE 1; strong recommendation).

3. Urine cytology (voided or collected by bladder washing) is recommended as an adjunct to cystoscopy in patients suspected to have bladder cancer ( $L E 2$; strong recommendation).

4. Upper urinary tract imaging is recommended in the initial workup of patients suspected to have bladder cancer (LE 3; strong recommendation).

\section{Prognostic factors for recurrence and progression}

5. The most important prognostic factors for recurrence and progression of non-muscleinvasive bladder cancer (NMIBC) are stage and grade ( $L E 2)$. All patients with bladder cancer should be properly staged and, specifically for NMIBC, reporting grade is paramount for further management decisions ( $L E 2$; strong recommendation).

6. Other prognostic factors are age $>70 \mathrm{yr}$, large tumour size $(\geq 3 \mathrm{~cm})$, multiple tumours, the presence of concomitant carcinoma in situ (CIS), extensive invasion of the lamina propria, prior recurrence rates $>1$ per year and status at first assessment after transurethral resection of the bladder tumour (TURBT) ( $L E 2$ ), as well as lymphovascular invasion (LVI) (LE 3).

7. Aggressive histological variants such as micropapillary, plasmacytoid and sarcomatoid are associated with increased risk of under-staging and progression ( $L E 3)$. Pathological review, preferably by a dedicated uro-pathologist, should be considered in settings where variant histology is suspected or atypical tumours are seen during TURBT (e.g., sessile mass) (LE 3; weak recommendation).

\section{Risk stratification}

8. All patients with NMIBC should be stratified according to the risk of both recurrence and progression for adequate patient counselling and treatment planning (LE 2; strong recommendation). The modified CUA risk stratification system is a suitable tool for this purpose. 


\section{Transurethral resection}

9. Patients presenting with a bladder tumour should undergo initial TURBT for diagnostic confirmation and pathological evaluation (LE 2; strong recommendation).

10. Initial TURBT aims for complete tumour resection with sampling of the underlying detrusor muscle as the first step of curative-intent treatment of NMIBC (LE 2; strong recommendation). Patients with presumed low grade (LG) Ta or CIS might be spared from muscle sampling at initial TURBT (LE 3; weak recommendation).

11. When available, blue light cystoscopy (BLC) ( $L E$ 1; weak recommendation) or narrow band imaging ( $L E 2$; weak recommendation) can increase tumour detection at first TURBT and reduce recurrence risk.

12. A restaging TURBT should be performed in patients with T1 NMIBC, or when a complete resection was not achieved with the first TURBT (LE 2; strong recommendation). Restaging TURBT is not required in patients who will proceed to radical cystectomy (RC) based on the findings of the first TURBT.

13. In select cases of high grade (HG) Ta tumours (e.g., large and/or multiple tumours), a restaging TURBT can be considered ( $L E 3$; weak recommendation).

14. The suggested window for a restaging TURBT is within 6 weeks of the first resection ( $L E 3$; weak recommendation).

15. Patients presenting with a positive urine cytology, but normal appearing bladder at WLC and normal upper urinary tract imaging are at higher risk of harbouring occult CIS and should undergo random bladder biopsies (or use of BLC with directed biopsies) (LE 2; strong recommendation).

16. Biopsies or transurethral resection of the prostatic urethra should be included with random bladder biopsies in the presence of a positive bladder urine cytology, but normal appearing bladder at WLC and normal upper tract imaging (LE 3; strong recommendation).

17. Prostatic urethral biopsy (or transurethral resection) can also be considered in the presence of extensive bladder CIS or tumour at the bladder neck or trigone (LE 3; weak recommendation).

18. Patients with prostatic urethral involvement (PUI) with CIS restricted to the urethral mucosa can be managed conservatively with transurethral resection of the prostate (TURP) plus intravesical BCG ( $L E$ 3; weak recommendation). Repeat prostatic urethral biopsies after induction BCG should be considered ( $L E 3$; weak recommendation). $\mathrm{RC}$ can be discussed as an alternative option ( $L E 4$; weak recommendation).

19. In patients with HG T1 or CIS extending into the prostatic ducts, RC should be considered (LE 3; weak recommendation). TURP followed by intravesical BCG is an alternative option. In this instance, close followup with repeat prostatic urethral biopsies after induction BCG should be considered (LE 3; weak recommendation). 
20. In patients with prostatic stromal invasion, neoadjuvant cisplatin-based chemotherapy followed by $\mathrm{RC}$ is recommended ( $L E 3$; strong recommendation - refer to MIBC CUA guideline).

\section{Single instillation of chemotherapy (SIC) post-TURBT}

21. SIC (with mitomycin-C, epirubicin, doxorubicin, pirarubicin or gemcitabine) should be offered to all patients with presumed low-risk NMIBC at TURBT and should be administered within 24 hours after endoscopic resection (LE 1; strong recommendation).

22. SIC is recommended for intermediate-risk NMIBC and for patients with $\leq 1$ recurrence/year and EORTC recurrence score $<5$ (LE 1; strong recommendation). SIC should be discussed even when further adjuvant intravesical chemotherapy is planned (LE 2; weak recommendation).

23. The benefit of SIC in patients with high-risk NMIBC is unclear when intravesical Bacillus Calmette-Guérin (BCG) is planned as adjuvant treatment ( $L E 3$ ).

24. SIC should not be administered after extensive resection or when bladder perforation is suspected (LE 3; strong recommendation).

\section{Adjuvant intravesical chemotherapy}

25. Patients with intermediate-risk NMIBC should be considered for adjuvant induction intravesical chemotherapy ( $L E$ 1; strong recommendation) with subsequent maintenance for up to 1 year ( $L E 3$; weak recommendation), or induction BCG with maintenance therapy (refer to statement \#30).

26. Substratification of intermediate-risk patients with recurrent LG Ta NMIBC can be used to guide adjuvant treatment decisions ( $L E 3$; weak recommendation). For this purpose, 4 factors should be considered: number of tumours, size $(\geq 3 \mathrm{~cm})$, time to recurrence $(<1$ year) and frequency of recurrence ( $>1$ /year).

- Patients with low-intermediate-risk NMIBC (0 factors) may be treated similarly to low-risk patients, with SIC alone (LE 3; weak recommendation).

- Patients with high-intermediate-risk NMIBC ( $\geq 3$ factors) may be treated as highrisk patients with induction and maintenance $\mathrm{BCG}$ ( $L E 3$; weak recommendation).

27. Patients who develop recurrence during intravesical chemotherapy may be offered induction followed by maintenance BCG ( $L E 3$; weak recommendation).

28. Although intravesical chemotherapy through device-assisted therapy has shown promising results in small randomized controlled trials, further studies are needed to validate its routine clinical use.

\section{Adjuvant intravesical BCG}

29. In patients with high-risk NMIBC, BCG therapy with induction (weekly instillations for 6 weeks) followed by 3-year maintenance (weekly instillations for 3 weeks at 3, 6, 12, 18, 24, 
30 and 36 months) is the standard of care for reducing disease recurrence and progression rates (LE 1; strong recommendation).

30. When BCG is administered for intermediate-risk NMIBC, induction (weekly instillations for 6 weeks) followed by 1-year maintenance (weekly instillations for 3 weeks at 3, 6 and 12 months) is recommended (LE 1; strong recommendation).

31. $\mathrm{RC}$ with pelvic lymph node dissection is the standard of care for BCG-unresponsive bladder cancer in surgically fit patients ( $L E 3$; strong recommendation). For patients with BCGunresponsive CIS or $\mathrm{HG}$ Ta, a second-line bladder-preserving therapy can be considered before $\mathrm{RC}$ ( $L E 3$; weak recommendation).

32. Promising efficacy has been reported with intravenous pembrolizumab, intravesical oportuzumab monatox, nadofaragene firadenovec, and BCG plus N-803. These should be considered as potential options in patients with BCG-unresponsive CIS who are unfit for or refuse to undergo $\mathrm{RC}$ ( $L E$ 2; weak recommendation).

33. Alternative options such as sequential intravesical gemcitabine/docetaxel (induction plus maintenance) may be considered for patients with BCG-unresponsive disease who are unfit for or refuse to undergo $\mathrm{RC}$ ( $L E$ 3; weak recommendation). Additional alternatives may also include other combination intravesical therapy (e.g., sequential gemcitabine/mitomycin-C, $\mathrm{BCG}+$ interferon if available) or single-agent intravesical therapy (mitomycin-C, epirubicin, docetaxel, gemcitabine) ( $L E 3$; weak recommendation).

34. Clinical trials may be considered for BCG-unresponsive patients who are unfit for or refuse to undergo RC.

\section{Treatment adjustments only if $B C G$ shortage}

35. For patients with intermediate-risk NMIBC during BCG shortage, intravesical chemotherapy is recommended as the first-line option. If $\mathrm{BCG}$ is planned as a second-line therapy for this population, induction might be administered with reduced dosing (1/2 or $1 / 3$ dose) and maintenance can be omitted ( $L E 3$; weak recommendation).

36. For patients with high-risk NMIBC, full BCG schedule (induction followed by maintenance) is recommended ( $L E$ 1; strong recommendation). Only during BCG shortage, when full dose is not possible due to limited supply, dose reduction to $1 / 2$ or $1 / 3$ might be considered, while maintenance can be reduced to one year ( $L E 3$; weak recommendation).

37. When BCG is unavailable, single agent chemotherapy (e.g., mitomycin-C, gemcitabine) or sequential combination of intravesical chemotherapy (e.g., gemcitabine/docetaxel) is recommended with induction followed by monthly maintenance for up to one year ( $L E 3$; weak recommendation). 


\section{Timely cystectomy}

38. Upfront RC should be considered for patients with large volume, diffuse, endoscopically unresectable NMIBC (LE 3; strong recommendation)

39. Upfront RC should be offered to patients with HG T1 disease with additional adverse tumour pathological features including: variant histology (e.g., micropapillary, plasmacytoid, sarcomatoid), extensive invasion of the lamina propria or invasion into or beyond the muscularis mucosa (T1b/c), presence of LVI, concomitant CIS in the bladder or prostatic urethra, multiple and large $(\geq 3 \mathrm{~cm}$ ) tumours, or persistent $\mathrm{HG}$ T1 upon restaging TURBT ( $L E$ 3 ; strong recommendation).

\section{Followup}

40. The first surveillance cystoscopy is recommended for all patients at 3 months after TURBT (LE 2; strong recommendation).

41. A risk-based surveillance strategy should be used in patients with no evidence of recurrence at the 3-month cystoscopy:

- Low-risk patients might be followed with cystoscopy at 1 year and then yearly for 5 years (LE 3; weak recommendation). Urine cytology is not necessary in the followup of low-risk patients ( $L E 4$; weak recommendation).

- Intermediate-risk patients should be followed with cystoscopies and urine cytology every 3-6 months in the first 2 years, every 6-12 months in the $3^{\text {rd }}$ year, and annually thereafter ( $L E 3$; weak recommendation).

- High-risk patients should be followed with cystoscopies and urine cytology every 3-4 months during the first 2 years, every 6 months during years 3 and 4, and annually thereafter ( $L E 3$; weak recommendation).

42. Upper tract imaging is recommended with random bladder/prostatic urethral biopsies (or use of BLC with directed biopsies) if positive urine cytology with normal WLC is found during surveillance ( $L E 3$; weak recommendation).

43. Upper tract imaging is recommended in the first year and every 2 years thereafter for highrisk patients ( $L E 3$; weak recommendation).

44. Fulguration under local anesthesia might be considered for small $(<5 \mathrm{~mm})$ papillary tumours and negative cytology in patients with a prior history of papillary urothelial neoplasm of low malignant potential or LG Ta NMIBC (LE 3; weak recommendation). 


\section{Introduction}

Bladder cancer is the fifth most common cancer worldwide ${ }^{1}$. In Canada, the estimated incidence for 2020 was of 12,200 new cases, with 2,600 disease-related deaths during that same year². Canadian population-based studies suggest that incidence was stable from 2010 until $2015^{3}$. More recent estimates, however, have shown an increase in incidence rates from 21.8 to 25.0 cases per 100,000 from 2018 to $2020^{2}$. Bladder cancer is the fourth most common cancer among men, accounting for $8.1 \%$ of all cancer diagnoses and the male to female ratio is $3: 1$. It is considered a disease of the elderly with an average age at diagnosis of 73 years. It is more prevalent in resourced and industrialized countries with the highest incidence rates in North America, Europe and Western Asia ${ }^{4}$.

Approximately $75 \%$ of all bladder tumours are diagnosed at early stages, classified as non-muscle invasive bladder cancer (NMIBC). NMIBC corresponds to clinical tumour stages Tis (carcinoma in situ), Ta and T1 according to the $8^{\text {th }}$ edition of the American Joint Committee on Cancer and the Union for International Cancer Control classification (Table 1) ${ }^{5}$. These tumours are either confined to the mucosal layer or invade the submucosal layer (lamina propria) of the bladder wall without invading the underlying detrusor muscle (muscularis propria). Despite having a good prognosis, NMIBC is associated with high recurrence rates and some patients will progress to muscle-invasive disease (T2 or higher). The remaining $25 \%$ of bladder tumours are muscle-invasive (MIBC) upfront at diagnosis (cT2-T4) and/or metastatic (cM1); associated with higher mortality rates. The management of MIBC and metastatic bladder cancer is addressed in separate CUA guidelines ${ }^{6,7}$. In addition, estimated life cost of bladder cancer is the highest among all malignancies because of the need for close surveillance and the high recurrence and progression rates, which often necessitate multiple lines of therapy ${ }^{8,9}$.

\section{Table 1. Bladder TNM classification - AJCC UICC, $8^{\text {th }}$ edition}

\begin{tabular}{|l|l|}
\hline \multicolumn{2}{|l|}{ T - primary tumour } \\
\hline TX & Primary tumour cannot be assessed \\
\hline T0 & No evidence of primary tumour \\
\hline Ta & Noninvasive papillary carcinoma \\
\hline Tis & Urothelial carcinoma in situ: 'flat tumour' \\
\hline T1 & Tumour invades lamina propria (subepithelial connective tissue) \\
\hline T2 & Tumour invades muscularis propria (detrusor muscle) \\
\hline T3 & $\begin{array}{c}\text { Tumour invades perivesical soft tissue } \\
\text { T3a }\end{array}$ \\
T3b & Macroscopic invasion \\
\hline
\end{tabular}




\begin{tabular}{|c|c|}
\hline $\begin{array}{l}\mathrm{T} 4 \mathrm{a} \\
\mathrm{T} 4 \mathrm{~b}\end{array}$ & $\begin{array}{l}\text { Extravesical tumour invades any of the following: prostatic stroma, seminal } \\
\text { vesicles, uterus, vagina, pelvic wall, abdominal wall } \\
\text { Tumour invades prostatic stroma, seminal vesicles, uterus or vagina } \\
\text { Tumour invades pelvic wall or abdominal wall }\end{array}$ \\
\hline \multicolumn{2}{|c|}{$\mathbf{N}$ - regional lymph nodes } \\
\hline $\mathrm{Nx}$ & Regional lymph nodes cannot be assessed \\
\hline N0 & No lymph node metastasis \\
\hline N1 & $\begin{array}{l}\text { Single regional lymph node metastasis in the true pelvis (perivesical, obturator, } \\
\text { external iliac, or sacral) }\end{array}$ \\
\hline $\mathrm{N} 2$ & $\begin{array}{l}\text { Multiple regional lymph node metastasis in the true pelvis (perivesical, obturator, } \\
\text { external iliac, or sacral) }\end{array}$ \\
\hline $\mathrm{N} 3$ & Lymph node metastasis to common iliac lymph node(s) \\
\hline \multicolumn{2}{|c|}{ M-distant metastasis } \\
\hline M0 & No distant metastasis \\
\hline $\begin{array}{l}\text { M1 } \\
\text { M1a } \\
\text { M1b }\end{array}$ & $\begin{array}{l}\text { Distant metastasis } \\
\text { Nonregional lymph nodes (beyond the common iliac vessels) } \\
\text { Other distant metastasis }\end{array}$ \\
\hline
\end{tabular}

\section{Methods}

For this updated version, a non-systematic literature review was performed in Medline and PubMed using keywords and MESH terms. Previously published sections of the 2015 version $^{10}$ were reorganized and updated, adding recent literature from October $14^{\text {th }}, 2015$ until February $13^{\text {th }}, 2021$.

Statements for interventions and prognosis here provided were assigned a level of evidence (LE) based on a modified version of the 2009 Oxford Centre for Evidence-based Medicine classification which has been used by the Joint Consultation of the Société Internationale d'Urologie and International Consultation on Urological Disease (SIU-ICUD) on Bladder Cancer, published in 2017 (Table 2) ${ }^{11}$. Moreover, recommendation statements were classified as strong versus weak using a modified GRADE approach ${ }^{12}$. The strength of each recommendation was judged based on the quality of evidence, the estimated magnitude, precision and consistency of the effect, and the balance between potential benefits and harms, resource use, value judgments, and patient preferences. Other society NMIBC guidelines published by the European Association of Urology (EAU), the American Urological Association (AUA), the National Institute for Health and Care Excellence (NICE) and the National Comprehensive Cancer Network (NCCN) were used for comparison ${ }^{13-16}$. 


\begin{tabular}{|c|c|c|}
\hline $\begin{array}{l}\text { Level of } \\
\text { evidence }\end{array}$ & Studies on intervention & Studies on prognosis \\
\hline 1 & $\begin{array}{l}\text { Meta-analysis of randomized controlled trials. } \\
\text { Good-quality randomized controlled trial }\end{array}$ & Meta-analysis of inception cohort studies \\
\hline 2 & $\begin{array}{l}\text { Low-quality randomized controlled trial. } \\
\text { Good-quality prospective cohort study }\end{array}$ & Inception cohort study \\
\hline 3 & $\begin{array}{l}\text { Good-quality retrospective case-control } \\
\text { or cohort study }\end{array}$ & $\begin{array}{l}\text { Cohort study or control arm of } \\
\text { randomized controlled tria }\end{array}$ \\
\hline 4 & Expert opinion & $\begin{array}{l}\text { Case series, case-control study, or poor- } \\
\text { quality prognostic cohort study }\end{array}$ \\
\hline
\end{tabular}

\section{Risk factors}

- Former or current tobacco smoking is the most common risk factor associated with bladder cancer and smoking cessation should be encouraged in all patients (LE 3; strong recommendation).

\subsection{Smoking}

Among several well-established risk factors, tobacco use is the most remarkable one, being the attributable cause of bladder cancer in $50 \%$ of the cases ${ }^{17,18}$. In 2016, Cumberbatch et al conducted a meta-analysis including 83 studies and found a relative risk for bladder cancer of 3.47 and 2.04 for current and ex-smokers, respectively, compared to those who have never smoked $^{19}$. Smoking history is associated with a three-fold increase in the risk of developing bladder cancer, and the risk is linearly associated with both the quantity and duration of exposure, in packs-years ${ }^{20,21}$. The benefit of smoking cessation is based on weak evidence and prospective evaluation is lacking ${ }^{19,22,23}$. Nevertheless, smoking cessation should be highly encouraged after a bladder cancer diagnosis, with potential additional benefits of reducing the risk of developing other malignancies (e.g., lung cancer) and cardiovascular disease (LE 3; strong recommendation $)^{23,24}$.

Electronic cigarette (e-cigarette) use may also be associated with a risk of developing bladder cancer as similar carcinogens have been detected in the urine of both regular and ecigarette users (e.g., o-toluidine and 2-naphthylamine ${ }^{25}$. A recent systematic review on 22 studies showed that a total of 40 compounds linked to potential development of bladder cancer were identified in the urine of e-cigarette smokers, out of which 12 are classified as group 1 (carcinogenic to humans) according to the International Agency for Research on Cancer $(\text { IARC })^{26}$. To date, however, defining a direct causal link between e-cigarettes and bladder 
cancer has been challenging due to current study limitations such as dual exposure (patients exposed to multiple tobacco products) and need for longer followup. Patients should be counseled regarding a potential risk of e-cigarettes and bladder cancer.

The role of cannabis use and bladder cancer was investigated in a small study that suggested a potential risk increase, based on a higher proportion of users among bladder cancer patients compared to controls ${ }^{27}$. This study, however, was limited by small sample size and selection bias $^{27}$. More recently, a large cohort study with more than 84,000 men investigated the incidence of bladder cancer among tobacco and cannabis users ${ }^{28}$. Cannabis use was associated with bladder cancer risk reduction of $45 \%$ (HR $0.55,95 \%$ CI $0.31-1.00$ ) when adjusting for other confounders ${ }^{28}$. Although a potential antiproliferative role of cannabinoids in urological cancers has been proposed, further prospective studies are needed to validate this hypothesis ${ }^{29}$.

\subsection{Occupational risk}

Occupational exposure is the second most common cause of bladder cancer and was reported as being the attributable cause of approximately $8-10 \%$ of cases during the 1980 's. With workplace legislation, this estimate has recently decreased to approximately $5 \%{ }^{30-32}$. The most important carcinogens are those associated with the rubber (2-napthylamine), plastic (1,1-dichloroethane, 4,4'-methylenebis(2-chloroaniline) - MBOCA - and 4,4'-methylenedianiline) and dye (aromatic amines and polycyclic aromatic hydrocarbons) industries ${ }^{33,34}$. In a meta-analysis with 263 studies and more than 30 million people, 42 occupations were associated with increased risk of bladder cancer, with the greatest risk among patients working in factories (RR, 16.6 [95\% CI, 2.1-131.3]) and in the dye industry (RR, 13.4 [95\% CI, 1.5-48.2]) $)^{32}$.

\subsection{Other}

Advanced age is an independent risk factor for developing bladder cancer and incidence increases remarkably after the age of 65 , reaching its peak at the age of $84^{35}$. Median age at bladder cancer diagnosis is 73 years ${ }^{36}$. Additionally, over $80 \%$ of bladder cancer related deaths occur in patients over 65 years old ${ }^{37}$. Sex is a well-recognized risk factor, as male to female bladder cancer incidence ratio is 3:1. Gender and sex disparities are multifactorial and have been related in the past to unequal exposure to carcinogens (e.g., tobacco, occupational exposure), hormonal factors and delays in diagnosis ${ }^{38-41}$. Moreover, women also tend to present with more aggressive disease at diagnosis (e.g., a higher proportion of MIBC) and a higher proportion of certain variant histologies (e.g., squamous cell carcinoma), which results in worse oncological outcomes $^{42,43}$. Particularly for NMIBC, woman seem to harbour a higher risk of disease recurrence when treated with intravesical Bacillus Calmette-Guérin (BCG), according to a metaanalyses with 23,754 patients $^{44}$. Currently, other biological factors are being studied, with the aim to understand additional reasons for sex disparities in bladder cancer, and ultimately to 
optimize health care delivery, encourage earlier diagnosis and adequate treatment to mitigate this discrepancy $^{45-48}$.

Previous pelvic irradiation for other cancers has been associated with second malignancies, including bladder cancer. For example, after radiation for prostate cancer, the hazard ratio for developing bladder cancer is 1.67 (95\% CI 1.55-1.80), with median time from exposure to bladder cancer diagnosis of 58 months [IQR 31-93] $]^{49,50}$. Bladder cancer risk is increased in patients exposed to arsenic (e.g., in drinking water) ${ }^{18}$. The association between other dietary factors and bladder cancer is controversial and require further investigation ${ }^{18,51}$. Cyclophosphamide, phenacetin, and chlornaphazine are listed carcinogens that can cause bladder cancer, according to the IARC ${ }^{52}$. Other medications, such as pioglitazone, may be associated with bladder cancer risk, although evidence to date remains controversial ${ }^{53-56}$.

Genetic susceptibility to bladder cancer has been demonstrated. Inherited mutations in carcinogen-detoxification genes, such as NAT2 and GSTM1 can lead to higher exposure to carcinogens (e.g., cigarette components) and higher risk of bladder cancer ${ }^{57}$. Lynch Syndrome (LS), also known as hereditary non-polyposis colorectal cancer, is caused by an autosomal dominant germline mutation in mismatch repair genes ${ }^{58}$. LS is related to both colonic and extracolonic cancers, including upper tract urothelial carcinoma (UTUC). A study by Skeldon et al suggested that LS is also associated with bladder cancer, as more than $6 \%$ of patients carrying a specific mutation in the MSH2 gene were diagnosed with the disease ${ }^{59}$. Therefore, awareness of LS among urologists is important for appropriate screening and counselling of bladder cancer patients $^{60}$.

Chronic inflammation to the bladder mucosa (e.g., recurrent urinary tract infections and indwelling catheters) is also associated with higher bladder cancer risk ${ }^{18}$. Additionally, infection with Schistosoma haematobium is a well-recognized cause of chronic bladder inflammation and is associated with increased risk of squamous cell carcinoma (SCC) of the bladder ${ }^{18,61}$. Schistosomiasis-induced bladder cancer is more common in developing countries such as Egypt, but its incidence has been decreasing in the last 20 years, such that urothelial carcinoma (UC) has replaced SCC as the most common histology of bladder cancer in these countries as well ${ }^{62}$.

\section{Symptoms and diagnosis}

- White-light cystoscopy (WLC) is recommended in the initial evaluation of patients suspected to have bladder cancer. Cystoscopy should be performed with a flexible cystoscope whenever available ( $L E 1$; strong recommendation).

- Urine cytology (voided or collected by bladder washing) is recommended as an adjunct to cystoscopy in patients suspected to have bladder cancer ( $L E 2$; strong recommendation).

- Upper urinary tract imaging is recommended in the initial workup of patients suspected to have bladder cancer ( $L E 3$; strong recommendation). 
Workup for suspected bladder cancer includes a complete history, and a focused physical exam, followed by cystoscopy and imaging. Ultrasound is operator-dependent, and historically, its sensitivity and specificity for detecting bladder cancer is $91 \%$ and $79.3 \%$, respectively, with overall accuracy of $88 \%{ }^{63}$. Accuracy, however dramatically decreases for defining lesions < $5 \mathrm{~mm}$ and its utility for staging bladder cancer and ruling out UTUC is limited ${ }^{64}$. Cross-sectional imaging is, therefore, recommended in the initial workup of bladder cancer, especially in patients presenting with gross hematuria where contrast-enhanced computerized tomography (CT) with delayed images is the first-line option, showing high sensitivity (85\%) and specificity (94\%) in detecting bladder tumours with a diagnostic accuracy of $90 \%{ }^{65}$. Despite its limitation in determining depth of invasion (NMIBC versus MIBC tumours), CT is important to assess for concurrent UTUC (present in approximately $2 \%$ of patients with bladder cancer), hydroureteronephrosis (associated with higher T-stage bladder cancer), and to rule out metastatic disease (LE 3; strong recommendation) ${ }^{66,67}$.

Recently, a standardized evaluation of local bladder tumour staging through multiparametric magnetic resonance (MRI) was proposed - the Vesical Imaging-Reporting and Data System (VI-RADS) ${ }^{68}$. The objective was to mitigate staging errors and optimize treatment of bladder cancer by improving the accuracy in predicting muscularis propria invasion at the time of diagnosis ${ }^{68}$. In a meta-analysis of 6 studies, VI-RADS was able to predict muscle invasion with $83 \%$ sensitivity and $90 \%$ specificity in patients undergoing transurethral resection (TUR) and/or radical cystectomy (RC) ${ }^{69}$. Despite promising results, VI-RADS still needs validation in larger prospective studies to determine the added value compared to TUR alone and the ideal timing for the MRI (before versus after initial TUR).

WLC is the cornerstone for establishing bladder cancer diagnosis. According to randomized controlled trials (RCTs), flexible cystoscopy in males has been shown to reduce pain and improve patients' acceptance of this procedure (LE 1) ${ }^{70,71}$. Likewise, a recent RCT demonstrated lower pain levels during flexible, compared to rigid cystoscopy, in female patients $^{72}$. Therefore, initial WLC should be done using gel lubrication and a flexible cystoscope when available (LE 1; strong recommendation). On the other hand, the benefit of using an anesthetic lubricant for pain relief during flexible cystoscopy, in comparison to plain lubricant, is currently not consistent in the literature ${ }^{73,74}$. A recent randomized study also showed that the "bag squeeze" technique (applying pressure to the saline bag during cystoscopy at the level of the membranous / prostatic urethra) was significantly associated with a lower mean pain score during the procedure when compared to controls (1.91 versus 3.39, respectively; $p<0.001)^{71}$.

Either voided or bladder washing urine cytology should be performed as an adjunct to cystoscopy in the initial diagnosis of NMIBC (LE 2; strong recommendation) ${ }^{75,76}$. Overall, the sensitivity of a positive urine cytology in detecting bladder cancer is low, ranging from $40 \%$ to 
$60 \%$, but is as high as $70-90 \%$ for high grade (HG) tumours and carcinoma in situ (CIS) ${ }^{77-80}$. Specificity, however, is high (around $>95 \%$ ) leading to a very low rate of false-positive results ${ }^{81}$. Novel biomarkers for detecting bladder cancer at initial diagnosis or surveillance are being studied. To date, however, biomarkers are not yet able to outperform or replace cystoscopy.

3. Prognostic factors for recurrence and progression

- The most important prognostic factors for recurrence and progression of NMIBC are stage and grade ( $L E 2)$. All patients with bladder cancer should be properly staged and, specifically for NMIBC, reporting grade is paramount for further management decision ( $L E 2$; strong recommendation).

- Other prognostic factors are age $>70 \mathrm{yr}$, large tumour size $(\geq 3 \mathrm{~cm})$, multiple tumours, the presence of concomitant CIS, extensive invasion of the lamina propria, prior recurrence rates $>1$ per year and status at first assessment after TURBT ( $L E 2$ ), as well as lymphovascular invasion (LVI) ( $L E ~ 3)$.

- Aggressive histological variants such as micropapillary, plasmacytoid and sarcomatoid are associated with increased risk of under-staging and progression ( $L E 3$ ). Pathological review, preferably by a dedicated uro-pathologist, should be considered in settings where variant histology is suspected or atypical tumours are seen during TURBT (e.g., sessile mass) (LE 3; weak recommendation)

\subsection{Stage and grade}

Stage and grade are the most important prognostic factors for predicting recurrence and progression of NMIBC ${ }^{82,83}$. A large retrospective study by Millan-Rodriguez et al with 1,529 patients treated for primary NMIBC showed that, when adjusted for other confounders in a multivariate model (including stage), grade was the most important factor associated with both progression (OR 19.9, 95\% CI 2.6 - 150.0) and disease-specific mortality (OR 14.0, 95\% CI 1.8 $-109.0)^{84}$. Regarding time to progression, the presence of HG disease was associated with a higher hazard ratio compared to stage (2.67 [95\% CI 1.99 - 3.59] versus 2.19 [95\% CI 1.67 2.86], respectively), resulting a higher weight for grade in the European Organisation for Research and Treatment of Cancer (EORTC) risk calculation tables for recurrence and progression (LE 2$)^{83}$. Therefore, all bladder cancer patients should be properly staged and, specifically for NMIBC, reporting grade is considered paramount for further management decision (LE 2; strong recommendation).

In 1973 the World Health Organization (WHO) defined a grading system for pathologists to report, dividing NMIBC in three categories according to levels of cell differentiation: grades 1, 2 and 3. Despite well-established criteria, difficulties in distinguishing grade 2 from grades 1 and 3 resulted in the middle category being overreported ${ }^{85}$. To address this issue, the WHO and the International Society of Urologic Pathologists (ISUP) updated this classification in 2004, 
which is to date the most widely adopted grading system in North America. It classifies tumours in three categories: papillary urothelial neoplasm of low malignant potential (PUNLMP), lowgrade (LG) and HG. The same grading system was reviewed without major changes in 2016. With the 2004/2016 WHO/ISUP system, 30-40\% of the former grade 2 lesions with bland cytological features were re-classified as LG, while the other $60-70 \%$ were re-classified as $\mathrm{HG}^{86}$.

In a recent systematic review including 20 studies, the interobserver reproducibility of the 2004/2016 WHO/ISUP system by pathologists was low (43-66\%) although higher when compared to the previous 1973 version $^{86}$. Moreover, when PUNLMP and LG tumours were considered together, reproducibility increased from $71-82 \%$ to $86-90 \%$. In that same study, recurrence rates among 624 patients with PUNLMP was $12 \%$. Importantly, a comparable recurrence rate $(9 \%)$ was reported among 1,303 patients with G1 tumours ${ }^{86}$. Progression of PUNLMP is rare (1.7\%), and this entity should be followed similarly to LG tumours (LE 3; weak recommendation $)^{86}$.

Approximately $70 \%$ of newly diagnosed NMIBC is detected at stage $\mathrm{Ta}^{87}$. Among Ta tumours, the vast majority are LG, with HG Ta tumours being diagnosed in only $7 \%$ of cases, while 5-10\% of Ta tumours harbour concomitant CIS ${ }^{88}$. Patients with single papillary LG Ta tumours measuring $<3 \mathrm{~cm}$ have the most favourable prognosis, with 5-year recurrence and progression risks of $21-31 \%$ and $0.8-3.8 \%$, respectively ${ }^{89}$. On the other hand, HG Ta tumours have a $43.5 \%$ risk of recurrence ( $28.8 \%$ within the first year), and a risk of progressing to $\geq \mathrm{pT} 1$ and $\geq \mathrm{pT} 2$ of $7.4 \%$ and $3.2 \%$, respectively, at a median followup of 44.5 months $^{90,91}$. Stage T1 tumours represent $20 \%$ of $\mathrm{NMIBC}^{87}$. In addition to a recurrence rate of $41.8 \%$ (95\% CI $398.5-$ 44.9), $20.2 \%$ (95\% CI 17.6 - 22.7) of HG T1 tumours progress to muscle invasion at 5 years according to a meta-analysis ${ }^{92}$. Stage $\mathrm{T} 1$ tumours are almost always HG, and only $2 \%$ have been classified as LG. Additionally, retrospective studies have shown that patients with these rare LG T1 tumours experienced similar oncological outcomes when compared to HG T1 patients, suggesting that the impact of grade within the spectrum of $\mathrm{T} 1$ disease remains questionable ${ }^{93-95}$.

\subsection{Carcinoma in situ}

Concomitant CIS is diagnosed in up to $19 \%$ of NMIBC cases ${ }^{96}$. This entity is described as a flat lesion confined to the bladder mucosa and is HG by definition ${ }^{97}$. If treated only with TURBT, CIS recurrence can be as high as $90 \%$ while progression to muscle-invasive disease can occur in $54-80 \%$ of patients ${ }^{98,99}$. CIS is rarely found in isolation, with the majority of cases $(90 \%)$ being found in association with papillary or nodular bladder tumours ${ }^{87,100}$. CIS can be classified as primary (no previous history of bladder cancer), which carries the best prognosis, concomitant (with a papillary or nodular tumour) or secondary (new lesion diagnosed during followup) ${ }^{101,102}$. CIS is also considered a field disease, as it can affect multiple areas in the bladder, the upper urinary tract, and the urethra ${ }^{103}$. Concomitant CIS has prognostic importance; it is significantly associated with risk of progression (OR 2.1,95\% CI 1.1 - 4.0) and disease-specific mortality 
(OR 3.0, 95\% CI 1.4 - 6.6) (LE 2) ${ }^{84}$. Therefore, erythematous areas suspicious for CIS should be biopsied during cystoscopy / TURBT (LE 2; strong recommendation).

\subsection{Lymphovascular invasion}

Initial series evaluating LVI for NMIBC have shown inconsistent results regarding its association with oncologic outcomes ${ }^{104-106}$. A prospective study by Cho et al showed LVI was a significant predictor for recurrence and progression, and was negatively associated with metastasis-free survival, but these results were limited by potential selection bias and short followup $^{107}$. A meta-analysis by Kim et al in 2014 with 3,905 patients taken from 16 retrospective studies showed the association of LVI with upstaging in RC specimens (OR 2.21, 95\% CI 1.44 - 3.39), recurrence-free survival (RFS) (HR = 1.47, 95\% CI $1.24-1.74$ ) and progression-free survival (PFS) $(\mathrm{HR}=2.28,95 \% \mathrm{CI} 1.45-3.58)$ when detected in TURBT specimens of patients with NMIBC ${ }^{108}$. In a meta-analysis with 15,215 patients designed to identify prognostic factors for HG T1 disease, Martin-Doyle et al showed that LVI was significantly associated with RFS, PFS and disease-specific survival (DSS) when including studies with at least $75 \%$ of patients with HG T1 tumours (LE 3) ${ }^{92}$. When analysis was performed on studies with $100 \%$ of HG T1 tumours, statistical significance was not maintained. Importantly, LVI was associated with deep invasion into the lamina propria, which was the most important adverse prognostic factor. Based on these findings, the authors questioned the independent role of LVI in the prognosis of HG T1 disease, suggesting it could ultimately reflect the extent of invasion into the lamina propria ${ }^{92}$. More recently, however, another meta-analysis with 33 retrospective studies and 6,194 patients showed that LVI was present in 15\% of TURBT NMIBC specimens (LE 3) ${ }^{109}$. In this study, LVI was a significant factor for recurrence [HR 1.97, 95\% CI 1.47 - 2.62], progression [HR 2.95, 95\% CI 2.11 - 4.13] and upstaging after RC, particularly for cT1 (LE 3) ${ }^{109}$.

Pathological evaluation of LVI may be limited if only hematoxylin \& eosin staining is used and the use of immunohistochemistry is encouraged in challenging cases in order to differentiate LVI from retraction artifact (LE 3; weak recommendation) ${ }^{110}$. Although it has not yet been integrated in the major risk stratification tools for NMIBC, LVI may be considered as an independent marker of worse prognosis in patients with high-risk NMIBC (LE 3 / weak recommendation).

\subsection{Extent of invasion}

The extent of invasion of T1 tumours is also associated with prognosis. It has been evaluated using two different criteria: 1) Micrometric: which evaluates the millimetric extent of invasion into the lamina propria, either in depth, or as a linear measurement of the invasive cancer focus (single largest focus or in aggregate); and 2) Microanatomic: which evaluates the level of invasion in relation to the muscularis mucosa (T1a - no muscularis mucosa invasion, $\mathrm{T} 1 \mathrm{~b}-$ 
invasion at the level of the muscularis mисовa and T1c - invasion beyond the muscularis тисоs $a)^{111-113}$. Although no single approach has been universally adopted, the micrometric method has the advantage of not being limited by the anatomical variability of muscularis mucosa (which may be discontinuous or absent, limiting the applicability of the microanatomic approach in daily practice). On the other hand, no single cut-off of either depth or length of invasive focus has been consistently demonstrated to be prognostic yet. A study by van Rhijn et al with 134 patients with newly diagnosed bladder cancer, identified a significant association between extensive T1 invasion of the lamina propria and progression [HR 3.0, 95\% CI $1.5-$ 5.90; $p=0.001]$ and DSS [HR 2.7, 95\% CI 1.1 - 6.8; p = 0.032]. Importantly, assessment of microscopic versus extensive $\mathrm{T} 1$ invasion was possible in $100 \%$ of the cases ${ }^{112}$. This was later confirmed in a multicentric study with 601 patients with primary pT1 bladder tumours in which extensive lamina propria invasion (but not muscularis mucosa level of invasion) was independently associated with PFS [HR 3.8, 95\% CI 2.3 - 6.0; $<<0.001]$ and DSS [HR 2.7,95\% CI $1.6-4.8 ; \mathrm{p}<0.001]^{113}$. Notably, the meta-analysis by Martin-Doyle showed that extensive invasion of the lamina propria was considered the most significant prognostic factor for HG T1 patients $(\mathrm{HR}=3.34,95 \% \mathrm{CI} 2.04-5.49, \mathrm{p}<0.001)^{92}$. More recently, a systematic review and meta-analysis with 36 studies and 6,781 patients confirmed both deep invasion into the lamina propria and invasion to the muscularis mucosa to be associated with disease recurrence and progression in NMIBC when adjusted for other potential confounders such as tumour grade, CIS and multifocality ${ }^{114}$. This meta-analysis included 4 prospective studies, out of which 3 showed a significant prognostic role of deep lamina propria invasion in both recurrence and progression during followup (LE 2) $)^{115-117}$

\subsection{Tumour burden (size and multifocality)}

The number and size of tumours are also important prognostic factors for recurrence in NMIBC ${ }^{18-121}$. Recurrence rates are significantly higher for NMIBC patients with multiple (OR $1.9,95 \% \mathrm{CI}=1.1-3.2)$ and large $(\geq 3 \mathrm{~cm})$ tumours $(\mathrm{OR} 1.7,95 \% \mathrm{CI}=1.0-3.0)(\mathrm{LE} 2)^{84}$. Both variables were included in the EORTC and the Spanish Urological Club for Oncological Treatment (CUETO) risk stratification tools ${ }^{83,122}$. More recently, diameter $\geq 3 \mathrm{~cm}$ and the presence of multiple tumours were confirmed as significant predictors of NMIBC progression ${ }^{123}$.

\subsection{Early cystoscopic findings}

In a study by Sylvester et al, persistent disease diagnosed at the first surveillance cystoscopy after induction intravesical treatment was a risk factor associated with progression, which occurred in $25.3 \%$ versus $8.7 \%$ of patients with or without pathologically confirmed malignancy, respectively ${ }^{83}$. Early RCTs in the 1980's by the EORTC group showed that the number of tumours, as well as a recurrence rate higher than 1 per year, were associated with future intravesical recurrence (LE 2) ${ }^{124}$. Later, secondary analyses of RCTs also confirmed the 
association of previous recurrence rates (more than 1 per year) and multifocality with early tumour recurrence (or persistent disease) at the first surveillance cystoscopy after treatment, potentially reflecting not only small, missed tumours at first TURBT, but also resistance to first line intravesical therapy ${ }^{118,125,126}$.

\subsection{Histology}

Approximately $90 \%$ of bladder cancers are pathologically classified as UCs (previously named "transitional cell carcinomas"), arising from the bladder urothelium. Pure squamous cell carcinoma and pure adenocarcinoma are less commonly diagnosed (2-5\% and 2\% of cases, respectively $)^{127}$. Among UC's, $75 \%$ are classified as pure, while $25 \%$ harbour a secondary histological variant, the most common being squamous (20-40\%) and glandular differentiation $(18 \%)^{127}$. Particularly for NMIBC, variant histologies have been under-reported by pathologists in up to $44 \%$ of cases $^{128}$. Furthermore, initial diagnosis through biopsies or TURBT specimens have shown low sensitivity (35\% and $43 \%$, respectively) to predict variant histologies after RC according to retrospective data ${ }^{129}$. Variant histologies have implications for management and prognosis, due to their higher risk for under-staging, higher risk of progression, and possibly differential response to treatment. Therefore, a pathological review, preferably by a dedicated uro-pathologist, should be considered in settings where variant histology is suspected by the pathologist or atypical tumours are seen by the urologist during TURBT (e.g., sessile mass) (LE 3 ; weak recommendation $)^{129}$.

Not all variant histologies have the same prognostic implications. For example, controlling for stage, oncological outcomes are similar between pure UC versus UC with squamous or glandular differentiation ${ }^{130,131}$. Therefore, NMIBC with squamous or glandular differentiation can be managed similarly to pure UC (LE 3, weak recommendation). The presence of nested variant of UC has been associated with higher stage and nodal invasion, but main oncological outcomes were comparable to patients with pure UC when matched by stage in both the NMIBC and MIBC setting ${ }^{132,133}$. Other variant histologies, such as micropapillary, plasmacytoid and sarcomatoid differentiation are associated with higher rates of progression and worse prognosis, prompting consideration for aggressive treatment with upfront RC in the majority of cases (LE 3) ${ }^{127,134,135}$. Small cell (or neuroendocrine) bladder cancer is rarely diagnosed at an early stage and requires individualized approach with multi-disciplinary tumour board review. Neoadjuvant chemotherapy followed by consolidation with either RC or radiationbased therapy should be considered ${ }^{135}$.

\section{Risk stratification}

- All patients with NMIBC should be stratified according to the risk of both recurrence and progression for adequate patient counselling and treatment 


\section{planning ( $L E$ 2; strong recommendation). The modified CUA risk stratification system is a suitable tool for this purpose.}

After diagnosis, clinical and pathological factors must be considered to allow for risk stratification of NMIBC. In order to estimate risks for recurrence and progression and to plan additional treatments after TURBT, the EORTC developed a comprehensive scoring system based on 2,596 patients from 7 clinical trials ${ }^{83}$. A score is calculated to estimate the risks of recurrence and progression at 1 and 5 years, based on 6 prognostic factors: number of tumours (single versus $2-7$, versus $\geq 8$ ), tumour size $(<3 \mathrm{~cm}$ versus $\geq 3 \mathrm{~cm}$ ), number of previous recurrences $(\leq 1$ per year versus $>1$ per year), $\mathrm{pT}$ stage (pTa versus pT1), presence of concurrent CIS and grade (available at http://www.eortc.be/tools/bladdercalculator//) ${ }^{83}$.

The EORTC risk calculator, however, likely overestimates recurrence (15-61\%) and progression (1-17\%) at 1 year when compared to high-risk NMIBC patients receiving BCG, since the majority of the patients in the included RCTs were treated with intravesical chemotherapy, which is associated with inferior outcomes compared to BCG. Additionally, all 171 patients treated with BCG in this analysis underwent only an induction course, which is today considered suboptimal for high-risk patients ${ }^{83}$. To address that, the CUETO developed a similar calculator, also to predict recurrence and progression, but including 1,062 patients treated with induction (6 weekly instillations for 6 weeks) and maintenance (6 two-weekly doses for up to 12 doses) of $\mathrm{BCG}^{122}$.

A recent multicentre study by Sylvester et al using individual patient data for 3,401 NMIBC patients sought to stratify patients into risk groups for progression, incorporating the grade categories of the WHO 2004/2016 grading system, since it was not considered in previous systems by the EORTC ${ }^{123}$. In a multivariable analysis, age $>70$ years was significantly associated with disease progression (HR 1.72, 95\% CI 1.24-2.40; $\mathrm{p}=0.001$ ), together with T1 stage (HR 2.20, 95\% 1.53 - 3.16; p<0.001), high grade disease (HR 2.33, 95\% CI 1.58 - 3.42), multiple tumours (HR 1.64, 95\% CI $1.17-2.29$ ), tumour diameter $\geq 3 \mathrm{~cm}$ (HR 1.97, 95\% CI 1.41 -2.77 ) and concomitant CIS (HR 2.76, 95\% CI $1.62-4.70)^{123}$. As a result, age $>70$ years was included as an additional factor to be considered when stratifying patients based on progression risk (LE 2; strong recommendation). Importantly, patients with initial recurrent disease, primary CIS with no associated papillary tumours, CIS in the prostatic urethra, LVI, variant histologies and those treated with intravesical BCG were not included in the analysis, as these factors could potentially affect progression rates ${ }^{123}$.

The modified CUA risk stratification method is proposed here (Table 3). Although validation in larger populations is required, this classification can be easily implemented in daily practice. It stratifies patients individually in low-, intermediate- and high-risk NMIBC. 


\begin{tabular}{|c|c|}
\hline Risk group & Tumour characteristics \\
\hline Low & $\begin{array}{l}\text { 1. PUNLMP } \\
\text { 2. Primary, solitary, and small }(<3 \mathrm{~cm}) \text { LG Ta }\end{array}$ \\
\hline Intermediate & $\begin{array}{l}\text { Patients without CIS who are not included in the other risk categories: } \\
\text { 1. Recurrent, multifocal, and/or large }(>3 \mathrm{~cm}) \mathrm{LG} \mathrm{Ta} \\
\text { - Consider substratification: } \\
\text { a) Low-intermediate-risk: } 0 \text { factors* - consider treating as low-risk patients } \\
\text { b) Intermediate-risk: } 1-2 \text { factors } \\
\text { c) High-intermediate-risk: } \geq 3 \text { factors - consider treating as high-risk patients } \\
\text { *Multiple tumours, } \geq 3 \mathrm{~cm} \text {, time to recurrence ( }<1 \text { year), and frequency of } \\
\text { recurrence ( }>1 \text { / year) } \\
\text { 2. Primary, solitary, and small }(<3 \mathrm{~cm}) \mathrm{HG} \mathrm{Ta} \\
\text { - Consider treating as high-risk patients }\end{array}$ \\
\hline High & $\begin{array}{l}\text { Any: } \\
\text { 1. } \mathrm{T}^{\dagger} \\
\text { 2. Recurrent, or multiple, or } \geq 3 \mathrm{~cm} \text { HG Ta } \\
\text { 3. Presence of CIS (primary or concomitant) } \\
\text { †Very high-risk: } \\
\text { HG T1 with any of the following: } \\
\text { a) Multiple and } \geq 3 \mathrm{~cm} \\
\text { b) Presence of concurrent CIS (in the bladder or prostatic urethra) } \\
\text { c) Presence of LVI } \\
\text { d) Variant histology (e.g., micropapillary, plasmacytoid, sarcomatoid, } \\
\end{array}$ \\
\hline
\end{tabular}

${ }^{\S}$ Modified from EORTC and CUETO stratification tools, Sylvester et al ${ }^{123}$, and other major guidelines. CIS: carcinoma in situ; HG: high-grade; LG: low-grade; LVI: lymphovascular invasion; PUNLMP: papillary urothelial neoplasm of low malignant potential.

\section{Transurethral resection of bladder tumour (TURBT)}

- Patients presenting with a bladder tumour should undergo initial TURBT for diagnostic confirmation and pathological evaluation ( $L E 2$; strong recommendation).

- Initial TURBT aims for complete tumour resection with sampling of the underlying detrusor muscle as the first step of curative-intent treatment of NMIBC (LE 2; strong recommendation). Patients with presumed LG Ta or CIS might be spared from muscle sampling at initial TURBT ( $L E 3$; weak recommendation).

Initial TURBT is a key step in the management of bladder cancer. It has a diagnostic, prognostic, and therapeutic role, and its quality can impact oncological outcomes. Sampling of 
detrusor muscle is an important quality indicator (in tumours other than PUNLMP, LG Ta, and CIS), and its absence is associated with risk of under-staging, residual disease, and recurrence ${ }^{136}$.

Steps for a standardized TURBT are presented here (Table 4)137-139. Systematic characterization and mapping of all lesions should be performed. All visible tumours should be completely resected, and ideal depth should include the detrusor muscle (muscularis propria) to allow for optimal staging. Multiple tumours should be sent as separate specimens. For large tumours, consider sending the tumour base and deep resection separately. Bimanual examination should be performed.

Anesthetic technique, whether general with neuromuscular blockade (associated with reduction of the obturator reflex and lower risk of bladder perforation) ${ }^{140}$, or spinal anesthesia should be recommended based on patient's risk factors, comorbidities, and tumour location ${ }^{141}$. Bipolar energy was shown to reduce the obturator nerve reflex and rates of bladder perforation when compared to resection with monopolar energy, according to a meta-analysis on 8 studies (6 RCTs $)^{142}$. Another meta-analysis of 8 RCTs with 1,147 patients reinforced the association of bipolar resection and shorter hospital stay, resection time and lower rates of bladder perforation and hemoglobin fall, although no difference was seen with respect to the obturator reflex when compared to monopolar resection ${ }^{143}$.

\section{Table 4. Checklist for high-quality TURBT}

\section{Stepwise checklist for high quality TURBT}

NMIBC objective: provide enough information for adequate risk stratification

Cystoscopy:

1. Provide detailed description of urethra, bladder walls, and lesions (number, size, appearance, suspicion for concurrent / primary CIS);

2. Report visual impression of clinical stage and grade;

3. Collect washing or voided urine cytology if not previously obtained.

TURBT:

4. Completely resect all visible tumours and suspicious areas;

5. Send labeled tumour specimens separately;

6. Avoid excessive fulguration;

7. Use enhanced visualization techniques when available;

8. Use bipolar energy when indicated and available;

9. Deep resection of the detrusor muscle - send deep specimens separately.

10. Random biopsies (bladder and prostatic urethra) if indicated.

11. Ensure adequate hemostasis.

12. Assess bladder wall integrity after resection (evaluate for perforation).

13. Perform bimanual exam under anesthesia.

CIS: carcinoma in situ; NMIBC: non-muscle-invasive bladder cancer; TURBT: transurethral resection of bladder tumour. 


\subsection{TURBT optimization}

- When available, blue light cystoscopy (BLC) ( $L E$ 1; weak recommendation) or narrow band imaging (NBI) (LE 2; weak recommendation) can increase tumour detection at first TURBT and reduce recurrence risk.

Several clinical trials have evaluated enhanced imaging methods such as BLC and NBI with the intent of improving tumour detection rates and reducing disease recurrence. BLC and NBI are potential useful tools in the initial management and surveillance of NMIBC.

\subsubsection{Blue light cystoscopy}

Fluorescent cystoscopy or BLC uses a photosensitizing agent, such as 5-aminolevulinic acid (ALA) or hexyl aminolevulinic acid (HAL). Instilled in the bladder before the procedure for 1-4 hours ${ }^{144}$, these substances are metabolized to protoporphyrin IX by tumour cells, showing a red fluorescence under blue light (380-470nm) which provides contrast between tumours and the normal urothelium ${ }^{145}$. Currently, the only available agent is HAL.

Clinical trials have shown a consistent positive impact of BLC on NMIBC recurrence ${ }^{146-}$ ${ }^{149}$. A large meta-analysis of 2,212 patients with primary and recurrent NMIBC from 9 prospective studies showed that adding BLC-HAL to WLC increased detection rates by $14.7 \%$ for Ta tumours and by $40.8 \%$ for CIS lesions (LE 1$)^{150}$. Furthermore, at least one additional lesion was detected in $24.9 \%$ of patients and there was a significant reduction in recurrence rates at 12 months for all risk subgroups, including patients treated with BCG (LE 1) ${ }^{150}$. The recurrence benefit of BLC-HAL was also confirmed in studies with longer followup ${ }^{151}$. Geavlete et al showed 2-year recurrence rates of $31.2 \%$ versus $45.6 \%(\mathrm{p}=0.001)$, while Gallagher et al showed 3-year recurrence rates of $39.0 \%$ versus $53.3 \%(\mathrm{p}=0.02)$ for BLC-HAL versus WLC, respectively ${ }^{152,153}$. Despite a trend for BLC in decreasing progression rates and prolong time to progression, current data is inconclusive, and its impact on oncologic outcomes other than recurrence remains unclear ${ }^{154}$.

Most clinical trials testing BLC have not used single dose chemotherapy at the time of TURBT, so the impact of combining both interventions remains uncertain. All patients in the trial by Geavlete et al received single dose chemotherapy ${ }^{152}$. However, in another prospective randomized trial by O'Brien et al, there was no significant difference in recurrence rates between WLC and BLC in 249 patients undergoing TURBT with single dose mitomycin-C (MMC) ${ }^{155}$. The lack of difference could be explained by an ablative effect of MMC on potentially missed lesions at $\mathrm{WLC}^{155}$.

Especially when proposing new technologies associated with bladder cancer care, costeffectiveness needs to be evaluated, as bladder cancer has the highest lifetime treatment cost per patient among all cancers ${ }^{156,157}$. Regarding implementation of BLC at initial TURBT, a costeffectiveness analysis was performed and found that BLC-HAL at initial TURBT was associated 
with better quality-adjusted life-years and overall cost over time than WLC, by reducing NMIBC recurrences ${ }^{158}$. Meanwhile a study by Klaassen et al suggested that, despite high initial costs associated with the implementation of BLC, this approach could result in 87 to 338 fewer bladder recurrences annually in Canada ${ }^{159}$.

\subsubsection{Narrow-band imaging}

NBI enhances visualization of bladder tumours by using filters to narrow the bandwidth of light into wavelengths of $415 \mathrm{~nm}$ (blue) and 540nm (green). Since these wavelengths correspond to the peak absorption of hemoglobin, NBI provides enhanced contrast between benign and hypervascular malignant lesions ${ }^{160}$. NBI is activated by pressing a switch in cystoscopes equipped with this technology and does not require bladder instillation of any kind before the procedure.

Several articles compared NBI with WLC, focusing on recurrence rate reduction ${ }^{161,162}$. In 2012, Naselli et al randomized 223 patients to receive TUR with NBI or WLC. With a median followup of 11 months, recurrence rates were found to be $3.9 \%$ versus $16.7 \%(\mathrm{p}=0.008)$ at 3 months and $31.6 \%$ versus $51.4 \%(\mathrm{p}=0.014)$ at 1 year for NBI versus WLC, respectively ${ }^{163}$. Another RCT by Geavlete et al showed a higher specificity for detecting CIS using NBI in comparison with WLC (53.8\% versus $15.4 \%$, respectively $)^{164}$. Finally, a multicentre trial by the Clinical Research Office of the Endourological Society (CROES) randomized 965 patients to undergo NBI versus WLC-assisted TURBT. Their results showed similar overall recurrence rates of $27.1 \%$ for WLC versus $25.4 \%$ for NBI at 1 year $(\mathrm{p}=0.585)$, although subgroup analysis showed that the 1-year recurrence rate for low-risk patients was significantly lower for NBI over WLC $(5.6 \%$ versus $27.3 \%$, respectively $-\mathrm{p}=0.002)(\operatorname{LE} 2)^{165}$. The authors stated that the effectiveness of NBI for low-risk patients can be explained by the fact that most recurrences might be caused by small, overlooked lesions at WLC that were identified through NBI. Finally, in a meta-analysis, Kang et al compared 1,084 patients from 6 studies (out of which 4 were RCT's) and found lower recurrence-free survival rates at 3 months (RR 0.39, $\mathrm{p}<0.0001)$ ), 1 year (RR 0.52, $\mathrm{p}<0.00001)$ and 2 years (RR 0.60, $\mathrm{p}=0.004$ ) for NBI when compared to WLC ${ }^{166}$.

\subsubsection{En-bloc TURBT}

En-bloc resection aims for complete removal of the bladder tumour with the adjacent bladder tissue and muscularis propria in one single specimen, being first described in the late 1990 's ${ }^{167,168}$. Based on historical oncological principles for other malignancies, it claims to be a "no touch" technique and is advocated by some urologists under the rationale that it provides for better quality specimens (less fulguration), improved accuracy of pathology diagnosis, reduced number of floating tumour cells and lower risk of bladder perforation during TURBT ${ }^{169}$. In a randomized study by Hashem et al, the detrusor muscle was present in $98 \%$ of specimens of patients undergoing holmium laser en-bloc resection versus $62 \%$ undergoing conventional 
TURBT $(\mathrm{p}<0.001)^{170}$. On the contrary, the multicentric randomized study by Gakis et al showed no statistical difference regarding the presence of detrusor muscle in en-bloc versus conventional TURBT specimens ( $77.4 \%$ versus $66.7 \%$, respectively; $\mathrm{p}=0.28$ ), although a higher proportion of negative surgical margins was observed with en-bloc technique (57\% versus $9 \%$, respectively; $\mathrm{p}<0.001)^{171}$. Regarding $\mathrm{T} 1$ sub-staging, the concordance among pathologists when assessing depth of T1 invasion is higher when specimens are obtained by en-bloc TURBT, in comparison to conventional TURBT ${ }^{172}$.

A large systematic review with meta-analysis by Teoh et al included 32 studies for qualitative and 10 RCT's for quantitative analysis on the effectiveness of en-bloc resection versus standard TURBT ${ }^{173}$. According to this study, en-bloc resection was associated with longer operative time (mean difference $9.07 \mathrm{~min}, 95 \% \mathrm{CI} 3.36-14.8, \mathrm{p}=0.002$ ), shorter post-operative irrigation time (mean difference $-7.2 \mathrm{~h}, 95 \% \mathrm{CI}-9.3$ to $-5.2, \mathrm{p}<0.001$ ) and lower rate of bladder perforation (RR 0.30, 95\% CI $0.11-0.83, \mathrm{p}=0.02)$ when compared to TURBT. On the other hand, there was no statistical difference regarding the presence of muscularis propria in the final specimen nor recurrence rates at 1,2 and 3 years ${ }^{173}$. Larger randomized studies are needed to define the role of en-bloc TURBT in clinical practice, including its learning curve, impact in recurrence rates and whether it could potentially spare a subset of patients from restaging TURBT or additional adjuvant instillations ${ }^{174}$. In conclusion, en-bloc TURBT is considered an emerging resection technique that may improve detrusor sampling and provide for better quality specimens for pathological evaluation (LE 3; weak recommendation). Its benefit on disease recurrence has not been established and further studies are needed prior to recommending its routine use.

\subsection{Restaging TURBT}

- A restaging TURBT should be performed in patients with T1 NMIBC, or when a complete resection was not achieved with the first TURBT (LE 2; strong recommendation). Restaging TURBT is not required in patients who will proceed to RC based on the findings of the first TURBT.

- In select cases of HG Ta tumours (e.g., large and/or multiple tumours), a re-staging TURBT might be considered ( $L E 3$; weak recommendation).

- The suggested window for a restaging TURBT is within 6 weeks of the first resection (LE 3; weak recommendation).

Restaging TURBT (re-TURBT) changes NMIBC management in many patients ${ }^{175}$. It not only improves staging but also has therapeutic benefit and should be performed in all patients with T1 disease, aiming for the identification of occult muscle-invasive disease and resection of initially unresected lesions ${ }^{125}$. 
In 1998, Herr published a series of 150 patients who underwent a re-TURBT for newly diagnosed and recurrent bladder cancer, showing that 76\% (114) had residual tumour at reTURBT, while $29 \%$ of those with NMIBC at initial TURBT were upstaged to MIBC $(\geq \mathrm{T} 2)$ after a second-look resection ${ }^{175}$. Other studies reported up to $9.5 \%$ upstaging for HG Ta tumours (to $\geq$ $\mathrm{T} 1$ ) and $28 \%$ for $\mathrm{T} 1$ tumours (to $\geq \mathrm{T} 2)^{176,177}$. Divrik et al analyzed 210 patients with newly diagnosed T1 who were randomized to undergo re-TURBT or not, and were followed until death or a minimum time of 54 months ${ }^{178}$. They reported 5 -year RFS of $59 \%$ vs $32 \%(\mathrm{p}=0.0001)$ and progression-free survival (PFS) of 93\% versus 79\% ( $\mathrm{p}=0.0001$ ) favouring re-TURBT. Additionally, re-TURBT was an independent predictor for both lower recurrence and progression rates in multivariate analysis (LE 2) ${ }^{178}$. This study, however, likely overestimated the effect of re-TURBT on long-term recurrence and progression and was criticized by lack of pre-defined end points, sample size power, method of randomization, and potential impacts of further intravesical therapy on oncological outcomes of both arms ${ }^{178}$.

Cumberbatch et al recently published a systematic review including 31 studies (only 1 RCT) and 8,409 patients with HG tumours ${ }^{179}$. Re-TURBT resulted in upstaging rates of $0.4 \%$ [0$8 \%$ ] and $8 \%$ [0-32\%] of initial pTa versus initial pT1 tumours, respectively (LE 3). In pTa patients, a re-TURBT was associated with lower rates of recurrence, but not progression, while for pT1 patients, it resulted in lower rates of progression and overall mortality, with additional trend for lower cancer-specific mortality. The majority of residual lesions was identified in the original tumour resection bed ${ }^{179}$. Another meta-analysis on 29 studies confirmed the presence of residual disease in $56 \%$ of $\mathrm{pT} 1$ patients, with $10 \%$ of patients experiencing upstaging to $\mathrm{pT} 2$ at re-TURBT ${ }^{180}$.

Smaller studies have focused on the value of re-TURBT in patients who subsequently receive BCG therapy. A retrospective study on 347 patients presenting with HG Ta or T1 tumours, who were treated with 6-weekly induction course of BCG, demonstrated a risk of recurrence at first followup evaluation in 57\% of patients who did not undergo re-TURBT before BCG versus $29 \%$ when a re-TURBT was performed $(\mathrm{p}=0.001)^{181}$. The rate of progression in this study was reduced from $34 \%$ without TURBT to $7 \%\left(\mathrm{p}=0.001\right.$ ) with re-TURBT ${ }^{181}$. Another retrospective study with 427 patients with a median followup of 63 months showed that patients with high-risk NMIBC benefited from induction and maintenance BCG even when re-TURBT confirmed absence of any tumour (pT0) ${ }^{182}$. These results suggest that a complete resection before BCG therapy is important for obtaining optimal results from this approach (LE 3; weak recommendation).

Timing of re-TURBT is also important. A multicentre retrospective study was performed on 242 patients with high-risk NMIBC and treated with induction and at least 1 year of maintenance $\mathrm{BCG}^{183}$. With a followup of 29.4 months, multivariable analysis showed that a reTURBT beyond 42 days was an adverse independent predictor for both recurrence (OR 3.60, $\mathrm{p}=$ 
$0.001)$ and progression $(\mathrm{OR} 2.14, \mathrm{p}=0.003)^{183}$. Another retrospective analysis on 491 patients with high-risk NMIBC treated with BCG therapy found no recurrence or progression benefit for patients restaged beyond 8 weeks and suggested the optimal window for re-TURBT would be between 2-6 weeks ${ }^{184}$. Therefore, a restaging TURBT should be performed within 6 weeks of the first resection (LE 3; weak recommendation).

The presence of benign muscularis propria confirmed in the first TURBT has been identified as the most significant predictor of having no tumour (pT0) at restaging TURBT (OR 3.05, $\mathrm{p}=0.03)(\operatorname{LE} 3)^{185}$. More recent studies reinforced the indication of a re-TURBT when detrusor muscle is not reported at the first resection and questioned the role of a repeat TURBT in patients with single and small $\mathrm{T} 1$ or HG Ta disease with benign muscularis propria at first resection ${ }^{186-189}$. For T1 tumours, Cumberbatch et al showed that when the detrusor muscle was absent or not reported in the first resection, a restaging TURBT resulted in upstaging to MIBC in up to $45 \%$ of the cases ${ }^{179}$. Although the absence of muscularis propria in the first TURBT specimen is a clear indication for a restaging TURBT in T1 tumours, a similar benefit for Ta tumours is less clear. Therefore, in select cases of HG Ta (e.g., multiple and / or $\geq 3 \mathrm{~cm}$ tumours), a restaging TURBT might be considered (LE 3; weak recommendation) ${ }^{190,191}$.

\subsection{Random bladder biopsies}

- Patients presenting with a positive urine cytology, but normal appearing bladder at WLC and normal upper urinary tract imaging are at higher risk of harbouring occult CIS and should undergo random bladder biopsies (or use of BLC with directed biopsies) (LE 2; strong recommendation).

A secondary analysis of two EORTC trials including low-risk (EORTC 30863) versus intermediate and high-risk (EORCTC 30911) patients showed little benefit of routine random bladder biopsies in detecting CIS (LE 2) ${ }^{192}$. In this study, $90 \%$ of patients had negative random biopsies, although concurrent CIS was found in $1.5 \%$ versus $3.5 \%$ of patients with low versus intermediate/high-risk NMIBC, respectively ${ }^{192}$. In another study by Hara et al including 173 patients, up to $59 \%$ of high-risk patients with normal-appearing cystoscopy had confirmed CIS in biopsy specimen and a significant association between a positive urine cytology and detection of CIS through random bladder biopsies was demonstrated ${ }^{193}$. Urine cytology had a sensitivity of $87.1 \%$ and specificity of $63 \%$ for detecting CIS in a recent meta-analysis by Subiella et al (LE 2), meaning that $37 \%$ of patients with negative urine cytology samples might in fact harbour CIS $^{96}$. In this same study, the overall detection rate of CIS through random bladder biopsies of normal appearing mucosa was $17.4 \%$, which increased to $57.3 \%$ when urine cytology was positive ${ }^{96}$. In a setting of normal cystoscopy and normal upper urinary tract imaging, random bladder biopsies should be performed if urine cytology is positive (LE 2; strong recommendation). In this scenario, enhanced imaging methods may play a role in identifying 
targets for directed biopsy when cystoscopy is normal, also aiming for higher detection of CIS in those cases (LE 3; strong recommendation) ${ }^{158}$.

\subsection{Prostatic urethral involvement (PUI)}

- Biopsies or transurethral resection of the prostatic urethra should be included with random bladder biopsies in the presence of a positive urine cytology, but normal appearing bladder at WLC and normal upper urinary tract imaging ( $L E 3$; strong recommendation).

- Prostatic urethral biopsy (or transurethral resection) can also be considered in the presence of extensive bladder CIS or tumour at the bladder neck or trigone ( $L E 3$; weak recommendation).

- Patients with PUI with CIS restricted to the urethral mucosa can be managed conservatively with transurethral resection of prostate (TURP) plus intravesical BCG ( $L E$ 3; weak recommendation). Repeat prostatic urethral biopsies after induction BCG should be considered ( $L E 3$; weak recommendation). RC can be discussed as an alternative option ( $L E 4$; weak recommendation).

- In patients with HG T1 or CIS extending into the prostatic ducts, RC should be considered ( $L E$ 3; weak recommendation). TURP followed by intravesical BCG is an alternative option. In this instance, close followup with repeat prostatic urethral biopsies after induction BCG should be considered ( $L E 3$; weak recommendation).

- In patients with prostatic stromal invasion, neoadjuvant cisplatin-based chemotherapy followed by $\mathbf{R C}$ is recommended ( $L E$ 3; strong recommendation - refer to MIBC guideline).

Incidence of primary prostatic urethral UC is rare (1-4\%), but contiguous PUI by bladder cancer can range from $12-48 \%$ according to retrospective series (LE 3) ${ }^{194,195}$. The prostatic urethra is less exposed to intravesical therapy, allowing CIS and high-risk papillary disease to advance through the prostatic ducts into the prostatic stroma ${ }^{196}$. A clear association between CIS detected in the prostatic urethra and worse oncological outcomes has been demonstrated ${ }^{197,198}$. Palou et al retrospectively analyzed 146 NMIBC patients with HG T1 tumours treated with TURBT followed by BCG and showed that CIS was present in the prostatic urethra of $11.7 \%$ of the cases and associated with worse rates of recurrence (HR 2.53, $\mathrm{p}=0.0003)$, progression (HR 3.59, $\mathrm{p}=0.001)$ and disease-specific mortality $(\mathrm{HR} 3.53, \mathrm{p}=0.004)^{199}$.

A retrospective study by Mungan et al including 340 patients treated with TURBT for primary NMIBC showed that the only significant predictive factor for PUI was the presence of multiple tumours in multivariable analysis (LE 3$)^{200}$. Similarly, a study by Brant et al evaluated 177 patients with NMIBC who underwent RC, and had no upstaging in final pathology ${ }^{201}$. It revealed that PUI was significantly associated with worse RFS $(p=0.01)$, DSS $(p=0.03)$ and 
overall survival (OS) $(\mathrm{p}<0.01)$ on log-rank test. In a logistic regression model, significant predictors for having PUI in final RC specimen were previous intravesical therapy (OR 2.90, $\mathrm{p}<0.02$ ), positive urethral / ureteral margins ( $\mathrm{OR} 4.01, \mathrm{p}=0.01)$ and multifocal tumours (OR 7.56, $\mathrm{p}<0.001$ ) (LE 3), while PUI was an independent predictor of overall mortality in multivariable analysis (HR 2.08, $\mathrm{p}<0.01)^{201}$.

The depth of invasion of PUI is associated with oncological outcomes. Solsona et al evaluated 96 patients with PUI and classified tumours in 3 groups: limited to the prostatic urethral mucosa (group 1), prostatic ductal invasion (group 2) and prostatic stromal invasion (group 3$)^{202}$. They found a statistically significant better prognosis for groups 1 and 2 when compared to group 3 ( $\mathrm{p}<0.001$ ) (LE 3). Multivariable analysis showed that stromal (but not mucosal or ductal) invasion, bladder CIS and pan-urothelial involvement were independent predictors for worse $\mathrm{OS}^{202}$. The prognostic association of the depth of PUI was later reinforced by other series, where stromal involvement was associated with 5-year OS of 40-50\% versus $100 \%$ for PUI restricted to the urethral mucosa ${ }^{195}$.

Prostatic urethral biopsies can be considered in patients with tumours located at the bladder neck and trigone, those with associated bladder CIS and whenever bladder urine cytology is positive in the setting of a negative cystoscopy and normal upper tract imaging (LE 3 ; weak recommendation ${ }^{203}$. Moreover, for highest accuracy, any suspicious areas in the prostatic urethra should be biopsied along with 5 and 7 o'clock (precollicular area) at the level of the verumontanum where the highest concentration of prostatic ducts is located (LE 3; weak recommendation $)^{203}$.

Patients with PUI limited to the mucosa have better prognosis and can be managed with transurethral resection of the prostate (TURP), followed by intravesical $\mathrm{BCG}^{199,204}$. TURP aims for accurate staging, surgical resection of the disease, and bladder neck opening, which allows for better exposure of the prostatic urethra to BCG instillations ${ }^{205}$. On the other hand, RC should be considered in patients with ductal invasion (LE 3; weak recommendation), although bladder preservation with TURP followed by BCG has been described in small retrospective series ${ }^{206}$. If managed conservatively, PUI with associated CIS needs close followup with early assessment after BCG induction therapy, including early prostatic urethral re-biopsy, due to higher risk of local recurrences and distant metastasis (LE 3; weak recommendation) ${ }^{207}$. Finally, invasion of the prostatic stroma requires aggressive treatment with neoadjuvant chemotherapy followed by $\mathrm{RC}+/$ - urethrectomy (refer to MIBC guideline) ${ }^{204}$ =

\section{Intravesical therapy}

First-line adjuvant intravesical therapy options consist mainly of chemotherapy and immunotherapy (including BCG). It is administered with therapeutic (treatment of CIS or residual non-visible tumour) and prophylactic (prevention of recurrence and progression of disease) intents. 


\subsection{Intravesical chemotherapy}

\subsubsection{Single instillation of chemotherapy (SIC) post-TURBT}

- SIC (with MMC, epirubicin, doxorubicin, pirarubicin or gemcitabine) should be offered to all patients with presumed low-risk NMIBC at TURBT and should be administered within $\mathbf{2 4}$ hours after endoscopic resection (LE 1; strong recommendation).

- SIC is recommended for intermediate-risk NMIBC and for patients with $\leq 1$ recurrence/year and EORTC recurrence score < 5 ( $L E$ 1; strong recommendation). SIC should be discussed even when further adjuvant intravesical chemotherapy is planned ( $L E 2$; weak recommendation).

- The benefit of SIC in patients with high-risk NMIBC is unclear when BCG is planned as adjuvant treatment ( $L E 3$ ).

- SIC should not be administered after extensive resection or when bladder perforation is suspected ( $L E 3$; strong recommendation).

Injury to the bladder wall and fulguration during TURBT can facilitate tumour cell reimplantation. When TURBT is performed alone without SIC, persistent NMIBC at first 3month cystoscopy can be as high as $21 \%^{208}$. SIC was initially proposed with the intent of reducing the number of floating malignant cells in the bladder after a TURBT, preventing cancer cell reimplantation and reducing early recurrence rates after resection. In addition, this approach might have an ablative effect on small occult tumours ${ }^{209}$.

Several RCTs have identified a role of SIC after TURBT in reducing NMIBC recurrence. At least 5 RCT's performed from 1984 to 2011 showed a relative risk reduction in early recurrence rates of about $50 \%$ with a SIC with $\mathrm{MMC}^{210-214}$. Epirubicin was also associated with a decrease in recurrence risk of up to $50 \%$ in 2 years $^{215-218}$. Additionally, comparable results were reported with thiothepa, doxorubicin and pirarubicin ${ }^{219-221}$. More recently, gemcitabine was tested in an RCT by Messing et $\mathrm{al}^{222}$. In comparison with saline irrigation, SIC with gemcitabine given within 3 hours of TURBT was associated with a 12\% absolute risk reduction in 4-year recurrence rates, conferring a relative risk reduction of 34\% (HR 0.66; 95\% CI $0.48-0.90$; $\mathrm{p}<$ $0.01)^{222}$. Intravesical gemcitabine was associated with minimal adverse effects and low $\operatorname{cost}^{222}$. The most utilized protocols for SIC are presented in Table 5. 


\begin{tabular}{|l|l|l|}
\hline \multicolumn{3}{|l|}{ Table 5. Regimens of single instillation of chemotherapy } \\
\hline Chemotherapy & Dose & Protocol \\
\hline Gemcitabine & $2 \mathrm{~g}$ & Dilution in $100 \mathrm{ml}$ of saline $(60 \mathrm{~min})$ \\
\hline Mitomycin-C & $40 \mathrm{mg}$ & Dilution in $20-40 \mathrm{ml}$ of water $(60 \mathrm{~min})$ \\
\hline Doxorubicin & $30 \mathrm{mg}$ & Dilution in $30 \mathrm{ml}$ of saline $(60 \mathrm{~min})$ \\
\hline Epirubicin & $50-80 \mathrm{mg}$ & Dilution in $50 \mathrm{ml}$ of saline $(60 \mathrm{~min})$ \\
\hline Pirarubicin & $30 \mathrm{mg}$ & Dilution in $30 \mathrm{ml}(60 \mathrm{~min})$ \\
\hline
\end{tabular}

For intermediate and high-risk patients, however, results are controversial and heterogeneous regarding different drugs and protocols ${ }^{223}$. In 2004, Sylvester et al published a meta-analysis including 7 RCTs and 1,476 patients, mostly low-risk, where 1 immediate instillation of chemotherapy with either MMC, epirubicin or pirarubicin after TURBT was associated with recurrence rate of $36.7 \%$, versus $48.4 \%$ for the TURBT alone, resulting in a $39 \%$ decrease in the odds of recurrence favouring SIC (LE 1; strong recommendation). The benefit, however, was not statistically significant for patients with multiple tumours when adjusted for stage $^{224}$. With similar results, two additional meta-analyses endorsed the use of immediate intravesical instillation after a TURBT, although highlighting concerns on heterogeneity and publication bias among trials ${ }^{225,226}$.

The most recent meta-analysis revisiting the topic in 2016 was published again by Sylvester et al, this time including individual data analysis of 2,278 randomized patients ${ }^{227}$. A reduction of $35 \%$ in the relative risk of recurrence was reported favouring SIC (HR 0.65, $\mathrm{p}<0.001$ ), with 5-year recurrence of $44.8 \%$ versus $58.8 \%$ for TURBT alone (LE 1 ). This study showed no benefit of SIC for patients with more than one recurrence per year and those with an EORTC recurrence score $\geq 5$. Interestingly, OS was significantly lower in patients meeting these higher risk criteria when treated with SIC. The authors stated that this was possibly influenced by the number of high-risk patients with intrinsic poor performance status and prognosis who would ultimately not be the ideal candidates for $\mathrm{SIC}^{227}$.

A large multicentre trial by Bosschieter et al randomized 2,243 patients to receive intravesical instillation of MMC within the first 24 hours after TURBT (immediate) or 2 weeks after the procedure (delayed), followed in both scenarios by adjuvant instillations of chemotherapy for intermediate ( 9 doses) and high-risk (15 doses) patients ${ }^{228}$. Recurrence rates at 3 years were of $27 \%$ in the immediate versus $36 \%$ in the delayed instillation group $(p<0.001)$, reflecting a $34 \%$ reduction in the relative risk favouring immediate instillation (LE 1). Despite possible selection bias and unique risk stratification (primary and solitary LG Ta and LG T1 tumours were classified as low-risk; multiple tumours were classified as high-risk), this was the first study suggesting lower rates of recurrence even in patients with intermediate and high-risk disease who received further adjuvant intravesical instillations ${ }^{228}$. Moreover, lower recurrence 
rates were reported for intermediate and high-risk patients when compared to low-risk, possibly as a result of further adjuvant intravesical chemotherapy administered in these patients ${ }^{228}$. Later, a reanalysis of patients included in this trial was conducted ${ }^{229}$. Using updated risk definition, the authors concluded that the benefit of SIC was significant regardless of risk group and suggested that SIC should not be withheld from intermediate and high-risk patients ${ }^{22}$.

Discussion regarding conflicting data taken from the largest meta-analysis and the largest RCT conducted so far were published ${ }^{230-232}$, recommending SIC for all low-risk patients (LE 1; strong recommendation) and intermediate-risk patients regardless of whether further adjuvant intravesical chemotherapy is given (LE 2; weak recommendation). Patients who fall in the highrisk category but meet the criteria of EORTC score for recurrence $<5$ may be considered for SIC (available at http://www.eortc.be/tools/bladdercalculator/) (LE 3; weak recommendation). The benefit of SIC for high-risk patients with multiple and large tumours who are planned for further BCG treatment is unclear, as patients with intermediate and high-risk disease in the study by Bosschieter et al were treated with adjuvant chemotherapy rather than BCG (LE 3) ${ }^{228}$.

Although heterogeneous, studies on SIC seem to agree that the timing of post-operative instillation is important. The majority suggests that the benefit is more obvious when SIC is done within the first 24 hours after the TURBT, with a trend for lower recurrence rates when instillation is delivered even earlier (LE 1; strong recommendation) ${ }^{233,234}$. Non-randomized comparisons taken from the same study by Sylvester et al in 2016 suggested that an instillation within 2 hours after TURBT was more effective than beyond this timepoint, supporting the rationale that the earlier the SIC is given post-TURBT, the more effective a SIC will be in reducing reimplantation and early recurrences ${ }^{227}$.

Recently, Onishi et al randomized 227 patients with low- and intermediate-risk NMIBC (mainly single LG Ta tumours $<3 \mathrm{~cm}$ ) to receive post-TURBT continuous instillation of normal saline $(2,000 \mathrm{~mL} / \mathrm{h}$ in the first hour and then $500 \mathrm{~mL} / \mathrm{h}$ for the following 15 hours $)$ versus a single instillation of MMC (30mg in $30 \mathrm{~mL}$ of saline for 1 hour $)^{235}$. With a median followup of 37 months, RFS at 3 years was comparable $(\mathrm{p}=0.53)$ and no difference in PFS was observed between groups ${ }^{235}$. This was further explored by 2 meta-analyses that showed no significant difference in RFS for immediate bladder irrigation with saline versus chemotherapy, although limited by a low number of studies ${ }^{236,237}$. Therefore, saline irrigation might be a consideration for patients with low- and intermediate-risk NMIBC post-TURBT when intravesical chemotherapy is contraindicated (e.g., extensive bladder resection) or unavailable (LE 2; weak recommendation).

\subsubsection{Adjuvant intravesical chemotherapy}

- Patients with intermediate-risk NMIBC should be considered for adjuvant induction intravesical chemotherapy ( $L E 1$; strong recommendation) with subsequent 
monthly maintenance for up to 1 year ( $L E 3$; weak recommendation), or induction BCG with maintenance therapy (refer to section 6.2).

- Substratification of intermediate-risk patients with recurrent LG Ta NMIBC can be used to guide adjuvant treatment decisions ( $L E 3$; weak recommendation). For this purpose, 4 factors should be considered: number of tumours, $\operatorname{size}(\geq 3 \mathrm{~cm})$, time to recurrence (< 1 year) and frequency of recurrence $(>1 /$ year).

○ Patients with low-intermediate-risk NMIBC ( 0 factors) may be treated similarly to low-risk patients, with SIC alone ( $L E$ 3; weak recommendation).

○ Patients with high-intermediate-risk NMIBC ( $\geq 3$ factors) may be treated as high-risk patients with induction and maintenance BCG (LE 3; weak recommendation).

- Patients who develop recurrence during intravesical chemotherapy may be offered induction followed by maintenance BCG ( $L E$ 3; weak recommendation).

No further treatment is needed other than SIC following TURBT for low-risk patients, while intermediate and high-risk patients should be considered for additional intravesical therapy. Several RCTs have shown lower rates of recurrence for patients undergoing induction intravesical chemotherapy with or without maintenance therapy, using either MMC, epirubicin, doxorubicin or pirarubicin, compared to TUR alone (LE 1) ${ }^{238-241}$.

Nevertheless, RCTs comparing induction only versus induction intravesical chemotherapy followed by maintenance have shown conflicting results ${ }^{221,240,242-245}$. A metaanalysis by Huncharek included 3,703 patients from 11 RCTs designed to study a benefit of adjuvant intravesical chemotherapy with followup duration of at least 1 year following TURBT $^{246}$. This study suggested a reduction in recurrence rates of $31 \%$ at 2 years and $73 \%$ at 3 years for patients undergoing maintenance for a duration of 1 and 2 years, respectively ${ }^{246}$. Conversely, 2 more recent meta-analyses revisited this topic and showed a more modest effect of maintenance intravesical chemotherapy in recurrence rates. The schedule and duration of intravesical chemotherapy was studied by Sylvester et $\mathrm{al}^{247}$. They highlighted significant heterogeneity among these studies regarding different regimens, schedules and duration of maintenance intravesical chemotherapy, concluding that no high-level recommendations were possible due to conflicting data ${ }^{247}$. The most recent meta-analysis sought to evaluate the added benefit of maintenance intravesical chemotherapy (most commonly for 7-12 months; range 3-36 months) versus induction intravesical chemotherapy alone for intermediate- and high-risk patients $^{248}$. This study showed that epirubicin, doxorubicin and MMC were the most commonly studied agents for maintenance intravesical chemotherapy but results among RCTs were inconsistent (out of 16 RCTs, only 3 showed significant benefit). The authors concluded that high heterogeneity between studies resulted in insufficient statistical power to show a significant 
benefit of maintenance intravesical chemotherapy compared to induction alone regarding recurrence, progression and survival rates ${ }^{248}$. Although further studies are needed to confirm the effect, ideal dose, schedule and recommended duration of maintenance intravesical chemotherapy, the approach of a single instillation monthly for up to 1 year might be considered for intermediate and high-risk patients in which an initial response to induction therapy was achieved after TURBT (LE 3; weak recommendation).

When adjuvant intravesical chemotherapy was compared to BCG, data from RCTs and meta-analyses suggested a reduced risk of recurrence (but not progression) with BCG for intermediate and high-risk patients (LE 1) at the cost of higher toxicity ${ }^{249-251}$. In an individual patient data meta-analysis of 2,820 patients with primarily papillary disease, where $74 \%$ were intermediate-risk, Malmstrom et al found a 32\% reduction in recurrence rates with BCG induction plus maintenance when compared to induction $\mathrm{MMC}$, while BCG without maintenance was inferior to induction MMC (LE 1) ${ }^{252}$. Moreover, progression and long-term survival rates were available for 1,880 patients but not statistically different across groups. A Cochrane review reached similar conclusions regarding likely reduced recurrence rates but greater serious adverse events with BCG versus MMC, with no significant difference in progression risk, although this meta-analysis highlighted that the certainty of the evidence was low ${ }^{253}$.

Intermediate-risk disease accounts for $35 \%$ of all cases of NMIBC and represents a heterogeneous subgroup of patients. In 2010, a substratification of intermediate-risk patients with recurrent LG Ta disease was proposed by Lamm et al and further adopted by the International Bladder Cancer Group (IBCG) ${ }^{254}$. Four factors were considered: number of tumours (multiplicity), tumour size $(>3 \mathrm{~cm})$, early recurrence $(<1$ year) and recurrence frequency ( $>1$ per year). Patients with none of the factors are classified as having "low-intermediate-risk" disease and can be managed similarly to low-risk patients with SIC. Those with 1-2 factors are considered true "intermediate-risk" patients and should be managed with adjuvant intravesical chemotherapy (induction followed by monthly maintenance for 1-year) or BCG (induction followed by that maintenance for 1 year with weekly instillations for 3 weeks at 3, 6 and 12 months). Finally, patients with $\geq 3$ factors are classified as "high-intermediate-risk" group, which are at higher risk for recurrence and progression and should be treated as high-risk patients, with full BCG schedule (induction followed by maintenance for 3 years with weeks instillations for 3 weeks at $3,6,12,18,24,30$, and 36 months $)^{255}$. Similarly, intermediate-risk patients with primary, small, and solitary HG Ta should be treated as high-risk patients with induction BCG followed by maintenance therapy.

In the previously cited meta-analysis by Malmstörm et al, BCG was effective even in patients receiving prior chemotherapy instillations ${ }^{252}$. Failure of adjuvant intravesical chemotherapy in intermediate-risk patients warrants consideration for BCG induction plus maintenance. 


\subsubsection{Device-assisted therapy \\ - Although intravesical chemotherapy through device-assisted therapy have shown promising results in small RCTs, further studies are needed to validate its routine clinical use.}

Electromotive drug administration (EMDA) is capable of increasing the uptake of drugs by cancer cells through electric current. It was tested with MMC, either in monotherapy or in association with BCG for high-risk NMIBC with promising results ${ }^{256-258}$. Di Stasi et al randomized 212 patients with BCG-naïve T1 NMIBC to undergo 6 weeks BCG induction versus 9 weeks of sequential weekly BCG (weeks 1, 2, 4, 5, 7, 8) and EMDA-MMC (weeks 3, 6 and 9)

257 . Monthly maintenance therapy was given in each arm to complete responders. With a median followup of 88 months, BCG + EMDA-MMC was associated with longer disease-free interval (69 versus 21 months, $\mathrm{p}=0.0012)$, lower rates of progression $(9.3 \%$ versus $21.9 \%, \mathrm{p}=0.005$ ) and lower overall mortality $(21.5 \%$ versus $32.4 \%, \mathrm{p}=0.045)$ compared to BCG alone. Side effects were acceptable in both treatment groups ${ }^{257}$. These results have not been validated in a second trial and, although approved by Health Canada, EMDA has not been widely adopted. Of note, EMDA catheters are no longer available in Canada.

Chemohyperthermia (CHT) with intravesical MMC has been studied for NMIBC. Radiofrequency elevates the temperature of the urothelium to $41-44^{\circ} \mathrm{C}$ while intravesical chemotherapy is delivered. Colombo et al enrolled 75 patients with primary or recurrent NMIBC and compared MMC alone to CHT-MMC, with both induction and maintenance schedules. Recurrence rates at 24-month followup were lower for the CHT-MMC group when compared to MMC alone $(17.1 \% \text { versus } 57.5 \% \text {, respectively; } \mathrm{p}=0.002)^{259}$. The authors also published a longterm update with median FU of 91 months and a sustained effect was seen in recurrence rates $(15 \% \text { vs } 53 \%, \mathrm{p}<0.001)^{260}$. A multicentre randomized trial by Arends et al with 190 intermediate and high-risk NMIBC patients compared 1-year CHT (induction + maintenance) vs 1-year BCG (induction + maintenance). In this study, intention-to-treat analysis was unable to prove any benefit of CHT-MMC over BCG on RFS at 24 months of followup (78.1\% vs $64.8 \%$, respectively $-\mathrm{p}=0.08$ ), highlighting the need for larger studies on device-assisted therapies in the first-line setting ${ }^{261}$. Although promising, especially in the context of chronic BCG shortage, CHT with MMC is not Health Canada approved and needs validation in larger studies before being integrated into routine clinical practice for high-risk NMIBC.

\subsection{BCG}

- In patients with high-risk NMIBC, BCG therapy with induction (weekly instillations for 6 weeks) followed by 3-year maintenance (weekly instillations for 3 weeks at 3, 6, 
$12,18,24,30$ and 36 months) is the standard of care for reducing disease recurrence and progression rates ( $L E 1$; strong recommendation).

- When BCG is administered for intermediate-risk NMIBC, induction (weekly instillations for 6 weeks) followed by 1-year maintenance (weekly instillations for 3 weeks at 3, 6 and 12 months) is recommended ( $L E$ 1; strong recommendation).

\subsubsection{Oncological outcomes and BCG}

When administered intravesically, BCG was proven to reduce recurrence rates of bladder cancer for the first time in 1976 by Morales et al ${ }^{262}$. During the mid 1980's and 1990's, 6 controlled trials confirmed a significant decrease in recurrence rates of up to $67 \%$ when tumour resection

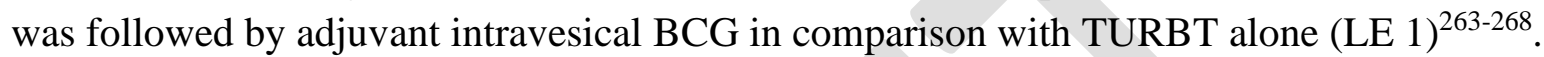
Meta-analyses including these studies confirmed a clear benefit of BCG in decreasing rates of recurrence compared to either TURBT alone or TURBT followed by different regimens of intravesical chemotherapy, with the greatest benefit reported among patients with high-risk NMIBC (LE 1; strong recommendation) $)^{249,251,269-272}$.

BCG has been the standard of care for decreasing not only recurrence, but also progression rates for high-risk disease. Initial studies by Herr and colleagues demonstrated a reduction in progression rates, from $35 \%$ to $28 \%$ in patients treated with TURBT alone versus TURBT plus BCG induction and also better OS outcomes (mortality rates of $32 \%$ versus $14 \%$ ) favouring $\mathrm{BCG}^{273}$. Later, Sylvester et al, in a meta-analysis including 4,863 patients from 24 RCTs, showed that $9.8 \%$ of patients progressed in the BCG group versus $13.8 \%$ in controls, reflecting a $27 \%$ relative reduction in the odds of progression (OR $0.73, p=0.001)$ in favour of $\mathrm{BCG}^{270}$. Notably, the benefit of BCG over other intravesical regimens was seen only in patients treated with BCG maintenance, rather than induction only (LE 1$)^{270}$.

For concurrent or primary CIS, BCG is also the standard of care, as it eradicates CIS and reduces risk of both recurrence and progression. Subgroup analysis of an early RCT in 1983 on patients with CIS demonstrated complete disease remission in $65 \%$ of patients undergoing TURBT plus BCG (median 18 months) versus $8 \%$ for TURBT alone (median 3 months) ${ }^{274}$. The meta-analysis by Sylvester et al in 2002 highlighted that the benefit of BCG in progression was seen for both papillary tumours and CIS ${ }^{270}$. In 2005, another meta-analysis by the same group compared patients with CIS treated with intravesical chemotherapy (MMC, epirubicin, doxorubicin or sequential MMC / doxorubicin) versus $\mathrm{BCG}^{272}$. They found that treatment failure and progression rates were higher with intravesical chemotherapy regimens compared with BCG $(\operatorname{LE~} 1)^{272}$. 


\subsubsection{BCG schedule, dose and strains}

Two-hour intravesical instillation of weekly induction therapy with BCG is usually administered over 6 weeks starting 2 to 4 weeks after TURBT to avoid systemic symptoms ${ }^{262}$. In the SWOG 8507 trial, 550 patients with recurrent NMIBC were randomized to BCG induction therapy with or without maintenance ${ }^{275}$. BCG maintenance was administered weekly over 3 weeks at 3, 6, 12, 18, 24, 30 and 36 months, counting from the beginning of induction therapy. Median RFS was 76.8 months for maintenance BCG versus 35.7 months for induction BCG only $(\mathrm{p}<0.0001)^{275}$. In this study, 278 patients had CIS. Although the study was not designed to assess BCG response for CIS patients, 6 month overall response rates were significantly improved for those treated with maintenance therapy when compared to induction only $(83.8 \%$ versus $68.1 \%$, respectively; $\mathrm{p}=0.004-\mathrm{LE} \mathrm{3})^{275}$. EORTC 30911 was a multicentre RCT with 957 intermediate and high-risk patients, (Ta/T1 only), designed to compare BCG versus epirubicin using induction and maintenance therapy in both arms. BCG was superior to epirubicin with respect to time to first recurrence $(\mathrm{p}<0.001)$, distant metastasis $(\mathrm{p}=0.046)$, OS $(\mathrm{p}=0.023)$, and DSS $(\mathrm{p}=0.026)$. Moreover, the relative benefit of BCG over epirubicin was at least as great in intermediate-risk as in high-risk patients ${ }^{250}$.

The EORTC 30962 was a non-inferiority trial that sought to evaluate if duration and dose of BCG maintenance could be reduced to 1 -year (versus 3 years) and $1 / 3^{\text {rd }}$ dose (versus full dose), respectively. The study found that $1 / 3^{\text {rd }}$ dose plus 1 -year maintenance was inferior to fulldose plus 3 years of maintenance BCG. However, there was no evidence that patients with intermediate-risk disease receiving full-dose derived any benefit with 3-years compared to 1-year of maintenance BCG. In contrast, for high-risk patients receiving full dose, 3-years of maintenance was superior to 1 year ${ }^{276}$. Meanwhile, an RCT by the CUETO group with 500 patients showed no difference in RFS, DFS and OS among patients treated with full dose BCG $\left(81 \mathrm{mg}\right.$ ) versus $1 / 3$ dose $(27 \mathrm{mg})^{277}$. However, for multifocal disease, the full dose was associated with lower recurrence and progression rates. Nevertheless, the results of the CUETO trial should be analyzed with caution as this study was considered statistically underpowered to prove equivalence or non-inferiority with clinically meaningful margins (LE 2) ${ }^{277}$.

The more recent NIMBUS trial was a phase III randomized study with $\mathrm{HG}$, recurrent or primary NMIBC in the BCG-naïve setting, including CIS patients ${ }^{278}$. The study compared standard induction plus maintenance (SWOG protocol) versus reduced frequency BCG therapy, in which induction was delivered with once-weekly BCG instillations at weeks 1, 2 and 6, while maintenance was delivered with single instillations at weeks 1 and 3 of months 3,6 and 12 . This study was halted early as a result of an inferior efficacy of the reduced schedule. Median followup was 12 months and a relative risk reduction for recurrence of $60 \%$ was reported for the standard versus reduced BCG schedule. Early separation of the Kaplan-Meier curves suggests that the reduced dose during induction therapy might have resulted in the significant impact on 
the efficacy of BCG for high-risk patients rather than duration of maintenance ${ }^{278}$. Taking into account all data, for intermediate-risk patients treated with BCG, the recommended schedule is induction followed by 1-year maintenance, while the 3-year schedule is recommended for highrisk patients (LE 1; strong recommendation).

Lastly, there are currently several strains of BCG available. Although differences in efficacy between strains have been proposed, a recent network meta-analysis was unable to confirm the association of different BCG strains with oncological outcomes ${ }^{279-284}$.

\subsubsection{BCG toxicity}

Although BCG is associated with more adverse effects than intravesical chemotherapy, serious toxicity occurs in only $5 \%$ of patients ${ }^{270,285}$. The most common local side effect of BCG instillation is the development of cystitis-like symptoms (urgency, dysuria and increased urinary frequency) that can be present in up to $71 \%$ of patients ${ }^{253}$. In such instances, a urine culture should be performed to rule out urinary tract infection ${ }^{286}$. Hematuria can occur and delay BCG instillations. If hematuria persists, cystoscopy to rule out early tumour recurrence or tumour persistence may be warranted. Epididymitis and prostatitis are less frequent effects, occurring in $10 \%$ and $3 \%$ of the cases, respectively ${ }^{287,288}$.

The most common systemic effect is fever, usually mild $\left(<38.5^{\circ} \mathrm{C}\right)$, lasting for less than 48 hours and accompanied by malaise and nausea. Persistent $(>48 \mathrm{~h})$ and high fever $\left(\geq 38.5^{\circ} \mathrm{C}\right)$ should prompt a complete workup for infection. Allergic reactions develop in less than $1 \%$ of the patients undergoing intravesical BCG and are treated with antituberculous drugs ${ }^{288}$. BCG sepsis requires hospitalization and active treatment (LE 3; strong recommendation) ${ }^{287}$. Adverse effects of BCG and their suggested management are described in Table 6.

\begin{tabular}{|l|l|}
\hline Table 6. BCG adverse events and recommendations \\
\hline Local side effects & Management \\
\hline LUTS / cystitis & $\begin{array}{l}\text { Mild and <48h: Symptomatic treatment with NSAIDs, phenazopyridine and } \\
\text { anticholinergics - resume treatment if symptoms improve. } \\
\text { Persistent: suspend BCG until symptoms improved or consider dose reduction; } \\
\text { laboratory workup (urine, blood, cultures); treat urinary tract infections with } \\
\text { culture-directed antibiotics; may consider short-term INZ 300mg/day } \pm \\
\text { corticosteroids. }\end{array}$ \\
\hline $\begin{array}{l}\text { Visible } \\
\text { Hematuria }\end{array}$ & $\begin{array}{l}\text { Suspend BCG until resolved; laboratory workup (urine, blood, cultures); culture- } \\
\text { directed antibiotics for urinary tract infection, if present; if hematuria is } \\
\text { persistent, then perform cystoscopy to rule out bladder cancer recurrence. }\end{array}$ \\
\hline $\begin{array}{l}\text { Epididymitis / } \\
\text { Prostatitis }\end{array}$ & $\begin{array}{l}\text { Suspend BCG; laboratory workup (urine, blood, cultures); add antibiotics (e.g., } \\
\text { quinolones); consider INZ 300mg/day or RFP 600mg/day; consider infectious } \\
\text { disease consultation; consider orchiectomy. }\end{array}$ \\
\hline & \\
\hline
\end{tabular}




\begin{tabular}{|l|l|}
\hline Systemic side effects & Management \\
\hline $\begin{array}{l}\text { Malaise / Nausea } \\
\text { usually < 48h) }\end{array}$ & Symptomatic treatment (e.g., antiemetics). \\
\hline Allergic reactions & $\begin{array}{l}\text { Mild and <48h: antihistamines; NSAID - delay BCG until resolved. } \\
\text { Persistent: Suspend BCG and consider discontinuing treatment; consider INZ } \\
\text { 300mg/day or RFP 600mg/day. }\end{array}$ \\
\hline Fever & $\begin{array}{l}\mathbf{< 3 8 . 5}^{\mathbf{O}} \mathbf{C} \text { and/or } \mathbf{4 8 h} \text { : symptomatic treatment (e.g., antipyretics). } \\
\mathbf{2} \mathbf{3 8 . 5}^{\mathbf{0}} \mathbf{C} \text { for } \mathbf{2 4 8 h} \text { : suspend BCG until resolved / consider dose reduction; } \\
\text { laboratory workup (urine, blood, cultures); start with at least } 2 \text { empiric } \\
\text { antimicrobials (e.g., quinolones, INZ 300mg/day, RFP 600mg/day); consider } \\
\text { infectious disease consultation. }\end{array}$ \\
\hline BCG sepsis & $\begin{array}{l}\text { Suspend BCG definitively; hospitalization; laboratory workup (urine, blood, } \\
\text { cultures); start empiric antibiotics (e.g., high-dose quinolones); initiate INZ } \\
\text { 300mg/day + RFP 600mg/day + ethambutol 1200mg/day, for 6 months); high- } \\
\text { dose corticosteroids if persistent (e.g., prednisolone 40mg/day). Infectious } \\
\text { disease consult. }\end{array}$ \\
\hline
\end{tabular}

*Modified from 287,291-294. BCG: bacillus Calmette-Guérin; INZ: isoniazide; LUTS: low urinary tract symptoms; NSAID: non-steroidal anti-inflammatory drug; RFP: rifampicin; UTI: urinary tract infection.

One study has evaluated whether toxicity is reduced if prophylactic antibiotics (ofloxacin) are administered following intravesical BCG therapy ${ }^{289}$. Although adverse local and systemic effects were reduced from $83.3 \%$ to $61.1 \%(\mathrm{p}=0.017)$ and from $75.9 \%$ to $54.4 \%$ $(\mathrm{p}=0.019)$, respectively, further studies are needed to validate the systematic use of antibiotics for this purpose ${ }^{289}$. Furthermore, a study comparing BCG versus epirubicin was unable to show a role of prophylactic isoniazid in reducing side effects during BCG schedule ${ }^{290}$. Other maneuvers that may be utilized in patients who poorly tolerate BCG include decreasing BCG dose and/or intravesical dwell time.

\subsubsection{BCG failure}

Despite adequate BCG administration, up to $40 \%$ of high-risk patients will experience long-term recurrence within 5 years ${ }^{295}$. Three different categories of BCG failure have been defined in the past:

1. BCG refractory includes any HG T1 after one induction course at 3 month followup, or any HG Ta or CIS after induction plus one round of maintenance or a second course of induction BCG at 6 month followup ${ }^{296}$. Patients with BCG refractory disease are at increased risk of progression and worse 5-year survival rates when compared to patients with a complete response to induction therapy (LE 3) ${ }^{297,298}$.

2. BCG relapsing disease is defined based on achieving a complete response to BCG treatment at 6 months but then experiencing any HG recurrence during followup 
thereafter. The prognosis of these patients improves with duration of the disease-free period after the last dose of $\mathrm{BCG}$, and their prognosis is better than patients with $\mathrm{BCG}$ refractory NMIBC. Therefore, patients with relapses before versus beyond 12 months of completing BCG are classified as early versus late relapse, respectively.

3. BCG-intolerant patients are those who experience recurrences after an inadequate course of BCG due to severe adverse effects. These patients have the best prognosis among BCG failure subcategories.

In order to standardize $\mathrm{BCG}$ failure and increase homogeneity among patients who are unlikely to respond to further intravesical BCG therapy, the term 'BCG-unresponsive' NMIBC was developed by a consensus panel representing experts from the American Urological Association and the US Food and Drug Administration (www.fda.gov/media/101468/download) (Table 7). Additionally, BCG-unresponsive disease implies patients being previously treated with adequate BCG schedule, defined as: at least 5-6 weekly instillations of an induction course followed by at least one maintenance cycle (consisting of at least 2 out of 3 weekly BCG instillations) or a second induction cycle (whereby at least 2 of 6 weekly instillations were received).

BCG-unresponsive bladder cancer includes patients meeting any one of the following criteria:

- HG T1 at the first evaluation following induction BCG (at 3 months).

- Recurrent HG Ta/T1 within 6 months of completion of adequate BCG treatment.

- Recurrent CIS (+/- Ta/T1) within 12 months of completing adequate BCG treatment.

\begin{tabular}{|l|l|}
\hline Table 7. BCG failure classification \\
\hline $\begin{array}{l}\text { BCG failure } \\
\text { stratification }\end{array}$ & Definition $^{\dagger}$ \\
\hline BCG-unresponsive & $\begin{array}{l}\text { HG T1 at the first evaluation following induction BCG (3 months); } \\
\text { Recurrent HG Ta/T1 within 6 months of adequate BCG treatment; } \\
\text { Recurrent CIS within 12 months of adequate BCG treatment* }\end{array}$ \\
\hline BCG refractory & $\begin{array}{l}\text { HG T1 at the first evaluation following induction BCG (3 months); } \\
\text { Persistent/recurrent HG Ta/CIS following adequate BCG (6 months) }\end{array}$ \\
\hline BCG relapsing & $\begin{array}{l}\text { HG recurrence after reaching a disease-free state within 6 months of } \\
\text { receiving adequate BCG }\end{array}$ \\
\hline BCG intolerant & $\begin{array}{l}\text { Disease recurrence/persistence after failure to receive adequate BCG } \\
\text { therapy due to severe adverse effects. }\end{array}$ \\
\hline
\end{tabular}

${ }^{\dagger} \mathrm{By}$ definition, low-grade recurrences during or after BCG are not considered BCG failure. *Adequate BCG - at least 5-6 weekly induction courses followed by at least one maintenance cycle (consisting of at least 2 out of 3 weekly BCG treatments) or a second induction cycle (whereby at least 2 of 6 weekly instillations were received). BCG: bacillus Calmete-Guérin; CIS - carcinoma in situ; HG: high-grade. 


\subsubsection{Management of BCG-unresponsive NMIBC}

$\mathrm{RC}$ with pelvic lymph node dissection is the standard of care for BCG-unresponsive bladder cancer in surgically fit patients ( $L E 3$; strong recommendation). For patients with BCG-unresponsive CIS or HG Ta, a second-line intravesical therapy might be considered before $\mathbf{R C}$ ( $L E$ 3; weak recommendation).

- Promising efficacy has been reported with intravenous pembrolizumab, intravesical oportuzumab monatox, nadofaragene firadenovec, and BCG plus N-803. These should be considered as potential options in patients with BCG-unresponsive CIS who are unfit for or refuse to undergo $\mathbf{R C}$ ( $L E 2$; weak recommendation).

- Alternative options such as sequential intravesical gemcitabine/docetaxel (induction plus maintenance) may be considered for patients with BCG-unresponsive disease who are unfit for or refuse to undergo $\mathbf{R C}$ ( $L E$ 3; weak recommendation). Additional alternatives may also include other combination intravesical therapy (e.g., sequential gemcitabine/MMC, BCG + interferon if available) or single-agent intravesical therapy (MMC, epirubicin, docetaxel, gemcitabine) ( $L E$ 3; weak recommendation).

- Clinical trials may be considered for BCG-unresponsive patients who are unfit for or refuse to undergo $\mathrm{RC}$.

a) Radical cystectomy with pelvic lymphadenectomy

$\mathrm{RC}$ is the standard for patients with BCG-unresponsive NMIBC, and studies have shown that patients with recurrent disease benefit from early radical surgery (LE 3; strong recommendation). A study by Herr et al evaluated a subset of 90 patients that underwent cystectomy after failing BCG therapy ${ }^{299}$. With a followup of 96 months, survival rates among patients undergoing RC within 2 years of the initial BCG therapy was of $92 \%$ versus $56 \%$ for patients having surgery after 2 years of the initial BCG therapy. Early RC was also confirmed as an independent predictor of survival in multivariable analysis (LE 3) ${ }^{299}$. Patients considered surgically unfit and those unwilling to undergo RC should be counselled regarding higher risks of recurrence and progression associated with bladder-preserving alternatives. Whenever available, enrolment in a clinical trial should also be considered (LE 3; weak recommendation).

Although patients with BCG-unresponsive HG T1 are recommended for RC (LE 3; strong recommendation), those with BCG-unresponsive CIS or HG Ta may be offered an attempt for second-line bladder-sparing therapy prior to $\mathrm{RC}$, as retrospective studies and recent singlearm trials suggest that such patients can be managed conservatively for up to one year after initial TURBT without an impact on cancer-specific mortality (LE 3; weak recommendation) ${ }^{300-}$ 302 . 


\section{b) Single-agent adjuvant therapies - chemotherapy}

\section{Valrubicin}

Steinberg et al enrolled 90 patients with recurrent CIS after multiple courses of intravesical therapy (including at least one BCG course), to receive weekly $800 \mathrm{mg}$ of intravesical valrubicin for 6 weeks ${ }^{303}$. Median followup was 30 months and complete response at 3 and 6 months was seen in 19 (21\%). Complete responders had a median time to recurrence of 18 months and recurrences were registered in $88 \%$ of patients at the end of this study's followup ${ }^{303}$. Similar initial responses for valrubicin were found in another study with CIS patients failing BCG, but the long-term disease-free rate was $10 \%$ and $4 \%$ at 12 and 24 months, respectively ${ }^{304}$. Although valrubicin was the only approved agent in the US (until recently) for patients with BCG refractory CIS, it has rarely been used in clinical practice.

\section{Other}

Laudano et al published on long-term outcomes of a phase I trial with induction docetaxel in 18 patients with recurrent NMIBC with no toxicities reported ${ }^{305}$. More recently, a study by Barlow et al included 54 patients with BCG refractory NMIBC to receive induction intravesical docetaxel (weekly dose for 6 weeks) followed by maintenance (monthly dose for up to 9 months) if an initial complete response was achieved. With a median followup of 39 months, complete response was reported in 59\% of patients. RFS rates at 1 and 3 years were $40 \%$ and $25 \%$, respectively ${ }^{306}$. Similarly, intravesical gemcitabine was studied in a phase I trial with 18 patients with BCG-refractory disease also showing a favourable toxicity profile ${ }^{307}$. SWOG S0353 was a multicentric phase II trial assessing intravesical gemcitabine in recurrent NMIBC (where 89\% were high-risk) after at least 2 courses of BCG. Fifty-eight patients were enrolled and treated with induction (2000mg of gemcitabine in 6-weekly instillations) followed by monthly dose maintenance for up to 12 months. Complete response was seen in $47 \%$ of patients at the first 3month assessment. RFS at 1 and 2 years were of $28 \%$ and $21 \%$, respectively (LE 2) ${ }^{308}$.

As other single-agent therapies also demonstrated only modest efficacy for BCGunresponsive disease ${ }^{309,310}$, combinations of drugs have been evaluated to increase response rates (see below).

\section{c) Device-assisted therapy}

Device-assisted therapy was also tested in the setting of BCG-unresponsive disease. Racioppi et al recently published the results of a phase II single-arm study on 26 patients using EMDAMMC (induction and maintenance) for high-risk NMIBC who failed BCG therapy ${ }^{311}$. Median followup was 36 months and $\mathrm{HG}$ disease-free rate was $61.5 \%{ }^{311}$. In this study, a total of 10 patients underwent RC, of those 6 (23.1\%) had recurrent HG NMIBC and 4 (15.4\%) had disease progression ( 3 patients with pT2 and 1 with pT4a) ${ }^{311}$. Moreover, a retrospective study by Juvet et al evaluated 26 patients who failed BCG (all, except four were classified as BCG-unresponsive) 
and were treated with sequential BCG and EMDA-MMC ${ }^{312}$. Complete response rates at 6, 12 and 18 months were $62 \%, 44 \%$ and $30 \%$, while PFS at 2 years was of $48 \%$. Importantly, $15 \%$ of patients died of bladder cancer after 2 years of followup, highlighting the potential risks of conservative management in this patient population ${ }^{312}$. Of note, EMDA catheters are no longer available in Canada.

A phase III RCT by Tan et al (HYMN) evaluated radiofrequency-induced CHT in patients with intermediate- or high-risk NMIBC recurrences who failed initial BCG therapy ${ }^{313}$. This trial was closed prematurely due to higher than expected CIS recurrence rates in patients treated with CHT and, overall, there was no benefit in 24-month DFS rates among the two groups $-35 \%$ versus $41 \%$ for CHT versus control $-(\text { HR } 1.33,95 \% \text { CI } 0.84-2.10, p=0.23)^{313}$. Therefore, until further studies evaluate the role of CHT in BCG-unresponsive disease, this option should only be offered to patients in the setting of a clinical trial.

\section{d) Combination intravesical therapies}

For BCG-unresponsive patients undergoing intravesical chemotherapy, sequential combination of drugs is favoured instead of single-agent regimens (LE 3; weak recommendation) ${ }^{314,315}$.

\section{Sequential gemcitabine + docetaxel}

Due to worldwide shortages of MMC during the late 1990's, sequential instillation of gemcitabine followed by docetaxel after TURBT has also been studied. Steinberg et al reported the first experience of this combination in 45 patients mainly with BCG refractory and relapsing disease $^{315}$. Treatment was generally well tolerated, and side effects were mild, although 5 patients were unable to undergo the full induction course with 6 weekly instillations. Response was achieved in $66 \%$ of patients at first assessment, while 1-year and 2-year RFS rates were 54\% and $34 \%$, respectively (LE 3$)^{315}$. Another study with 33 patients confirmed similar results with sequential gemcitabine-docetaxel-RFS at 1 and 2 years was of $56 \%$ and $42 \%$, respectively ${ }^{316}$.

More recently, a multicentric retrospective study reported on 275 patients, of whom $38 \%$ had BCG-unresponsive disease ${ }^{317}$. Induction consisted of weekly instillation of gemcitabine $(1 \mathrm{~g}$ in $50 \mathrm{~mL}$ of normal saline, instilled for $60-90 \mathrm{~min}$ ) followed by docetaxel $(37.5 \mathrm{mg}$ in $50 \mathrm{~mL}$ of normal saline, for 60-120 minutes) for 6 weeks. The majority (78\%) of patients with a complete response at 3 months were treated with monthly maintenance for 12-24 months. With a median followup of 22.9 months, RFS was $77 \%, 60 \%$ and $46 \%$ at 6,12 and 24 months, respectively. HG-RFS for BCG-unresponsive patients was 50\% at 24 months. In multivariable analysis, the only variable associated with increased RFS was the use of gemcitabine-docetaxel maintenance schedule. Overall, gemcitabine-docetaxel was well tolerated and considered effective by the authors as a rescue therapy in patients previously treated with BCG for NMIBC (LE 3; weak recommendation $)^{317}$. 


\section{Sequential gemcitabine $+M M C$}

The sequential combination of intravesical gemcitabine and MMC was prospectively investigated but studies were limited by a low number of patients and single-arm design. In a phase I trial including 10 BCG-refractory/intolerant patients, toxicity was acceptable and 6 were recurrence-free after median followup of 14 months $^{318}$. Later, Lightfoot et al reviewed 45 patients treated with induction of 6-weekly gemcitabine $(1000 \mathrm{mg}$ in $50 \mathrm{~mL}$ of sterile water, instilled for $90 \mathrm{~min}$ ) followed by MMC (40mg in $20 \mathrm{~mL}$ of sterile water, $90 \mathrm{~min}$ ) for 6 weeks, followed by monthly maintenance for up to 12 months. Treatment was overall well tolerated, a complete response was achieved in $68 \%$ of patients and RFS at 1 and 2 years was $48 \%$ and $38 \%$, respectively ${ }^{314}$. A recent series on 27 patients have shown similar results, with $37 \%$ (10) of patients with no evidence of disease at a median FU of 22 months ${ }^{319}$.

\section{$B C G+$ interferon-alpha}

Joudi et al studied the combination of BCG and interferon-alpha in a phase II trial. A total of 536 patients were BCG-naïve, while 467 were in the BCG-failure group (39\% previously treated with 2 or more BCG courses). Recurrence-free rates at median followup of 24 months was $45 \%$ and $59 \%$ for the BCG-failure and BCG-naïve subgroups, respectively ${ }^{320}$. Further analysis of this study suggested the combination of BCG + interferon-alpha might be a reasonable choice especially for patients relapsing more than 1 year after initial BCG treatment, but seems to be not effective in patients with BCG-refractory disease ${ }^{321}$. Therefore, BCG + interferon-alpha is not recommended for BCG-refractory patients who are at higher risk of recurrence, while it might be considered in patients with BCG relapse if available (LE 3; weak recommendation). Of note, interferon-alpha will no longer be available in 2021 as Merck has announced that it will soon discontinue its production.

\section{e) Novel agents}

\section{Pembrolizumab}

Pembrolizumab (Keytruda ${ }^{\circledR}$ ) is a PD-1 checkpoint inhibitor approved for metastatic bladder cancer patients in the $2^{\text {nd }}-$ line or first-line when cisplatin-ineligible ${ }^{322}$. It was also recently approved for BCG-unresponsive CIS in 2020 in the US (by the FDA) and in Canada by Health Canada, based on results from the Keynote-057 study (abstract) ${ }^{301}$. In this single-arm study, $200 \mathrm{mg}$ of pembrolizumab was administered intravenously every 3 weeks for 24 months or until recurrence, progression or limiting toxicity. Preliminary data on 96 patients with BCGunresponsive CIS was presented. Complete response was achieved in 40 patients $(40.6 \%, 95 \%$ CI $30.7-51.1 \%$ ) at 3 months and median duration of response was 16.2 months. Out of 39 responders, 18 (46.2\%) were recurrence-free at 12 months with no progression events; this translates to an overall 12-month complete response rate of $19 \%$ in the CIS cohort (LE 2) ${ }^{301}$. 
Treatment was well tolerated, and adverse effects were reported in 67 patients (65.7\%), out of which $13(12.7 \%)$ were grade 3 or $4^{301}$.

\section{Nadofaragene firadenovec}

rAd-INFa/Syn3 is a non-replicating adenovirus vector together with recombinant IFN-alpha2b, also known as nadofaragene firadenovec (Adstiladrin ${ }^{\circledR}$ ). When given intravesically, the virus is transduced into bladder cells and the IFN-alpha2b gene is incorporated by the DNA. IFNalpha $2 \mathrm{~b}$ protein, which has antitumour activity, is then produced. The first phase II, multicentre and open-label study including 43 patients with HG BCG-refractory or relapsed disease showed a RFS rate of $35 \%$ at 12 months ${ }^{323}$. Recently, the phase III study was published by Boorjian et al and reported on 151 patients with BCG-unresponsive disease ${ }^{302}$. After one initial instillation of nadofaragene firadenovec, patients with no high-grade recurrence received additional doses every 3 months up to a maximum of 4 years. Out of 103 patients with CIS (with or without HG $\mathrm{Ta} / \mathrm{T} 1$ ), complete response at 3 and 12 months was reported in $53.4 \%$ and $24.3 \%$, respectively. A durable response within responders was seen in $45.5 \%$ at 12 months. For papillary HG Ta/T1 disease with no CIS, 12-month complete response rate was $43.8 \%$. Nadofaragene firadenovec was well tolerated, with only $4 \%$ of patients experiencing drug-related grade 3 or 4 adverse effects $^{302}$.

\section{Oportuzumab monatox}

Oportuzumab monatox (Vicineum ${ }^{\circledR}$ ) is a specific antibody to Epithelial Cell Adhesion Molecule (EpCAM) fused to a Pseudomonas toxin that binds specifically to bladder cancer cells. A singlearm phase III trial (VISTA) was conducted in patients with BCG-unresponsive NMIBC ${ }^{324}$. Intravesical treatment was given with an induction course ( $2 \mathrm{x} /$ week for 6 weeks then weekly for 6 weeks) followed by a maintenance regimen (once every 2 weeks up to 2 years). Complete response rates for patients with CIS were $40 \%$ and $17 \%$, at 3 and 12 months, respectively. For papillary HG Ta/T1 disease with no CIS, 12-month disease-free rate was 50\%. Severe adverse effects were seen in 3 patients, but overall, the drug was well tolerated ${ }^{324}$.

BCG plus $N-803$

$\mathrm{N}-803$ is an IL-15 superagonist antibody cytokine fusion protein that can be co-administered intravesically with BCG to induce activation and proliferation of endogenous natural killer (NK) cells and CD8+ T-cells without inducing a T-reg response ${ }^{325}$. Interim analysis of the pivotal Phase II/III QUILT 3.032 single-arm trial was recently presented in the 2021 ASCO Genitourinary Cancers Symposium. In that trial, 80 patients with BCG-unresponsive CIS +/-

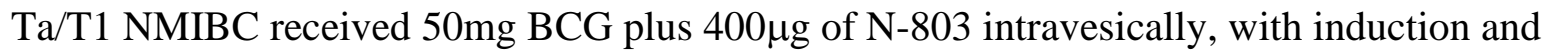
maintenance courses. Re-induction was allowed for persistent disease. At a median followup of 10.7 months, $71 \%$ of patients achieved a complete response, with a $56 \%$ probability of duration 
of complete response $>12$ months, and an $87.5 \%$ probability of not undergoing RC. The most common treatment-related adverse events were dysuria, hematuria, and pollakiuria (mostly grade 1-2). Final peer-review publication and regulatory review were still pending at the time of writing of this guideline.

Future perspectives for BCG-unresponsive disease

Other novel agents are being developed and tested in the BCG-unresponsive setting. Ongoing trials include FGFR 3 inhibitors, gene therapy, viral gene therapy, IDO1 inhibitors, interleukin antagonists, and vaccines ${ }^{326}$. A summary of the management of NMIBC is presented (Figure 1). Patients with HG NMIBC that recurs after some BCG therapy, but does not fulfill the FDA-AUA criteria of BCG-unresponsive disease were recently termed as 'BCG experienced' or 'BCG exposed'. BCG in combination with immunotherapy is being evaluated in such patients with persistent or recurrent HG disease or CIS after one single BCG induction course without maintenance ${ }^{327}$.

\section{f) Radiation therapy}

In the NMIBC setting, evidence for radiation-based therapy is scarce. Weiss et al published their retrospective series on 141 patients with primary or recurrent high-risk NMIBC who underwent radiotherapy (median dose 55.8Gy) and concurrent platinum-based chemotherapy (either cisplatin or carboplatin) $)^{328}$. Complete response was achieved in $88 \%$ of patients. Median followup was 62 months and failure rates, defined as any recurrence or progression, at 5 and 10 years were $49 \%$ and $64 \%$, respectively (LE 3). Among survivors, $80 \%$ were able to preserve their bladders, with acceptable toxicity and no treatment-related deaths ${ }^{328}$. Currently, RTOG0926 study (NCT00981656) has completed accrual and awaits readout on the role of trimodal therapy for patients with recurrent papillary high-grade Ta/T1 who fail BCG. Notably, data extrapolated from studies with chemoradiation for MIBC suggest that patients with concomitant CIS experience worse outcomes ${ }^{329}$. As a result, chemoradiation for NMIBC should not be recommended for patients with BCG-unresponsive CIS. 
Fig. 1. Flow chart for the management of patients with non-muscle invasive bladder cancer.

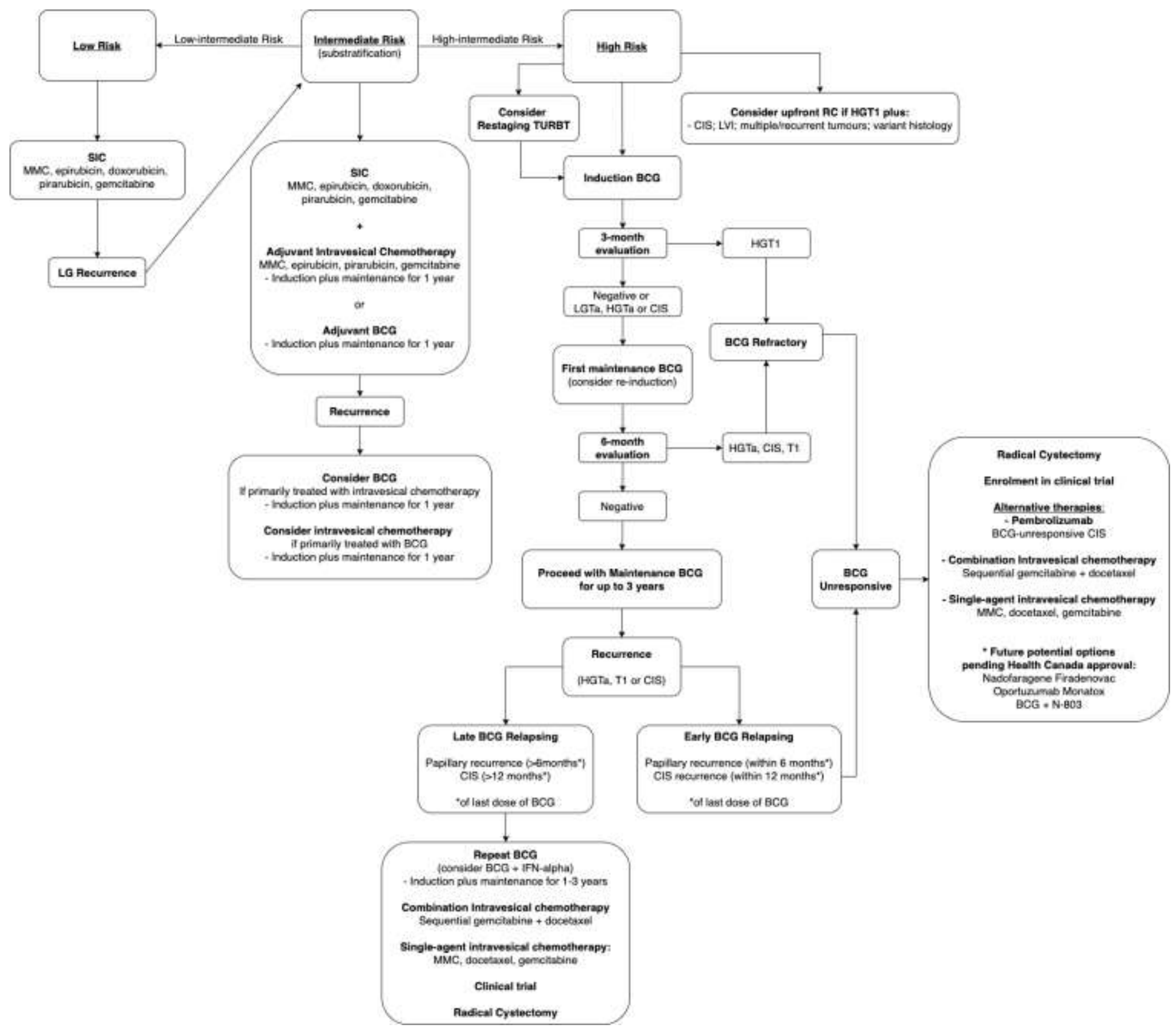

*Modified from Chehroudi et al ${ }^{330}$. BCG: Bacillus Calmette-Guérin; CIS: carcinoma in situ; GEM: gemcitabine; HG: high-grade; Ind: induction; LG: low-grade; LVI: lymphovascular invasion; MMC: mitomycin-C; RC: radical cystectomy; SIC: single instillation of chemotherapy; TURBT: transurethral resection of bladder tumour. 


\subsubsection{Treatment adjustments only if $B C G$ shortage}

- For patients with intermediate-risk NMIBC during BCG shortage, intravesical chemotherapy is recommended as the first-line option. If BCG is planned as a second-line therapy for this population, induction might be administered with reduced dose (1/2 or 1/3 dose) and maintenance can be omitted ( $L E 3$; weak recommendation).

- For patients with high-risk NMIBC, full BCG schedule (induction followed by 3year maintenance) is recommended ( $L E 1$; strong recommendation). Only during BCG shortage, when full dose is not possible due to limited supply, dose reduction to $1 / 2$ or $1 / 3$ might be considered, while maintenance can be reduced to one year $(L E 3$; weak recommendation).

- When BCG is unavailable, single agent chemotherapy (e.g., MMC, gemcitabine) or sequential combination of intravesical chemotherapy (e.g., gemcitabine/docetaxel) is recommended with induction followed by monthly maintenance for up to one year (LE 3; weak recommendation).

Between 2013 and 2016, global production of BCG decreased dramatically. A study conducted in France suggested that recurrence rates increased during that period when compared to previous years $(46.9 \%$ versus $16.2 \%$, respectively $-\mathrm{p}<0.001)(\mathrm{LE} 3)^{331}$. Costs also increase during a BCG shortage because alternative treatments are currently more expensive than $\mathrm{BCG}^{331,332}$. Therefore, this worldwide shortage remains a challenge. The shortage applies to different BCG strains and is mainly caused by slow production process, manufacturing issues and limited supplies, negatively affecting the ideal treatment of NMIBC. Urologists should be aware that alternatives in this scenario are continuously being studied and discussed by the scientific community.

In June 2019, the medical advisory board of Bladder Cancer Canada, together with the CUA Guidelines Committee released a document in response to continuous limited supply of the Tice strain of BCG in Canada (https://bladdercancercanada.org/wpcontent/uploads/2019/06/Approach-to-Address-BCG-Shortage-updated-June-2019.pdf).

Strategies to overcome a BCG shortage are here presented and stratified in two scenarios: BCG-restricted and BCG-unavailable.

BCG-restricted supply

Although optimized BCG schedules were studied with the purpose of keeping production sustainable and available for a higher number of patients, in the recently published NIMBUS trial, a reduced schedule of BCG was inferior to the standard schedule in patients with high-risk NMIBC $^{278}$. Moreover, the EORTC study by Oddens et al have shown that, although for intermediate-risk the one-year BCG maintenance schedule was not inferior to a 3-year schedule, 
high-risk patients benefited from the 3-year full schedule ${ }^{250}$. However, no difference in progression rates were noted across the treatment arms. Hence, during a shortage period with reduced BCG supplies, high-risk patients may receive a reduced induction BCG dose of $1 / 2$ or $1 / 3$ while maintenance therapy can also be reduced to 1 year instead of 3 years (LE 3; weak recommendation).

For intermediate-risk patients, intravesical chemotherapy in the first-line setting would be the preferred choice. Moreover, if BCG is used as second-line for intermediate-risk patients, reduced dose can be administered during BCG shortage, and maintenance therapy omitted (LE 3; weak recommendation).

\section{BCG-unavailable}

For high-risk patients, when BCG is not available, intravesical chemotherapy with MMC is an alternative, as its efficacy in patients with papillary disease may be similar to BCG with respect to progression and DSS according to some studies ${ }^{252,333}$. Other alternatives presented in this manuscript should also be considered, namely, hyperthermic or electromotive MMC, or single

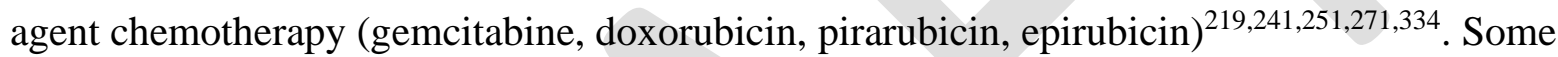
alternatives tested in the BCG-failure setting, such as sequential gemcitabine-docetaxel or gemcitabine-MMC, might also be explored during a BCG shortage in high-risk NMIBC, although further studies are needed to confirm their efficacy in the first-line BCG-naive setting (LE 3; weak recommendation) ${ }^{315,316,319}$. Importantly, patients with high-risk disease and additional higher risk features such as CIS, LVI, PUI or variant histology (namely micropapillary, plasmacytoid and sarcomatoid) should be counselled for upfront RC (LE 3; strong recommendation) or enrollment in clinical trials (weak recommendation).

\section{Timely cystectomy}

- Upfront RC should be considered for patients with large volume, diffuse, endoscopically unresectable NMIBC ( $L E$ 3; strong recommendation)

- Upfront RC should be offered to patients with HG T1 disease with additional adverse tumour pathological features including: variant histology (e.g., micropapillary, plasmacytoid, sarcomatoid), extensive invasion of the lamina propria or invasion into or beyond the muscularis mucosa $(\mathrm{T} 1 \mathrm{~b} / \mathrm{c})$, presence of $\mathrm{LVI}$, concomitant CIS in the bladder or prostatic urethra, multiple or large $(\geq 3 \mathrm{~cm})$ tumours and persistent HG T1 upon restaging TURBT ( LE 3; strong recommendation).

Timely RC is an important consideration for patients with NMIBC considered at higherrisk of progression ${ }^{299,300}$. Although the terms "upfront" and "early" RC have been used indistinctively in the literature, upfront refers to surgery offered at the time of NMIBC diagnosis, 
while early RC refers to patients with persistent or recurrent disease after initial intravesical treatment, but who were not yet diagnosed with $\mathrm{MIBC}^{335}$. Upfront $\mathrm{RC}$ is associated with higher cancer-specific survival at 10 years when compared to early RC (79\% versus $65 \%$, respectively) according to a retrospective study by Hautmann et al (LE 3$)^{336}$. The term "timely cystectomy" includes both scenarios when the attempt at bladder-sparing therapies is unsuccessful or inappropriate in high-risk patients ${ }^{335}$. Therefore, defining patients at the higher risk of progression is key not only to refine the indication for timely $\mathrm{RC}$, but also to avoid overtreatment in this population, since perioperative complications, morbidity, mortality and long-term quality of life are major concerns with $\mathrm{RC}^{337,338}$.

Several risk factors discussed along this guideline are known to be associated with higher rates of recurrence and, more importantly, progression (refer to section 3). For HG recurrent NMIBC despite adequate BCG therapy, any further attempt at bladder preservation is not ideal and early RC should be offered whenever feasible (strong recommendation). Furthermore, patients with de novo high-risk disease and additional high-risk features discussed in this guideline should also be offered timely RC (LE 3; strong recommendation), particularly those with variant histology (micropapillary, plasmacytoid and sarcomatoid) or the following risk factors: extensive or deep T1 invasion, multiple / large tumours, CIS (bladder and/or prostate), LVI, persistent T1 disease at restaging TURBT and pT1 recurrence (LE 4) ${ }^{339}$. Shared decisionmaking should take place with the patient, weighing the risk of cancer progression if treated without RC against the risk of over-treatment with RC.

A recent multicentre pilot feasibility study by Catto et al (BRAVO) evaluated the possibility of recruiting patients with high-risk NMIBC to undergo either upfront RC or BCG induction + maintenance ${ }^{340}$. Out of 407 screened patients, 215 were eligible and 50 were ultimately randomized during a pre-set period of 18 months. For this reason, recruitment was stopped due to failure to accrue a minimum 60 patients in 18 months as initially planned. Despite advanced age (> 70 years in $66 \%$ of patients) and smoking history (in $75 \%$ of patients), $80 \%$ of screened patients were deemed fit for RC and considered eligible. Quality of life was comparable among groups. Finally, this study showed that $25 \%$ of patients in the cystectomy arm were pT0 after final specimen pathology evaluation, possibly suggesting overtreatment. On the other hand, although NMIBC is considered a non-lethal disease by many urologists, up to $10 \%$ of patients in the RC group had MIBC in the final specimen of that study, highlighting the need for radical surgery in a minor subset of high-risk patients ${ }^{340}$.

\section{Followup}

- The first surveillance cystoscopy is recommended for all patients at 3 months after TURBT (LE 2; strong recommendation). 
- After the 3-month cystoscopy, a risk-based surveillance strategy should be used in patients with no evidence of recurrence:

○ Low-risk patients might be followed with cystoscopy at 1 year and then yearly for 5 years ( $L E 3$; weak recommendation). Urinary cytology is not necessary in the followup of low-risk patients ( $L E 4$; weak recommendation).

$\circ$ Intermediate-risk patients should be followed with cystoscopies and urine cytology every 3-6 months in the first 2 years, every 6-12 months in the $3^{\text {rd }}$ year, and annually thereafter ( $L E 3$; weak recommendation).

○ High-risk patients should be followed with cystoscopies and urine cytology every 3-4 months during the first 2 years, every 6 months during years 3 and 4 , and annually thereafter ( $L E 3$; weak recommendation).

- Upper tract imaging is recommended with random bladder / prostatic urethral biopsies (or use of BLC with directed biopsies) if positive urine cytology with normal cystoscopy is found during surveillance ( $L E 3$; weak recommendation).

- Upper tract imaging surveillance is recommended in the first year and every 2 years thereafter for high-risk patients ( $L E 3$; weak recommendation).

- Fulguration under local anesthesia might be considered for small $(<5 \mathrm{~mm})$ papillary tumours and negative cytology in patients with a prior history of PUNLMP or LG Ta tumours (LE 3; weak recommendation).

There is currently no high-level evidence on specific surveillance protocols for the different stages of NMIBC. Due to high rates of recurrences for low-risk and progression for high-risk patients, surveillance for NMIBC should be diligent and ideal followup consists of adapting strategies based on individual risk (LE 3) ${ }^{83,122}$. General recommendations are that all newly diagnosed patients treated with initial TURBT should undergo a first assessment at 3 months with voided urine cytology (cytology not required for patients with low-risk NMIBC) and cystoscopy. Cystoscopic and pathological findings at first 3-month assessment post-TURBT are associated with oncological outcomes (recurrence and progression), particularly for high-risk patients ${ }^{83,84,298}$. Additionally, urine cytology is a useful tool during surveillance for high-risk patients with sensitivity as high as $70-90 \%{ }^{77-80}$. For pTa disease, urine cytology yields sensitivity of $47 \%$, while for grade 1 and 2, rates of $27 \%$ and $54 \%$ were reported, respectively $(\operatorname{LE~} 3)^{341}$.

Importantly, followup schedule should restart if recurrent disease is identified during surveillance. Studies with long-term followup have shown that there is a significant decrease in recurrence rates after 5 years, particularly for LG Ta tumours ${ }^{342}$. Mariappan et al reported significantly lower recurrence rates after 5 years $(14.1 \%)$ compared to the first 5 years of surveillance $(29.1 \%, \mathrm{p}=0.009)$ in 115 patients with LG Ta tumours followed for a mean time 
period 19.4 years $^{343}$. Therefore, surveillance can be stopped after 5 years for patients with lowrisk NMIBC, if no evidence of recurrence, while long-term surveillance is recommended for patients with intermediate and high-risk disease (LE 3; weak recommendation). PUNLMP is associated with recurrence rates comparable to LG Ta tumours and progression is rare. Therefore, these patients should be followup similarly to LG tumours (LE 3; weak recommendation $)^{86}$.

A study by Millan-Rodriguez et al evaluated the incidence of UTUC after bladder cancer diagnosis in 1,529 patients. Overall, incidence was $2.6 \%$, but varied according to risk stratification from $1.6 \%$ (low-risk) to $4.1 \%$ (high-risk) ${ }^{344}$. Upper tract imaging is recommended in cases of gross hematuria or positive urine cytology with a normal appearing bladder at cystoscopy (LE 3; weak recommendation). Furthermore, population-based studies have suggested that patients with $\mathrm{T} 1$, multifocal and/or HG tumours are at 2.5-fold higher risk of recurrence in the upper tract (LE 3) ${ }^{345}$. Additionally, the presence of CIS / T1 tumours invading the intramural portion of the ureteral orifices confers higher risk of UTUC (HR 6.85, p $=0.005$ and HR 7.25, $\mathrm{p}=0.001$, respectively $)^{346}$. Therefore, high-risk NMIBC patients should undergo CT and intravenous urography every 1-2 years (LE 3; weak recommendation), while high-level evidence is lacking regarding on how long upper tract surveillance should be continued.

\section{Fulguration under local anesthesia}

Fulguration under local anesthesia is a suitable alternative to TURBT for patients with prior LG Ta tumours who have a small $(<5 \mathrm{~mm})$ recurrent papillary tumour found on surveillance cystoscopy. It is associated with similar recurrence rates when compared to TURBT, but with lower rates of complications and at a lower $\cos ^{347-349}$. This procedure might be considered in the surveillance of previously low-risk and select intermediate-risk patients (LE 3; weak recommendation) ${ }^{350,351}$.

More recently, active surveillance has been proposed for asymptomatic, few $(\leq 3)$ and small $(\leq 10 \mathrm{~mm})$ papillary tumours with negative urine cytology and a known history of LG Ta tumours. Although considered potentially safe in initial studies, additional prospective studies are needed to identify ideal candidates and define surveillance protocols for this approach ${ }^{352}$. Based on the current literature, a risk-stratified surveillance schedule is proposed (Table 8). 


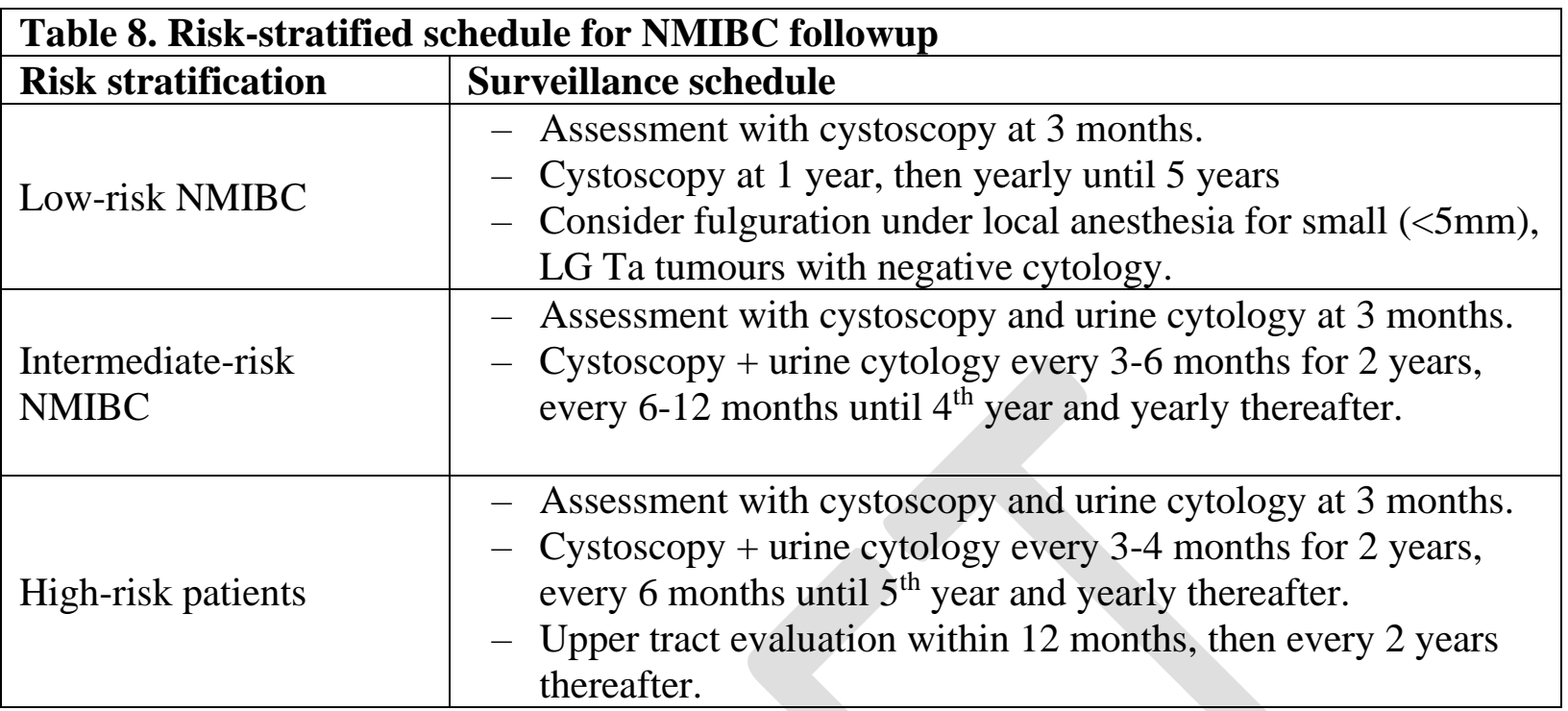

Modified and adapted from Kassouf et $\mathrm{al}^{351}$. LG: low-grade; NMIBC: non-muscle-invasive bladder cancer. 


\section{References}

1. $\quad$ Siegel RL, Miller KD, Jemal A. Cancer statistics, 2019. 2019;69:7-34.

2. Brenner DR, Weir HK, Demers AA, et al. Projected estimates of cancer in Canada in 2020. Canadian Medical Association Journal 2020;192:E199.

3. Demers AA, Brenner DR, Smith L, Shaw A. At-a-glance - Cancer trends in Canada, 1984 to 2015. Health Promot Chronic Dis Prev Can 2019;39:310-4.

4. Dy GW, Gore JL, Forouzanfar MH, Naghavi M, Fitzmaurice C. Global Burden of Urologic Cancers, 1990-2013. European Urology 2017;71:437-46.

5. Amin M, Edge S, Greene F, et al. AJCC Cancer Staging Manual, Springer. 2017.

6. Kulkarni GS, Black PC, Sridhar SS, et al. Canadian Urological Association guideline: Muscle-invasive bladder cancer. Can Urol Assoc J 2019.

7. Warren M, Kolinsky M, Canil CM, et al. Canadian Urological Association/Genitourinary Medical Oncologists of Canada consensus statement: Management of unresectable locally advanced and metastatic urothelial carcinoma. Canadian Urological Association Journal 2019;13:318-27.

8. Avritscher EB, Cooksley CD, Grossman HB, et al. Clinical model of lifetime cost of treating bladder cancer and associated complications. Urology 2006;68:549-53.

9. Kamat AM, Hahn NM, Efstathiou JA, et al. Bladder cancer. Lancet 2016;388:2796-810.

10. Kassouf W, Traboulsi SL, Kulkarni GS, et al. CUA guidelines on the management of non-muscle invasive bladder cancer. Can Urol Assoc J 2015;9:E690-704.

11. Peter Black PG. Bladdder Cancer. A joint SIU-ICUD international consultation.2017.

12. Guyatt GH, Oxman AD, Vist GE, et al. GRADE: an emerging consensus on rating quality of evidence and strength of recommendations. Bmj 2008;336:924-6.

13. Babjuk M, Burger M, Comperat EM, et al. European Association of Urology Guidelines on Non-muscle-invasive Bladder Cancer (TaT1 and Carcinoma In Situ) - 2019 Update. Eur Urol 2019;76:639-57.

14. Chang SS, Boorjian SA, Chou R, et al. Diagnosis and Treatment of Non-Muscle Invasive Bladder Cancer: AUA/SUO Guideline. J Urol 2016;196:1021-9.

15. Bladder cancer: diagnosis and management of bladder cancer: (c) NICE (2015) Bladder cancer: diagnosis and management of bladder cancer. BJU Int 2017;120:755-65.

16. Flaig TW, Spiess PE, Agarwal N, et al. Bladder Cancer, Version 3.2020, NCCN Clinical Practice Guidelines in Oncology. J Natl Compr Canc Netw 2020;18:329-54.

17. Freedman ND, Silverman DT, Hollenbeck AR, Schatzkin A, Abnet CC. Association between smoking and risk of bladder cancer among men and women. JAMA 2011;306:737-45. 18. Burger M, Catto JWF, Dalbagni G, et al. Epidemiology and Risk Factors of Urothelial Bladder Cancer. European Urology 2013;63:234-41. 
19. Cumberbatch MG, Rota M, Catto JWF, La Vecchia C. The Role of Tobacco Smoke in Bladder and Kidney Carcinogenesis: A Comparison of Exposures and Meta-analysis of Incidence and Mortality Risks. European Urology 2016;70:458-66.

20. Baris D, Karagas MR, Verrill C, et al. A case-control study of smoking and bladder cancer risk: emergent patterns over time. Journal of the National Cancer Institute 2009;101:1553-61.

21. Hoover R, Cole P. Population trends in cigarette smoking and bladder cancer. Am J Epidemiol 1971;94:409-18.

22. Crivelli JJ, Xylinas E, Kluth LA, Rieken M, Rink M, Shariat SF. Effect of smoking on outcomes of urothelial carcinoma: a systematic review of the literature. Eur Urol 2014;65:74254.

23. Grotenhuis AJ, Ebben CW, Aben KK, et al. The effect of smoking and timing of smoking cessation on clinical outcome in non-muscle-invasive bladder cancer. Urol Oncol 2015;33:65.e917.

24. Sosnowski R, Przewoźniak K. The role of the urologist in smoking cessation: Why is it important? Urologic Oncology: Seminars and Original Investigations 2015;33:30-9.

25. Fuller TW, Acharya AP, Meyyappan T, et al. Comparison of Bladder Carcinogens in the Urine of E-cigarette Users Versus Non E-cigarette Using Controls. Sci Rep 2018;8:507.

26. Bjurlin MA, Matulewicz RS, Roberts TR, et al. Carcinogen Biomarkers in the Urine of Electronic Cigarette Users and Implications for the Development of Bladder Cancer: A Systematic Review. Eur Urol Oncol 2020.

27. Chacko JA, Heiner JG, Siu W, Macy M, Terris MK. Association between marijuana use and transitional cell carcinoma. Urology 2006;67:100-4.

28. Thomas AA, Wallner LP, Quinn VP, et al. Association between cannabis use and the risk of bladder cancer: results from the California Men's Health Study. Urology 2015;85:388-92.

29. Gandhi S, Vasisth G, Kapoor A. Systematic review of the potential role of cannabinoids as antiproliferative agents for urological cancers. Can Urol Assoc J 2017;11:E138-e42.

30. Rushton L, Bagga S, Bevan R, et al. Occupation and cancer in Britain. Br J Cancer 2010;102:1428-37.

31. Doll R, Peto R. The causes of cancer: quantitative estimates of avoidable risks of cancer in the United States today. J Natl Cancer Inst 1981;66:1191-308.

32. Cumberbatch MG, Cox A, Teare D, Catto JW. Contemporary Occupational Carcinogen Exposure and Bladder Cancer: A Systematic Review and Meta-analysis. JAMA Oncol 2015;1:1282-90.

33. Clayson DB. Specific aromatic amines as occupational bladder carcinogens. Natl Cancer Inst Monogr 1981:15-9. 
34. Droller MJ. Alterations of the p53 gene in occupational bladder cancer in workers exposed to aromatic amines. J Urol 1998;160:618.

35. Schultzel M, Saltzstein SL, Downs TM, Shimasaki S, Sanders C, Sadler GR. Late age (85 years or older) peak incidence of bladder cancer. J Urol 2008;179:1302-5; discussion 5-6.

36. Shariat SF, Milowsky M, Droller MJ. Bladder cancer in the elderly. Urol Oncol 2009;27:653-67.

37. Yancik R, Ries LA. Cancer in older persons: an international issue in an aging world. Semin Oncol 2004;31:128-36.

38. Scosyrev E, Noyes K, Feng C, Messing E. Sex and racial differences in bladder cancer presentation and mortality in the US. Cancer 2009;115:68-74.

39. Palou J, Sylvester RJ, Faba OR, et al. Female gender and carcinoma in situ in the prostatic urethra are prognostic factors for recurrence, progression, and disease-specific mortality in T1G3 bladder cancer patients treated with bacillus Calmette-Guérin. Eur Urol 2012;62:11825.

40. Kluth LA, Rieken M, Xylinas E, et al. Gender-specific differences in clinicopathologic outcomes following radical cystectomy: an international multi-institutional study of more than 8000 patients. Eur Urol 2014;66:913-9.

41. Soave A, Dahlem R, Hansen J, et al. Gender-specific outcomes of bladder cancer patients: a stage-specific analysis in a contemporary, homogenous radical cystectomy cohort. Eur J Surg Oncol 2015;41:368-77.

42. Radkiewicz C, Edgren G, Johansson ALV, et al. Sex Differences in Urothelial Bladder Cancer Survival. Clin Genitourin Cancer 2020;18:26-34.e6.

43. Krimphove MJ, Szymaniak J, Marchese M, et al. Sex-specific Differences in the Quality of Treatment of Muscle-invasive Bladder Cancer Do Not Explain the Overall Survival Discrepancy. Eur Urol Focus 2019.

44. Uhlig A, Strauss A, Seif Amir Hosseini A, et al. Gender-specific Differences in Recurrence of Non-muscle-invasive Bladder Cancer: A Systematic Review and Meta-analysis. Eur Urol Focus 2018;4:924-36.

45. Zhang Y. Understanding the gender disparity in bladder cancer risk: the impact of sex hormones and liver on bladder susceptibility to carcinogens. J Environ Sci Health C Environ Carcinog Ecotoxicol Rev 2013;31:287-304.

46. Kellen E, Hemelt M, Broberg K, et al. Pooled analysis and meta-analysis of the glutathione S-transferase P1 Ile 105Val polymorphism and bladder cancer: a HuGE-GSEC review. Am J Epidemiol 2007;165:1221-30.

47. McGrath M, Michaud DS, De Vivo I. Hormonal and reproductive factors and the risk of bladder cancer in women. Am J Epidemiol 2006;163:236-44. 
48. Koti M, Ingersoll MA, Gupta S, et al. Sex Differences in Bladder Cancer Immunobiology and Outcomes: A Collaborative Review with Implications for Treatment. Eur Urol Oncol 2020;3:622-30.

49. Wallis CJ, Mahar AL, Choo R, et al. Second malignancies after radiotherapy for prostate cancer: systematic review and meta-analysis. Bmj 2016;352:i851.

50. Abern MR, Dude AM, Tsivian M, Coogan CL. The characteristics of bladder cancer after radiotherapy for prostate cancer. Urologic Oncology: Seminars and Original Investigations 2013;31:1628-34.

51. Cumberbatch MGK, Jubber I, Black PC, et al. Epidemiology of Bladder Cancer: A Systematic Review and Contemporary Update of Risk Factors in 2018. European Urology 2018;74:784-95.

52. Pharmaceuticals. Volume 100 A. A review of human carcinogens. IARC Monogr Eval Carcinog Risks Hum 2012;100:1-401.

53. Vlaovic P, Jewett MAS. Cyclophosphamide-induced bladder cancer. Can J Urol 1999;6:745-8.

54. Kostapanos MS, Elisaf MS, Mikhailidis DP. Pioglitazone and cancer: angel or demon? Curr Pharm Des 2013;19:4913-29.

55. Lewis JD, Habel LA, Quesenberry CP, et al. Pioglitazone Use and Risk of Bladder Cancer and Other Common Cancers in Persons With Diabetes. JAMA 2015;314:265-77.

56. Malats N, Real FX. Epidemiology of Bladder Cancer. Hematology/Oncology Clinics of North America 2015;29:177-89.

57. Figueroa JD, Koutros S, Colt JS, et al. Modification of Occupational Exposures on Bladder Cancer Risk by Common Genetic Polymorphisms. Journal of the National Cancer Institute 2015;107:djv223.

58. Lynch HT, de la Chapelle A. Hereditary colorectal cancer. N Engl J Med 2003;348:919-

32.

59. Skeldon SC, Semotiuk K, Aronson M, et al. Patients with Lynch syndrome mismatch repair gene mutations are at higher risk for not only upper tract urothelial cancer but also bladder cancer. Eur Urol 2013;63:379-85.

60. Mork M, Hubosky SG, Roupret M, et al. Lynch Syndrome: A Primer for Urologists and Panel Recommendations. The Journal of urology 2015;194:21-9.

61. Michaud DS. Chronic inflammation and bladder cancer. Urol Oncol 2007;25:260-8.

62. Salem HK, Mahfouz S. Changing patterns (age, incidence, and pathologic types) of schistosoma-associated bladder cancer in Egypt in the past decade. Urology 2012;79:379-83. 63. Francica G, Bellini SA, Scarano F, Miragliuolo A, De Marino FA, Maniscalco M. Correlation of transabdominal sonographic and cystoscopic findings in the diagnosis of focal 
abnormalities of the urinary bladder wall: a prospective study. J Ultrasound Med 2008;27:88794.

64. Datta SN, Allen GM, Evans R, Vaughton KC, Lucas MG. Urinary tract ultrasonography in the evaluation of haematuria--a report of over 1,000 cases. Ann R Coll Surg Engl 2002;84:203-5.

65. Martingano P, Stacul F, Cavallaro M, et al. 64-Slice CT urography: 30 months of clinical experience. Radiol Med 2010;115:920-35.

66. Palou J, Rodríguez-Rubio F, Huguet J, et al. Multivariate analysis of clinical parameters of synchronous primary superficial bladder cancer and upper urinary tract tumor. J Urol 2005;174:859-61; discussion 61.

67. Hilton S, Jones LP. Recent advances in imaging cancer of the kidney and urinary tract. Surg Oncol Clin N Am 2014;23:863-910.

68. Panebianco V, Narumi Y, Altun E, et al. Multiparametric Magnetic Resonance Imaging for Bladder Cancer: Development of VI-RADS (Vesical Imaging-Reporting And Data System). European Urology 2018;74:294-306.

69. Woo S, Panebianco V, Narumi Y, et al. Diagnostic Performance of Vesical Imaging Reporting and Data System for the Prediction of Muscle-invasive Bladder Cancer: A Systematic Review and Meta-analysis. Eur Urol Oncol 2020;3:306-15.

70. Krajewski W, Kościelska-Kasprzak K, Rymaszewska J, Zdrojowy R. How different cystoscopy methods influence patient sexual satisfaction, anxiety, and depression levels: a randomized prospective trial. Qual Life Res 2017;26:625-34.

71. Berajoui MB, Aditya I, Herrera-Caceres J, et al. A Prospective Randomized Controlled Trial of Irrigation "Bag Squeeze" to Manage Pain for Patients Undergoing Flexible Cystoscopy. J Urol 2020;204:1012-8.

72. Casteleijn NF, Vriesema JL, Stomps SP, van Balen OL, Cornel EB. The effect of office based flexible and rigid cystoscopy on pain experience in female patients. Investig Clin Urol 2017;58:48-53.

73. Patel AR, Jones JS, Babineau D. Lidocaine $2 \%$ gel versus plain lubricating gel for pain reduction during flexible cystoscopy: a meta-analysis of prospective, randomized, controlled trials. J Urol 2008;179:986-90.

74. Aaronson DS, Walsh TJ, Smith JF, Davies BJ, Hsieh MH, Konety BR. Meta-analysis: does lidocaine gel before flexible cystoscopy provide pain relief? BJU Int 2009;104:506-9; discussion 9-10.

75. Amling CL. Diagnosis and management of superficial bladder cancer. Current Problems in Cancer 2001;25:IN1-278.

76. Keller AK, Jensen JB. Voided urine versus bladder washing cytology for detection of urothelial carcinoma: which is better? Scand J Urol 2017;51:290-2. 
77. Ramakumar S, Bhuiyan J, Besse JA, et al. COMPARISON OF SCREENING METHODS IN THE DETECTION OF BLADDER CANCER. The Journal of Urology 1999;161:388-94.

78. Brown FM. Urine cytology. It is still the gold standard for screening? Urol Clin North Am 2000;27:25-37.

79. Raitanen M-P, Aine R, Rintala E, et al. Differences between local and review urinary cytology in diagnosis of bladder cancer. An interobserver multicenter analysis. European urology 2002;41:284-9.

80. Yafi FA, Brimo F, Steinberg J, Aprikian AG, Tanguay S, Kassouf W. Prospective analysis of sensitivity and specificity of urinary cytology and other urinary biomarkers for bladder cancer. Urologic Oncology: Seminars and Original Investigations 2015;33:66.e2566.e31.

81. Konety BR, Getzenberg RH. URINE BASED MARKERS OF UROLOGICAL MALIGNANCY. The Journal of Urology 2001;165:600-11.

82. Heney NM. Natural history of superficial bladder cancer. Prognostic features and longterm disease course. Urol Clin North Am 1992;19:429-33.

83. Sylvester RJ, van der Meijden APM, Oosterlinck W, et al. Predicting Recurrence and Progression in Individual Patients with Stage Ta T1 Bladder Cancer Using EORTC Risk Tables: A Combined Analysis of 2596 Patients from Seven EORTC Trials. European Urology 2006;49:466-77.

84. Millan-Rodriguez F, Chechile-Toniolo G, Salvador-Bayarri J, Palou J, VicenteRodriguez J. Multivariate analysis of the prognostic factors of primary superficial bladder cancer. The Journal of urology 2000;163:73-8.

85. MacLennan GT, Kirkali Z, Cheng L. Histologic grading of noninvasive papillary urothelial neoplasms. Eur Urol 2007;51:889-97; discussion 97-8.

86. Soukup V, Čapoun O, Cohen D, et al. Prognostic Performance and Reproducibility of the 1973 and 2004/2016 World Health Organization Grading Classification Systems in Non-muscleinvasive Bladder Cancer: A European Association of Urology Non-muscle Invasive Bladder Cancer Guidelines Panel Systematic Review. European Urology 2017;72:801-13.

87. Kirkali Z, Chan T, Manoharan M, et al. Bladder cancer: Epidemiology, staging and grading, and diagnosis. Urology 2005;66:4-34.

88. Sylvester RJ, van der Meijden A, Witjes JA, et al. High-grade Ta urothelial carcinoma and carcinoma in situ of the bladder. Urology 2005;66:90-107.

89. Matulay JT, Soloway M, Witjes JA, et al. Risk-adapted management of low-grade bladder tumours: recommendations from the International Bladder Cancer Group (IBCG). BJU International 2020;125:497-505. 
90. Norming U, Tribukait B, Nyman CR, Nilsson B, Wang N. Prognostic significance of mucosal aneuploidy in stage Ta/T1 grade 3 carcinoma of the bladder. J Urol 1992;148:1420-6; discussion 6-7.

91. Quhal F, D'Andrea D, Soria F, et al. Primary Ta high grade bladder tumors: Determination of the risk of progression. Urol Oncol 2020.

92. Martin-Doyle W, Leow JJ, Orsola A, Chang SL, Bellmunt J. Improving selection criteria for early cystectomy in high-grade t1 bladder cancer: a meta-analysis of 15,215 patients. J Clin Oncol 2015;33:643-50.

93. Cao D, Vollmer RT, Luly J, et al. Comparison of 2004 and 1973 World Health Organization Grading Systems and Their Relationship to Pathologic Staging for Predicting Long-term Prognosis in Patients With Urothelial Carcinoma. Urology 2010;76:593-9.

94. Otto W, Denzinger S, Fritsche H-M, et al. The WHO classification of 1973 is more suitable than the WHO classification of 2004 for predicting survival in pT1 urothelial bladder cancer. BJU International 2011;107:404-8.

95. Amin MB, Smith SC, Reuter VE, et al. Update for the practicing pathologist: The International Consultation On Urologic Disease-European association of urology consultation on bladder cancer. Mod Pathol 2015;28:612-30.

96. Subiela JD, Palou J, Esquinas C, Fernandez Gomez JM, Rodriguez Faba O. Clinical usefulness of random biopsies in diagnosis and treatment of non-muscle invasive bladder cancer: Systematic review and meta-analysis. Actas Urol Esp 2018;42:285-98.

97. Knowles MA, Hurst CD. Molecular biology of bladder cancer: new insights into pathogenesis and clinical diversity. Nat Rev Cancer 2015;15:25-41.

98. Lamm DL. Carcinoma in situ. Urol Clin North Am 1992;19:499-508.

99. Shariat SF, Palapattu GS, Amiel GE, et al. Characteristics and outcomes of patients with carcinoma in situ only at radical cystectomy. Urology 2006;68:538-42.

100. Hudson MLA, Herr HW. Carcinoma in Situ of the Bladder. The Journal of Urology 1995;153:564-72.

101. Akhtar M, Al-Bozom IA, Ben Gashir M, Taha NM, Rashid S, Al-Nabet A. Urothelial Carcinoma In Situ (CIS): New Insights. Adv Anat Pathol 2019;26:313-9.

102. Takenaka A, Yamada Y, Miyake H, Hara I, Fujisawa M. Clinical outcomes of bacillus Calmette-Guerin instillation therapy for carcinoma in situ of urinary bladder. Int J Urol 2008;15:309-13.

103. Melicow MM, Hollowell JW. Intra-urothelial cancer: carcinoma in situ, Bowen's disease of the urinary system: discussion of thirty cases. J Urol 1952;68:763-72.

104. Miyake M, Gotoh D, Shimada K, et al. Exploration of risk factors predicting outcomes for primary T1 high-grade bladder cancer and validation of the Spanish Urological Club for 
Oncological Treatment scoring model: Long-term followup experience at a single institute. Int J Urol 2015;22:541-7.

105. Brimo F, Wu C, Zeizafoun N, et al. Prognostic factors in T1 bladder urothelial carcinoma: the value of recording millimetric depth of invasion, diameter of invasive carcinoma, and muscularis mucosa invasion. Hum Pathol 2013;44:95-102.

106. Mathieu R, Lucca I, Roupret M, Briganti A, Shariat SF. The prognostic role of lymphovascular invasion in urothelial carcinoma of the bladder. Nat Rev Urol 2016;13:471-9. 107. Cho KS, Seo HK, Joung JY, et al. Lymphovascular invasion in transurethral resection specimens as predictor of progression and metastasis in patients with newly diagnosed $\mathrm{T} 1$ bladder urothelial cancer. J Urol 2009;182:2625-30.

108. Kim HS, Kim M, Jeong CW, Kwak C, Kim HH, Ku JH. Presence of lymphovascular invasion in urothelial bladder cancer specimens after transurethral resections correlates with risk of upstaging and survival: a systematic review and meta-analysis. Urol Oncol 2014;32:1191-9. 109. Mari A, Kimura S, Foerster B, et al. A systematic review and meta-analysis of the impact of lymphovascular invasion in bladder cancer transurethral resection specimens. BJU Int 2019;123:11-21.

110. Algaba F. Lymphovascular invasion as a prognostic tool for advanced bladder cancer. Curr Opin Urol 2006;16:367-71.

111. van Rhijn BW, Liu L, Vis AN, et al. Prognostic value of molecular markers, sub-stage and European Organisation for the Research and Treatment of Cancer risk scores in primary T1 bladder cancer. BJU Int 2012;110:1169-76.

112. van Rhijn BW, van der Kwast TH, Alkhateeb SS, et al. A new and highly prognostic system to discern T1 bladder cancer substage. Eur Urol 2012;61:378-84.

113. Fransen van de Putte EE, Otto W, Hartmann A, et al. Metric substage according to micro and extensive lamina propria invasion improves prognostics in T1 bladder cancer. Urol Oncol 2018;36:361.e7-.e13.

114. Kardoust Parizi M, Enikeev D, Glybochko PV, et al. Prognostic value of T1 substaging on oncological outcomes in patients with non-muscle-invasive bladder urothelial carcinoma: a systematic literature review and meta-analysis. World J Urol 2020;38:1437-49.

115. Orsola A, Cecchini L, Raventós CX, et al. Risk factors for positive findings in patients with high-grade T1 bladder cancer treated with transurethral resection of bladder tumour (TUR) and bacille Calmette-Guérin therapy and the decision for a repeat TUR. BJU Int 2010;105:202-7. 116. Orsola A, Werner L, de Torres I, et al. Reexamining treatment of high-grade T1 bladder cancer according to depth of lamina propria invasion: a prospective trial of 200 patients. $\mathrm{Br} \mathrm{J}$ Cancer 2015;112:468-74. 
117. Soukup V, Babjuk M, Dusková J, et al. Does the expression of fascin-1 and tumor subclassification help to assess the risk of recurrence and progression in t1 urothelial urinary bladder carcinoma? Urol Int 2008;80:413-8.

118. Parmar MK, Freedman LS, Hargreave TB, Tolley DA. Prognostic factors for recurrence and followup policies in the treatment of superficial bladder cancer: report from the British Medical Research Council Subgroup on Superficial Bladder Cancer (Urological Cancer Working Party). J Urol 1989;142:284-8.

119. Loening S, Narayana A, Yoder L, Slymen D, Penick G, Culp D. Analysis of bladder tumor recurrence in 178 patients. Urology 1980;16:137-41.

120. Mulders PF, Meyden AP, Doesburg WH, Oosterhof GO, Debruyne FM. Prognostic factors in pTa-pT1 superficial bladder tumours treated with intravesical instillations. The Dutch South-Eastern Urological Collaborative Group. Br J Urol 1994;73:403-8.

121. Shinka T, Matsumoto M, Ogura H, Hirano A, Ohkawa T. Recurrence of primary superficial bladder cancer treated with prophylactic intravesical Tokyo 172 bacillus CalmetteGuerin: a long-term followup. Int J Urol 1997;4:139-43.

122. Fernandez-Gomez J, Madero R, Solsona E, et al. Predicting nonmuscle invasive bladder cancer recurrence and progression in patients treated with bacillus Calmette-Guerin: the CUETO scoring model. J Urol 2009;182:2195-203.

123. Sylvester RJ, Rodríguez O, Hernández V, et al. European Association of Urology (EAU) Prognostic Factor Risk Groups for Non-muscle-invasive Bladder Cancer (NMIBC) Incorporating the WHO 2004/2016 and WHO 1973 Classification Systems for Grade: An Update from the EAU NMIBC Guidelines Panel. Eur Urol 2021.

124. Dalesio O, Schulman CC, Sylvester R, et al. Prognostic factors in superficial bladder tumors. A study of the European Organization for Research on Treatment of Cancer:

Genitourinary Tract Cancer Cooperative Group. J Urol 1983;129:730-3.

125. Brausi M, Collette L, Kurth K, et al. Variability in the recurrence rate at first followup cystoscopy after TUR in stage Ta T1 transitional cell carcinoma of the bladder: a combined analysis of seven EORTC studies. Eur Urol 2002;41:523-31.

126. Cambier S, Sylvester RJ, Collette L, et al. EORTC Nomograms and Risk Groups for Predicting Recurrence, Progression, and Disease-specific and Overall Survival in Non-Muscleinvasive Stage Ta-T1 Urothelial Bladder Cancer Patients Treated with 1-3 Years of Maintenance Bacillus Calmette-Guerin. Eur Urol 2016;69:60-9.

127. Lobo N, Shariat SF, Guo CC, et al. What Is the Significance of Variant Histology in Urothelial Carcinoma? European Urology Focus 2019.

128. Shah RB, Montgomery JS, Montie JE, Kunju LP. Variant (divergent) histologic differentiation in urothelial carcinoma is under-recognized in community practice: Impact of 
mandatory central pathology review at a large referral hospital. Urologic Oncology: Seminars and Original Investigations 2013;31:1650-5.

129. Abd El-Latif A, Watts KE, Elson P, Fergany A, Hansel DE. The sensitivity of initial transurethral resection or biopsy of bladder tumor(s) for detecting bladder cancer variants on radical cystectomy. J Urol 2013;189:1263-7.

130. Wasco MJ, Daignault S, Zhang Y, et al. Urothelial carcinoma with divergent histologic differentiation (mixed histologic features) predicts the presence of locally advanced bladder cancer when detected at transurethral resection. Urology 2007;70:69-74.

131. Izard JP, Siemens DR, Mackillop WJ, et al. Outcomes of squamous histology in bladder cancer: a population-based study. Urol Oncol 2015;33:425.e7-13.

132. Mally AD, Tin AL, Lee JK, et al. Clinical Outcomes of Patients With T1 Nested Variant of Urothelial Carcinoma Compared to Pure Urothelial Carcinoma of the Bladder. Clin Genitourin Cancer 2017.

133. Linder BJ, Frank I, Cheville JC, et al. Outcomes following radical cystectomy for nested variant of urothelial carcinoma: a matched cohort analysis. J Urol 2013;189:1670-5.

134. Seisen T, Comperat E, Leon P, Roupret M. Impact of histological variants on the outcomes of nonmuscle invasive bladder cancer after transurethral resection. Curr Opin Urol 2014;24:524-31.

135. Porten SP, Willis D, Kamat AM. Variant histology: role in management and prognosis of nonmuscle invasive bladder cancer. Curr Opin Urol 2014;24:517-23.

136. Mariappan P, Finney SM, Head E, et al. Good quality white-light transurethral resection of bladder tumours (GQ-WLTURBT) with experienced surgeons performing complete resections and obtaining detrusor muscle reduces early recurrence in new non-muscle-invasive bladder cancer: validation across time and place and recommendation for benchmarking. BJU Int 2012;109:1666-73.

137. Herr HW, Donat SM. Quality control in transurethral resection of bladder tumours. BJU International 2008;102:1242-6.

138. Anderson C, Weber R, Patel D, et al. A 10-Item Checklist Improves Reporting of Critical Procedural Elements during Transurethral Resection of Bladder Tumor. J Urol 2016;196:101420.

139. Suarez-Ibarrola R, Soria F, Abufaraj M, et al. Surgical checklist impact on recurrencefree survival of patients with non-muscle-invasive bladder cancer undergoing transurethral resection of bladder tumour. BJU Int 2019;123:646-50.

140. Panagoda PI, Vasdev N, Gowrie-Mohan S. Avoiding the Obturator Jerk during TURBT. Current Urology 2018;12:1-5. 
141. Koumpan Y, Jaeger M, Mizubuti GB, et al. Spinal Anesthesia is Associated with Lower Recurrence Rates after Resection of Nonmuscle Invasive Bladder Cancer. The Journal of Urology 2018;199:940-6.

142. Zhao C, Tang K, Yang H, Xia D, Chen Z. Bipolar Versus Monopolar Transurethral Resection of Nonmuscle-Invasive Bladder Cancer: A Meta-Analysis. J Endourol 2016;30:5-12. 143. Sharma G, Sharma AP, Mavuduru RS, et al. Safety and efficacy of bipolar versus monopolar transurethral resection of bladder tumor: a systematic review and meta-analysis. World Journal of Urology 2020.

144. Pearce S, Daneshmand S. Enhanced Endoscopy in Bladder Cancer. Current Urology Reports 2018;19:84.

145. Zaak D, Karl A, Knuchel R, et al. Diagnosis of urothelial carcinoma of the bladder using fluorescence endoscopy. BJU Int 2005;96:217-22.

146. Fradet Y, Grossman HB, Gomella L, et al. A comparison of hexaminolevulinate fluorescence cystoscopy and white light cystoscopy for the detection of carcinoma in situ in patients with bladder cancer: a phase III, multicenter study. J Urol 2007;178:68-73; discussion 147. Filbeck T, Pichlmeier U, Knuechel R, Wieland WF, Roessler W. Clinically relevant improvement of recurrence-free survival with 5-aminolevulinic acid induced fluorescence diagnosis in patients with superficial bladder tumors. J Urol 2002;168:67-71.

148. Sfetsas K, Mitropoulos D. Reducing understaging of bladder cancer with the aid of photodynamic cystoscopy. J Egypt Natl Canc Inst 2016;28:89-94.

149. Stenzl A, Burger M, Fradet Y, et al. Hexaminolevulinate Guided Fluorescence Cystoscopy Reduces Recurrence in Patients With Nonmuscle Invasive Bladder Cancer. The Journal of Urology 2010;184:1907-14.

150. Burger M, Grossman HB, Droller M, et al. Photodynamic diagnosis of non-muscleinvasive bladder cancer with hexaminolevulinate cystoscopy: a meta-analysis of detection and recurrence based on raw data. Eur Urol 2013;64:846-54.

151. Grossman HB, Stenzl A, Fradet Y, et al. Long-term decrease in bladder cancer recurrence with hexaminolevulinate enabled fluorescence cystoscopy. J Urol 2012;188:58-62.

152. Geavlete B, Multescu R, Georgescu D, Jecu M, Stanescu F, Geavlete P. Treatment changes and long-term recurrence rates after hexaminolevulinate (HAL) fluorescence cystoscopy: does it really make a difference in patients with non-muscle-invasive bladder cancer (NMIBC)? BJU Int 2012;109:549-56.

153. Gallagher KM, Gray K, Anderson $\mathrm{CH}$, et al. 'Real-life experience': recurrence rate at 3 years with Hexvix(®) photodynamic diagnosis-assisted TURBT compared with good quality white light TURBT in new NMIBC-a prospective controlled study. World J Urol 2017;35:18717. 
154. Gakis G, Fahmy O. Systematic Review and Meta-Analysis on the Impact of Hexaminolevulinate- Versus White-Light Guided Transurethral Bladder Tumor Resection on Progression in Non-Muscle Invasive Bladder Cancer. Bladder Cancer 2016;2:293-300. 155. O'Brien T, Ray E, Chatterton K, Khan MS, Chandra A, Thomas K. Prospective randomized trial of hexylaminolevulinate photodynamic-assisted transurethral resection of bladder tumour (TURBT) plus single-shot intravesical mitomycin C vs conventional white-light TURBT plus mitomycin $\mathrm{C}$ in newly presenting non-muscle-invasive bladder cancer. BJU Int 2013;112:1096-104.

156. Svatek RS, Hollenbeck BK, Holmang S, et al. The economics of bladder cancer: costs and considerations of caring for this disease. Eur Urol 2014;66:253-62.

157. Sievert KD, Amend B, Nagele U, et al. Economic aspects of bladder cancer: what are the benefits and costs? World J Urol 2009;27:295-300.

158. Witjes JA, Babjuk M, Gontero P, et al. Clinical and cost effectiveness of hexaminolevulinate-guided blue-light cystoscopy: evidence review and updated expert recommendations. Eur Urol 2014;66:863-71.

159. Klaassen Z, Li K, Kassouf W, Black PC, Dragomir A, Kulkarni GS. Contemporary costconsequence analysis of blue light cystoscopy with hexaminolevulinate in non-muscle-invasive bladder cancer. Can Urol Assoc J 2017;11:173-81.

160. Bryan RT, Billingham LJ, Wallace DMA. Narrow-band imaging flexible cystoscopy in the detection of recurrent urothelial cancer of the bladder. BJU international 2008;101:702-6. 161. Herr HW, Donat SM. Reduced bladder tumour recurrence rate associated with narrowband imaging surveillance cystoscopy. BJU Int 2011;107:396-8.

162. Cauberg EC, Mamoulakis C, de la Rosette JJ, de Reijke TM. Narrow band imagingassisted transurethral resection for non-muscle invasive bladder cancer significantly reduces residual tumour rate. World J Urol 2011;29:503-9.

163. Naselli A, Hurle R, Puppo P. The role of narrow-band imaging in the management of non-muscle-invasive bladder cancer. Expert Rev Anticancer Ther 2012;12:1523-8.

164. Geavlete BF, Brînzea A, ChecheriŢă IA, et al. Carcinoma in situ of the urinary bladder from pathology to narrow band imaging. Rom J Morphol Embryol 2015;56:1069-76.

165. Naito S, Algaba F, Babjuk M, et al. The Clinical Research Office of the Endourological Society (CROES) Multicentre Randomised Trial of Narrow Band Imaging\&\#x2013;Assisted Transurethral Resection of Bladder Tumour (TURBT) Versus Conventional White Light Imaging\&\#x2013;Assisted TURBT in Primary Non\&\#x2013; Muscle-invasive Bladder Cancer Patients: Trial Protocol and 1-year Results. European Urology 2016;70:506-15.

166. Kang W, Cui Z, Chen Q, Zhang D, Zhang H, Jin X. Narrow band imaging-assisted transurethral resection reduces the recurrence risk of non-muscle invasive bladder cancer: A systematic review and meta-analysis. Oncotarget 2017;8:23880-90. 
167. Ukai R, Kawashita E, Ikeda H. A new technique for transurethral resection of superficial bladder tumor in 1 piece. J Urol 2000;163:878-9.

168. Kawada T, Ebihara K, Suzuki T, Imai K, Yamanaka H. A new technique for transurethral resection of bladder tumors: rotational tumor resection using a new arched electrode. J Urol 1997; 157:2225-6.

169. Bach T, Muschter R, Herrmann TR, et al. Technical solutions to improve the management of non-muscle-invasive transitional cell carcinoma: summary of a European Association of Urology Section for Uro-Technology (ESUT) and Section for Uro-Oncology (ESOU) expert meeting and current and future perspectives. BJU Int 2015;115:14-23.

170. Hashem A, Mosbah A, El-Tabey NA, et al. Holmium Laser En-bloc Resection Versus Conventional Transurethral Resection of Bladder Tumors for Treatment of Non-muscle-invasive Bladder Cancer: A Randomized Clinical Trial. Eur Urol Focus 2020.

171. Gakis G, Karl A, Bertz S, et al. Transurethral en bloc submucosal hydrodissection vs conventional resection for resection of non-muscle-invasive bladder cancer (HYBRIDBLUE): a randomised, multicentre trial. BJU Int 2020;126:509-19.

172. Yanagisawa T, Yorozu T, Miki J, et al. Feasibility and accuracy of pathological diagnosis in en bloc resection versus conventional transurethral resection of bladder tumor: Evaluation with pT1 sub-staging by 10 pathologists. Histopathology 2020.

173. Teoh JY, MacLennan S, Chan VW, et al. An International Collaborative Consensus Statement on En Bloc Resection of Bladder Tumour Incorporating Two Systematic Reviews, a Two-round Delphi Survey, and a Consensus Meeting. Eur Urol 2020.

174. Mori K, D'Andrea D, Enikeev DV, Egawa S, Shariat SF. En bloc resection for nonmuscle invasive bladder cancer: review of the recent literature. Curr Opin Urol 2020;30:41-7.

175. Herr HW. The value of a second transurethral resection in evaluating patients with bladder tumors. J Urol 1999;162:74-6.

176. Ali MH, Ismail IY, Eltobgy A, Gobeish A. Evaluation of second-look transurethral resection in restaging of patients with nonmuscle-invasive bladder cancer. J Endourol 2010;24:2047-50.

177. Bishr M, Lattouf JB, Latour M, Saad F. Tumour stage on re-staging transurethral resection predicts recurrence and progression-free survival of patients with high-risk non-muscle invasive bladder cancer. Can Urol Assoc J 2014;8:E306-10.

178. Divrik RT, Sahin AF, Yildirim U, Altok M, Zorlu F. Impact of routine second transurethral resection on the long-term outcome of patients with newly diagnosed pT1 urothelial carcinoma with respect to recurrence, progression rate, and disease-specific survival: a prospective randomised clinical trial. Eur Urol 2010;58:185-90.

179. Cumberbatch MGK, Foerster B, Catto JWF, et al. Repeat Transurethral Resection in Non-muscle-invasive Bladder Cancer: A Systematic Review. Eur Urol 2018;73:925-33. 
180. Naselli A, Hurle R, Paparella S, et al. Role of Restaging Transurethral Resection for T1 Non-muscle invasive Bladder Cancer: A Systematic Review and Meta-analysis. European urology focus 2018;4:558-67.

181. Herr HW. RESTAGING TRANSURETHRAL RESECTION OF HIGH-RISK SUPERFICIAL BLADDER CANCER IMPROVES THE INITIAL RESPONSE TO BACILLUS CALMETTE-GUERIN THERAPY. The Journal of Urology 2005;174:2134-7. 182. Yuk HD, Jeong CW, Kwak C, Kim HH, Ku JH. Should intravesical Bacillus CalmetteGuerin (BCG) treatment be administered to patients with $\mathrm{T} 0$ after repeat transurethral resection of bladder tumor in patients with high-risk non-muscle invasive bladder cancer? PLoS One 2018;13:e0208267.

183. Baltaci S, Bozlu M, Yildirim A, et al. Significance of the interval between first and second transurethral resection on recurrence and progression rates in patients with high-risk nonmuscle-invasive bladder cancer treated with maintenance intravesical Bacillus Calmette-Guerin. BJU Int 2015;116:721-6.

184. Krajewski W, Zdrojowy R, Dembowski J, et al. The Optimal Timing of Restaging Resection before Introduction of Bacillus Calmette-Guerin Immunotherapy in Patients with High-Risk Non-Muscle-Invasive Bladder Cancer. Urol Int 2019;102:60-8.

185. Czech AK, Gronostaj K, Frydrych J, et al. Identification of potential prognostic factors for absence of residual disease in the second resection of $\mathrm{T} 1$ bladder cancer. Cent European $\mathrm{J}$ Urol 2019;72:252-7.

186. Gill TS, Das RK, Basu S, Dey RK, Mitra S. Predictive factors for residual tumor and tumor upstaging on relook transurethral resection of bladder tumor in non-muscle invasive bladder cancer. Urol Ann 2014;6:305-8.

187. Ayati M, Amini E, Shahrokhi Damavand R, et al. Second Transurethral Resection of Bladder Tumor: Is it Necessary in All T1 and/or High-Grade Tumors? Urol J 2019;16:152-6. 188. Dangi AD, Kumar RM, Kodiatte TA, et al. Is there a role for second transurethral resection in pTa high-grade urothelial bladder cancer? Cent European J Urol 2018;71:287-94. 189. Gontero P, Sylvester R, Pisano F, et al. The impact of re-transurethral resection on clinical outcomes in a large multicentre cohort of patients with T1 high-grade/Grade 3 bladder cancer treated with bacille Calmette-Guérin. BJU Int 2016;118:44-52.

190. Taoka R, Matsuoka Y, Kohashiguchi K, et al. Impact of second transurethral resection on recurrence in patients with high-grade Ta bladder cancer. Int J Urol 2020;27:1130-5.

191. Mastroianni R, Brassetti A, Krajewski W, et al. Assessing the Impact of the Absence of Detrusor Muscle in Ta Low-grade Urothelial Carcinoma of the Bladder on Recurrence-free Survival. Eur Urol Focus 2020.

192. van der Meijden A, Oosterlinck W, Brausi M, Kurth KH, Sylvester R, de Balincourt C. Significance of bladder biopsies in Ta,T1 bladder tumors: a report from the EORTC Genito- 
Urinary Tract Cancer Cooperative Group. EORTC-GU Group Superficial Bladder Committee. European urology 1999;35:267-71.

193. Hara T, Takahashi M, Gondo T, et al. Risk of concomitant carcinoma in situ determining biopsy candidates among primary non-muscle-invasive bladder cancer patients: retrospective analysis of 173 Japanese cases. Int J Urol 2009;16:293-8.

194. Lerner SP, Shen S. Pathologic assessment and clinical significance of prostatic involvement by transitional cell carcinoma and prostate cancer. Urol Oncol 2008;26:481-5. 195. Walsh DL, Chang SS. Dilemmas in the treatment of urothelial cancers of the prostate. Urologic Oncology: Seminars and Original Investigations 2009;27:352-7.

196. Herr HW. Extravesical tumor relapse in patients with superficial bladder tumors. Journal of Clinical Oncology 1998;16:1099-102.

197. Grabstald H. Prostatic Biopsy in selected Patients with Carcinoma in Situ of the Bladder: Preliminary Report. The Journal of Urology 1984;132:1117-8.

198. Bretton PR, Herr HW, Whitmore WF, et al. Intravesical Bacillus Calmette-Guerin Therapy for in situ Transitional Cell Carcinoma Involving the Prostatic Urethra. The Journal of Urology 1989;141:853-5.

199. Palou J, Sylvester RJ, Faba OR, et al. Female gender and carcinoma in situ in the prostatic urethra are prognostic factors for recurrence, progression, and disease-specific mortality in T1G3 bladder cancer patients treated with bacillus Calmette-Guerin. Eur Urol 2012;62:11825.

200. Mungan MU, Canda AE, Tuzel E, Yorukoglu K, Kirkali Z. Risk factors for mucosal prostatic urethral involvement in superficial transitional cell carcinoma of the bladder. Eur Urol 2005;48:760-3.

201. Brant A, Daniels M, Chappidi MR, et al. Prognostic implications of prostatic urethral involvement in non-muscle-invasive bladder cancer. World J Urol 2019;37:2683-9.

202. Solsona E, Iborra I, Ricos JV, Monros JL, Casanova JL, Almenar S. The Prostate Involvement As Prognostic Factor in Patients with Superficial Bladder Tumors. The Journal of Urology 1995; 154:1710-3.

203. Sakamoto N, Tsuneyoshi M, Naito S, Kumazawa J. An adequate sampling of the prostate to identify prostatic involvement by urothelial carcinoma in bladder cancer patients. J Urol 1993;149:318-21.

204. Palou J, Baniel J, Klotz L, et al. Urothelial carcinoma of the prostate. Urology 2007;69:50-61.

205. Orihuela E, Herr HW, Whitmore WF. Conservative treatment of superficial transitional cell carcinoma of prostatic urethra with intravesical BCG. Urology 1989;34:231-7. 
206. Palou Redorta J, Schatteman P, Huguet Pérez J, et al. Intravesical instillations with bacillus calmette-guérin for the treatment of carcinoma in situ involving prostatic ducts. Eur Urol 2006;49:834-8; discussion 8.

207. Wishnow KI, Ro JY. Importance of early treatment of transitional cell carcinoma of prostatic ducts. Urology 1988;32:11-2.

208. Brausi M, Collette L, Kurth K, et al. Variability in the Recurrence Rate at First Followup Cystoscopy after TUR in Stage Ta T1 Transitional Cell Carcinoma of the Bladder: A Combined Analysis of Seven EORTC Studies. European Urology 2002;41:523-31.

209. Pan JS, Slocum HK, Rustum YM, Greco WR, Gaeta JF, Huben RP. Inhibition of implantation of murine bladder tumor by thiotepa in cauterized bladder. J Urol 1989;142:158993.

210. Tolley DA, Parmar MK, Grigor KM, et al. The effect of intravesical mitomycin C on recurrence of newly diagnosed superficial bladder cancer: a further report with 7 years of follow up. The Journal of urology 1996;155:1233-8.

211. Solsona E, Iborra I, Ricos JV, Monros JL, Casanova J, Dumont R. Effectiveness of a single immediate mitomycin $\mathrm{C}$ instillation in patients with low-risk superficial bladder cancer: short and long-term followup. J Urol 1999;161:1120-3.

212. De Nunzio C, Carbone A, Albisinni S, et al. Long-term experience with early single mitomycin $\mathrm{C}$ instillations in patients with low-risk non-muscle-invasive bladder cancer: prospective, single-centre randomised trial. World J Urol 2011;29:517-21.

213. Barghi MR, Rahmani MR, Hosseini Moghaddam SM, Jahanbin M. Immediate intravesical instillation of mitomycin $\mathrm{C}$ after transurethral resection of bladder tumor in patients with low-risk superficial transitional cell carcinoma of bladder. Urol J 2006;3:220-4.

214. El-Ghobashy S, El-Leithy TR, Roshdy MM, El-Ganzoury HM. Effectiveness of a single immediate mitomycin $\mathrm{C}$ instillation in patients with low-risk superficial bladder cancer: short and long-term followup. J Egypt Natl Canc Inst 2007;19:121-6.

215. Oosterlinck W, Kurth KH, Schroder F, Bultinck J, Hammond B, Sylvester R. A prospective European Organization for Research and Treatment of Cancer Genitourinary Group randomized trial comparing transurethral resection followed by a single intravesical instillation of epirubicin or water in single stage Ta, T1 papillary carcinoma of the bladder. J Urol 1993;149:749-52.

216. Ali-el-Dein B, Nabeeh A, el-Baz M, Shamaa S, Ashamallah A. Single-dose versus multiple instillations of epirubicin as prophylaxis for recurrence after transurethral resection of pTa and pT1 transitional-cell bladder tumours: a prospective, randomized controlled study. Br J Urol 1997;79:731-5.

217. Rajala P, Kaasinen E, Raitanen M, Liukkonen T, Rintala E. Perioperative Single Dose Instillation of Epirubicin or Interferon- $\alpha$ After Transurethral Resection for The Prophylaxis of 
Primary Superficial Bladder Cancer Recurrence: A Prospective Randomized Multicenter Study - Finnbladder III Long-Term Results. The Journal of Urology 2002;168:981-5.

218. Berrum-Svennung I, Granfors T, Jahnson S, Boman H, Holmang S. A single instillation of epirubicin after transurethral resection of bladder tumors prevents only small recurrences. J Urol 2008;179:101-5; discussion 5-6.

219. Okamura K, Ono Y, Kinukawa T, et al. Randomized study of single early instillation of (2"R)-4'-O-tetrahydropyranyl-doxorubicin for a single superficial bladder carcinoma. Cancer 2002;94:2363-8.

220. Miki T, Nonomura N, Kojima Y, et al. [A randomized study on intravesical pirarubicin (THP) chemoprophylaxis of recurrence after transurethral resection of superficial bladder cancer]. Hinyokika Kiyo 1997;43:907-12.

221. The effect of intravesical thiotepa on tumour recurrence after endoscopic treatment of newly diagnosed superficial bladder cancer. A further report with long-term followup of a Medical Research Council randomized trial. Medical Research Council Working Party on Urological Cancer, Subgroup on Superficial Bladder Cancer. Br J Urol 1994;73:632-8. 222. Messing EM, Tangen CM, Lerner SP, et al. Effect of Intravesical Instillation of Gemcitabine vs Saline Immediately Following Resection of Suspected Low-Grade Non-MuscleInvasive Bladder Cancer on Tumor Recurrence: SWOG S0337 Randomized Clinical Trial. JAMA 2018;319:1880-8.

223. Gudjónsson S, Adell L, Merdasa F, et al. Should All Patients with Non-Muscle-Invasive Bladder Cancer Receive Early Intravesical Chemotherapy after Transurethral Resection? The Results of a Prospective Randomised Multicentre Study. European Urology 2009;55:773-80. 224. Sylvester RJ, Oosterlinck W, van der Meijden AP. A single immediate postoperative instillation of chemotherapy decreases the risk of recurrence in patients with stage Ta T1 bladder cancer: a meta-analysis of published results of randomized clinical trials. J Urol 2004;171:218690, quiz 435.

225. Perlis N, Zlotta AR, Beyene J, Finelli A, Fleshner NE, Kulkarni GS. Immediate posttransurethral resection of bladder tumor intravesical chemotherapy prevents non-muscle-invasive bladder cancer recurrences: an updated meta-analysis on 2548 patients and quality-of-evidence review. Eur Urol 2013;64:421-30.

226. Abern MR, Owusu RA, Anderson MR, Rampersaud EN, Inman BA. Perioperative intravesical chemotherapy in non-muscle-invasive bladder cancer: a systematic review and metaanalysis. J Natl Compr Canc Netw 2013;11:477-84.

227. Sylvester RJ, Oosterlinck W, Holmang S, et al. Systematic Review and Individual Patient Data Meta-analysis of Randomized Trials Comparing a Single Immediate Instillation of Chemotherapy After Transurethral Resection with Transurethral Resection Alone in Patients 
with Stage pTa-pT1 Urothelial Carcinoma of the Bladder: Which Patients Benefit from the Instillation? Eur Urol 2016;69:231-44.

228. Bosschieter J, Nieuwenhuijzen JA, van Ginkel T, et al. Value of an Immediate Intravesical Instillation of Mitomycin C in Patients with Non-muscle-invasive Bladder Cancer: A Prospective Multicentre Randomised Study in 2243 patients. European Urology 2018;73:22632.

229. Bosschieter J, Nieuwenhuijzen JA, Vis AN, et al. An immediate, single intravesical instillation of mitomycin $\mathrm{C}$ is of benefit in patients with non-muscle-invasive bladder cancer irrespective of prognostic risk groups. Urol Oncol 2018;36:400.e7-.e14.

230. Bosschieter J, van Moorselaar RJA, Nieuwenhuijzen JA. An immediate, single instillation of mitomycin $\mathrm{C}$ in non-muscle invasive bladder cancer: can we define which patients do and do not benefit? Translational Andrology and Urology 2019:S346-S7.

231. Elsawy AA, El-Assmy AM, Bazeed MA, Ali-El-Dein B. The value of immediate postoperative intravesical epirubicin instillation as an adjunct to standard adjuvant treatment in intermediate and high-risk non-muscle-invasive bladder cancer: A preliminary results of randomized controlled trial. Urol Oncol 2019;37:179.e9-.e18.

232. Oosterlinck W, Decaestecker K. An immediate intravesical instillation of mitomycin C is of benefit in all prognostic risk groups with non-muscle-invasive bladder cancers. Transl Androl Urol 2018;7:S706-s9.

233. Kaasinen E, Rintala E, Hellstrom P, et al. Factors explaining recurrence in patients undergoing chemoimmunotherapy regimens for frequently recurring superficial bladder carcinoma. Eur Urol 2002;42:167-74.

234. Hendricksen K, Witjes WP, Idema JG, et al. Comparison of three schedules of intravesical epirubicin in patients with non-muscle-invasive bladder cancer. Eur Urol 2008;53:984-91.

235. Onishi T, Sugino Y, Shibahara T, Masui S, Yabana T, Sasaki T. Randomized controlled study of the efficacy and safety of continuous saline bladder irrigation after transurethral resection for the treatment of non-muscle-invasive bladder cancer. BJU Int 2017;119:276-82.

236. Mahran A, Bukavina L, Mishra K, et al. Bladder irrigation after transurethral resection of superficial bladder cancer: a systematic review of the literature. Can J Urol 2018;25:9579-84. 237. Zhou Z, Zhao S, Lu Y, et al. Meta-analysis of efficacy and safety of continuous saline bladder irrigation compared with intravesical chemotherapy after transurethral resection of bladder tumors. World J Urol 2019;37:1075-84.

238. Koga H, Kuroiwa K, Yamaguchi A, Osada Y, Tsuneyoshi M, Naito S. A Randomized Controlled Trial of Short-Term Versus Long-Term Prophylactic Intravesical Instillation Chemotherapy for Recurrence After Transurethral Resection of Ta/T1 Transitional Cell Carcinoma of the Bladder. The Journal of Urology 2004;171:153-7. 
239. Friedrich MG, Pichlmeier U, Schwaibold H, Conrad S, Huland H. Long-term intravesical adjuvant chemotherapy further reduces recurrence rate compared with short-term intravesical chemotherapy and short-term therapy with Bacillus Calmette-Guerin (BCG) in patients with non-muscle-invasive bladder carcinoma. Eur Urol 2007;52:1123-29.

240. Matsumura Y, Akaza H, Isaka S, et al. The 4th study of prophylactic intravesical chemotherapy with adriamycin in the treatment of superficial bladder cancer: the experience of the Japanese Urological Cancer Research Group for Adriamycin. Cancer Chemother Pharmacol 1992;30 Suppl:S10-4.

241. Naya Y, Mikami K, Takaha N, et al. Randomized study of intravesical pirarubicin chemotherapy with low and intermediate-risk nonmuscle-invasive bladder cancer in Japan: Comparison of a single immediate postoperative intravesical instillation with short-term adjuvant intravesical instillations after transurethral resection. Medicine (Baltimore) 2018;97:e12740. 242. Koga H, Kuroiwa K, Yamaguchi A, Osada Y, Tsuneyoshi M, Naito S. A randomized controlled trial of short-term versus long-term prophylactic intravesical instillation chemotherapy for recurrence after transurethral resection of Ta/T1 transitional cell carcinoma of the bladder. J Urol 2004;171:153-7.

243. Friedrich MG, Pichlmeier U, Schwaibold H, Conrad S, Huland H. Long-term intravesical adjuvant chemotherapy further reduces recurrence rate compared with short-term intravesical chemotherapy and short-term therapy with Bacillus Calmette-Guérin (BCG) in patients with non-muscle-invasive bladder carcinoma. Eur Urol 2007;52:1123-29.

244. Okamura K, Kinukawa T, Tsumura Y, et al. A randomized study of short-versus longterm intravesical epirubicin instillation for superficial bladder cancer. Nagoya University Urological Oncology Group. Eur Urol 1998;33:285-8; discussion 9.

245. Tolley DA, Parmar MK, Grigor KM, et al. The effect of intravesical mitomycin C on recurrence of newly diagnosed superficial bladder cancer: a further report with 7 years of follow up. J Urol 1996;155:1233-8.

246. Huncharek M, Geschwind JF, Witherspoon B, McGarry R, Adcock D. Intravesical chemotherapy prophylaxis in primary superficial bladder cancer: a meta-analysis of 3703 patients from 11 randomized trials. J Clin Epidemiol 2000;53:676-80.

247. Sylvester RJ, Oosterlinck W, Witjes JA. The Schedule and Duration of Intravesical Chemotherapy in Patients with Non-Muscle-Invasive Bladder Cancer: A Systematic Review of the Published Results of Randomized Clinical Trials. European Urology 2008;53:709-19. 248. Tabayoyong WB, Kamat AM, O'Donnell MA, et al. Systematic Review on the Utilization of Maintenance Intravesical Chemotherapy in the Management of Non-muscleinvasive Bladder Cancer. European Urology Focus 2018;4:512-21. 
249. Bohle A, Jocham D, Bock PR. Intravesical bacillus Calmette-Guerin versus mitomycin C for superficial bladder cancer: a formal meta-analysis of comparative studies on recurrence and toxicity. J Urol 2003;169:90-5.

250. Sylvester RJ, Brausi MA, Kirkels WJ, et al. Long-term efficacy results of EORTC genito-urinary group randomized phase 3 study 30911 comparing intravesical instillations of epirubicin, bacillus Calmette-Guerin, and bacillus Calmette-Guerin plus isoniazid in patients with intermediate- and high-risk stage Ta T1 urothelial carcinoma of the bladder. Eur Urol 2010;57:766-73.

251. Shelley MD, Wilt TJ, Court J, Coles B, Kynaston H, Mason MD. Intravesical bacillus Calmette-Guerin is superior to mitomycin $\mathrm{C}$ in reducing tumour recurrence in high-risk superficial bladder cancer: a meta-analysis of randomized trials. BJU Int 2004;93:485-90. 252. Malmstrom PU, Sylvester RJ, Crawford DE, et al. An individual patient data metaanalysis of the long-term outcome of randomised studies comparing intravesical mitomycin $\mathrm{C}$ versus bacillus Calmette-Guerin for non-muscle-invasive bladder cancer. Eur Urol 2009;56:24756.

253. Schmidt S, Kunath F, Coles B, et al. Intravesical Bacillus Calmette-Guerin versus mitomycin C for Ta and T1 bladder cancer. Cochrane Database Syst Rev 2020;1:Cd011935. 254. Lamm D, Persad R, Colombel M, Brausi M. Maintenance Bacillus Calmette-Guérin: The Standard of Care for the Prophylaxis and Management of Intermediate- and High-Risk NonMuscle-Invasive Bladder Cancer. European Urology Supplements 2010;9:715-34.

255. Peyton CC, Chipollini J, Azizi M, Kamat AM, Gilbert SM, Spiess PE. Updates on the use of intravesical therapies for non-muscle invasive bladder cancer: how, when and what. World J Urol 2019;37:2017-29.

256. Di Stasi SM, Giannantoni A, Stephen RL, et al. Intravesical electromotive mitomycin C versus passive transport mitomycin $\mathrm{C}$ for high-risk superficial bladder cancer: a prospective randomized study. J Urol 2003;170:777-82.

257. Di Stasi SM, Giannantoni A, Giurioli A, et al. Sequential BCG and electromotive mitomycin versus BCG alone for high-risk superficial bladder cancer: a randomised controlled trial. Lancet Oncol 2006;7:43-51.

258. Gan C, Amery S, Chatterton K, Khan MS, Thomas K, O’Brien T. Sequential bacillus Calmette-Gu\&\#xe9;rin/Electromotive Drug Administration of Mitomycin C as the Standard Intravesical Regimen in High-risk Nonmuscle Invasive Bladder Cancer: 2-Year Outcomes. Journal of Urology 2016;195:1697-703.

259. Colombo R, Da Pozzo LF, Salonia A, et al. Multicentric study comparing intravesical chemotherapy alone and with local microwave hyperthermia for prophylaxis of recurrence of superficial transitional cell carcinoma. J Clin Oncol 2003;21:4270-6. 
260. Colombo R, Salonia A, Leib Z, Pavone-Macaluso M, Engelstein D. Long-term outcomes of a randomized controlled trial comparing thermochemotherapy with mitomycin-C alone as adjuvant treatment for non-muscle-invasive bladder cancer (NMIBC). BJU Int 2011;107:912-8. 261. Arends TJ, Nativ O, Maffezzini M, et al. Results of a Randomised Controlled Trial Comparing Intravesical Chemohyperthermia with Mitomycin C Versus Bacillus CalmetteGuerin for Adjuvant Treatment of Patients with Intermediate- and High-risk Non-Muscleinvasive Bladder Cancer. Eur Urol 2016;69:1046-52.

262. Morales A, Eidinger D, Bruce AW. Intracavitary Bacillus Calmette-Guerin in the treatment of superficial bladder tumors. J Urol 1976;116:180-3.

263. Lamm DL. Bacillus Calmette-Guerin immunotherapy for bladder cancer. The Journal of urology 1985;134:40-7.

264. Herr HW, Pinsky CM, Whitmore WF, Jr., Sogani PG, Oettgen HF, Melamed MR. Experience with intravesical bacillus Calmette-Guerin therapy of superficial bladder tumors. Urology 1985;25:119-23.

265. Nseyo UO, Lamm DL. Immunotherapy of bladder cancer. Semin Surg Oncol 1997;13:342-9.

266. Krege S, Giani G, Meyer R, Otto T, Rubben H. A randomized multicenter trial of adjuvant therapy in superficial bladder cancer: transurethral resection only versus transurethral resection plus mitomycin $\mathrm{C}$ versus transurethral resection plus bacillus Calmette-Guerin. Participating Clinics. J Urol 1996;156:962-6.

267. Melekos MD, Chionis H, Pantazakos A, Fokaefs E, Paranychianakis G, Dauaher H. Intravesical bacillus Calmette-Guerin immunoprophylaxis of superficial bladder cancer: results of a controlled prospective trial with modified treatment schedule. J Urol 1993;149:744-8. 268. Herr HW. Transurethral resection and intravesical therapy of superficial bladder tumors. Urol Clin North Am 1991;18:525-8.

269. Huncharek M, Kupelnick B. Impact of intravesical chemotherapy versus BCG immunotherapy on recurrence of superficial transitional cell carcinoma of the bladder: metaanalytic reevaluation. Am J Clin Oncol 2003;26:402-7.

270. Sylvester RJ, van der MA, Lamm DL. Intravesical bacillus Calmette-Guerin reduces the risk of progression in patients with superficial bladder cancer: a meta-analysis of the published results of randomized clinical trials. The Journal of urology 2002;168:1964-70.

271. Bohle A, Bock PR. Intravesical bacille Calmette-Guerin versus mitomycin C in superficial bladder cancer: formal meta-analysis of comparative studies on tumor progression. Urology 2004;63:682-6; discussion 6-7.

272. Sylvester RJ, van der Meijden APM, Witjes JA, Kurth K. Bacillus calmette-guerin versus chemotherapy for the intravesical treatment of patients with carcinoma in situ of the bladder: a 
meta-analysis of the published results of randomized clinical trials. The Journal of urology 2005; 174:86-92.

273. Herr HW, Laudone VP, Badalament RA, et al. Bacillus Calmette-Guérin therapy alters the progression of superficial bladder cancer. J Clin Oncol 1988;6:1450-5.

274. Herr HW, Pinsky CM, Whitmore WF, Jr., Oettgen HF, Melamed MR. Effect of intravesical Bacillus Calmette-Guerin (BCG) on carcinoma in situ of the bladder. Cancer 1983;51:1323-6.

275. Lamm DL, Blumenstein BA, Crissman JD, et al. MAINTENANCE BACILLUS CALMETTE-GUERIN IMMUNOTHERAPY FOR RECURRENT TA, T1 AND CARCINOMA IN SITU TRANSITIONAL CELL CARCINOMA OF THE BLADDER: A RANDOMIZED SOUTHWEST ONCOLOGY GROUP STUDY. The Journal of Urology 2000;163:1124-9. 276. Oddens J, Brausi M, Sylvester R, et al. Final results of an EORTC-GU cancers group randomized study of maintenance bacillus Calmette-Guérin in intermediate- and high-risk Ta, T1 papillary carcinoma of the urinary bladder: one-third dose versus full dose and 1 year versus 3 years of maintenance. European urology 2013;63:462-72.

277. Martínez-Piñeiro JA, Flores N, Isorna S, et al. Long-term followup of a randomized prospective trial comparing a standard $81 \mathrm{mg}$ dose of intravesical bacille Calmette-Guérin with a reduced dose of $27 \mathrm{mg}$ in superficial bladder cancer. BJU international 2002;89:671-80.

278. Grimm MO, van der Heijden AG, Colombel M, et al. Treatment of High-grade Nonmuscle-invasive Bladder Carcinoma by Standard Number and Dose of BCG Instillations Versus Reduced Number and Standard Dose of BCG Instillations: Results of the European Association of Urology Research Foundation Randomised Phase III Clinical Trial "NIMBUS". Eur Urol 2020 .

279. Boehm BE, Cornell JE, Wang H, Mukherjee N, Oppenheimer JS, Svatek RS. Efficacy of bacillus Calmette-Guérin Strains for Treatment of Nonmuscle Invasive Bladder Cancer: A Systematic Review and Network Meta-Analysis. The Journal of Urology 2017;198:503-10. 280. D'Andrea D, Soria F, Abufaraj M, et al. Comparative Effectiveness of Intravesical BCGTice and BCG-Moreau in Patients With Non-muscle-invasive Bladder Cancer. Clinical Genitourinary Cancer 2020;18:20-5.e2.

281. Rentsch CA, Birkhäuser FD, Biot C, et al. Bacillus Calmette-Guérin Strain Differences Have an Impact on Clinical Outcome in Bladder Cancer Immunotherapy. European Urology 2014;66:677-88.

282. Sengiku A, Ito M, Miyazaki Y, Sawazaki H, Takahashi T, Ogura K. A Prospective Comparative Study of Intravesical Bacillus Calmette-Guérin Therapy with the Tokyo or Connaught Strain for Nonmuscle Invasive Bladder Cancer. The Journal of Urology 2013;190:504. 
283. Vegt PDJ, Witjes JA, Witjes WPJ, et al. Original Articles: Bladder Cancer: A Randomized Study of Intravesical Mitomycin C, Bacillus Calmette-Guerin Tice and Bacillus Calmette-Guerin RIVM Treatment in pTa-pT1 Papillary Carcinoma and Carcinoma in Situ of the Bladder. The Journal of Urology 1995;153:929-33.

284. Witjes JA, Dalbagni G, Karnes RJ, et al. The efficacy of BCG TICE and BCG Connaught in a cohort of 2,099 patients with T1G3 non-muscle-invasive bladder cancer. Urol Oncol 2016;34:484.e19-.e25.

285. van der Meijden APM, Sylvester RJ, Oosterlinck W, Hoeltl W, Bono AV. Maintenance Bacillus Calmette-Guerin for Ta T1 Bladder Tumors Is Not Associated with Increased Toxicity: Results from a European Organisation for Research and Treatment of Cancer Genito-Urinary Group Phase III Trial. European Urology 2003;44:429-34.

286. Poletajew S, Krajewski W, Adamowicz J, Radziszewski P. A systematic review of preventive and therapeutic options for symptoms of cystitis in patients with bladder cancer receiving intravesical bacillus Calmette-Guerin immunotherapy. Anticancer Drugs 2019;30:51722.

287. Mathes J, Todenhofer T. Managing Toxicity of Intravesical Therapy. Eur Urol Focus 2018;4:464-7.

288. Lamm DL, Van Der Meijden APM, Morales A, et al. Incidence and Treatment of Complications of Bacillus Calmette-Guerin Intravesical Therapy in Superficial Bladder Cancer. The Journal of Urology 1992;147:596-600.

289. Colombel M, Saint F, Chopin D, Malavaud B, Nicolas L, Rischmann P. The effect of ofloxacin on bacillus calmette-guerin induced toxicity in patients with superficial bladder cancer: results of a randomized, prospective, double-blind, placebo controlled, multicenter study. J Urol 2006;176:935-9.

290. van der Meijden AP, Brausi M, Zambon V, et al. Intravesical instillation of epirubicin, bacillus Calmette-Guerin and bacillus Calmette-Guerin plus isoniazid for intermediate and highrisk Ta, T1 papillary carcinoma of the bladder: a European Organization for Research and Treatment of Cancer genito-urinary group randomized phase III trial. The Journal of urology 2001;166:476-81.

291. Griffin JG, Holzbeierlein J. Side effects of perioperative intravesical treatment and treatment strategies for these side effects. Urol Clin North Am 2013;40:197-210.

292. Rischmann P, Desgrandchamps F, Malavaud B, Chopin DK. BCG intravesical instillations: recommendations for side-effects management. Eur Urol 2000;37 Suppl 1:33-6.

293. Koya MP, Simon MA, Soloway MS. Complications of intravesical therapy for urothelial cancer of the bladder. J Urol 2006;175:2004-10. 
294. Witjes JA, Palou J, Soloway M, et al. Clinical Practice Recommendations for the Prevention and Management of Intravesical Therapy-Associated Adverse Events. European Urology Supplements 2008;7:667-74.

295. Hemdan T, Johansson R, Jahnson S, Hellstrom P, Tasdemir I, Malmstrom PU. 5-Year outcome of a randomized prospective study comparing bacillus Calmette-Guerin with epirubicin and interferon-alpha2b in patients with T1 bladder cancer. J Urol 2014;191:1244-9.

296. Herr HW, Dalbagni G. Defining bacillus Calmette-Guerin refractory superficial bladder tumors. J Urol 2003;169:1706-8.

297. Lerner SP, Tangen CM, Sucharew H, Wood D, Crawford ED. Failure to achieve a complete response to induction BCG therapy is associated with increased risk of disease worsening and death in patients with high-risk non-muscle invasive bladder cancer. Urol Oncol 2009;27:155-9.

298. Solsona E, Iborra I, Dumont R, Rubio-Briones J, Casanova J, Almenar S. The 3-month clinical response to intravesical therapy as a predictive factor for progression in patients with high-risk superficial bladder cancer. J Urol 2000;164:685-9.

299. Herr HW, Sogani PC. Does early cystectomy improve the survival of patients with highrisk superficial bladder tumors? J Urol 2001;166:1296-9.

300. Jäger W, Thomas C, Haag S, et al. Early vs delayed radical cystectomy for 'high-risk' carcinoma not invading bladder muscle: delay of cystectomy reduces cancer-specific survival. BJU International 2011;108:E284-E8.

301. Balar AV, Kulkarni GS, Uchio EM, et al. Keynote 057: Phase II trial of Pembrolizumab (pembro) for patients (pts) with high-risk (HR) nonmuscle invasive bladder cancer (NMIBC) unresponsive to bacillus calmette-guérin (BCG). Journal of Clinical Oncology 2019;37:350-. 302. Boorjian SA, Alemozaffar M, Konety BR, et al. Intravesical nadofaragene firadenovec gene therapy for BCG-unresponsive non-muscle-invasive bladder cancer: a single-arm, openlabel, repeat-dose clinical trial. Lancet Oncol 2020.

303. Steinberg G, Bahnson R, Brosman S, Middleton R, Wajsman Z, Wehle M. Efficacy and safety of valrubicin for the treatment of Bacillus Calmette-Guerin refractory carcinoma in situ of the bladder. The Valrubicin Study Group. J Urol 2000;163:761-7.

304. Dinney CP, Greenberg RE, Steinberg GD. Intravesical valrubicin in patients with bladder carcinoma in situ and contraindication to or failure after bacillus Calmette-Guerin. Urol Oncol 2013;31:1635-42.

305. Laudano MA, Barlow LJ, Murphy AM, et al. Long-term Clinical Outcomes of a Phase I Trial of Intravesical Docetaxel in the Management of Non-muscle-invasive Bladder Cancer Refractory to Standard Intravesical Therapy. Urology 2010;75:134-7. 
306. Barlow LJ, McKiernan JM, Benson MC. Long-term survival outcomes with intravesical docetaxel for recurrent nonmuscle invasive bladder cancer after previous bacillus CalmetteGuerin therapy. J Urol 2013;189:834-9.

307. Dalbagni G, Russo P, Sheinfeld J, et al. Phase I trial of intravesical gemcitabine in bacillus Calmette-Guerin-refractory transitional-cell carcinoma of the bladder. J Clin Oncol 2002;20:3193-8.

308. Skinner EC, Goldman B, Sakr WA, et al. SWOG S0353: Phase II trial of intravesical gemcitabine in patients with nonmuscle invasive bladder cancer and recurrence after 2 prior courses of intravesical bacillus Calmette-Guerin. J Urol 2013;190:1200-4.

309. Morales A, Herr H, Steinberg G, et al. Efficacy and safety of MCNA in patients with nonmuscle invasive bladder cancer at high-risk for recurrence and progression after failed treatment with bacillus Calmette-Guérin. J Urol 2015;193:1135-43.

310. McKiernan JM, Holder DD, Ghandour RA, et al. Phase II trial of intravesical nanoparticle albumin bound paclitaxel for the treatment of nonmuscle invasive urothelial carcinoma of the bladder after bacillus Calmette-Guérin treatment failure. J Urol 2014;192:16338.

311. Racioppi M, Di Gianfrancesco L, Ragonese M, Palermo G, Sacco E, Bassi PF. ElectroMotive drug administration (EMDA) of Mitomycin $\mathrm{C}$ as first-line salvage therapy in high-risk "BCG failure" non muscle invasive bladder cancer: 3 years followup outcomes. BMC Cancer 2018;18:1224.

312. Juvet T, Mari A, Lajkosz K, et al. Sequential administration of Bacillus Calmette-Guerin (BCG) and Electromotive Drug Administration (EMDA) of mitomycin C (MMC) for the treatment of high-grade nonmuscle invasive bladder cancer after BCG failure. Urol Oncol 2020. 313. Tan WS, Panchal A, Buckley L, et al. Radiofrequency-induced Thermo-chemotherapy Effect Versus a Second Course of Bacillus Calmette-Guérin or Institutional Standard in Patients with Recurrence of Non-muscle-invasive Bladder Cancer Following Induction or Maintenance Bacillus Calmette-Guérin Therapy (HYMN): A Phase III, Open-label, Randomised Controlled Trial. Eur Urol 2019;75:63-71.

314. Lightfoot AJ, Breyer BN, Rosevear HM, Erickson BA, Konety BR, O'Donnell MA. Multi-institutional analysis of sequential intravesical gemcitabine and mitomycin $\mathrm{C}$ chemotherapy for non-muscle invasive bladder cancer. Urol Oncol 2014;32:35.e15-9.

315. Steinberg RL, Thomas LJ, O'Donnell MA, Nepple KG. Sequential Intravesical Gemcitabine and Docetaxel for the Salvage Treatment of Non-Muscle Invasive Bladder Cancer. Bladder Cancer 2015;1:65-72.

316. Milbar N, Kates M, Chappidi MR, et al. Oncological Outcomes of Sequential Intravesical Gemcitabine and Docetaxel in Patients with Non-Muscle Invasive Bladder Cancer. Bladder Cancer 2017;3:293-303. 
317. Steinberg RL, Thomas LJ, Brooks N, et al. Multi-Institution Evaluation of Sequential Gemcitabine and Docetaxel as Rescue Therapy for Nonmuscle Invasive Bladder Cancer. J Urol 2020;203:902-9.

318. Breyer BN, Whitson JM, Carroll PR, Konety BR. Sequential intravesical gemcitabine and mitomycin $\mathrm{C}$ chemotherapy regimen in patients with non-muscle invasive bladder cancer. Urologic Oncology: Seminars and Original Investigations 2010;28:510-4.

319. Cockerill PA, Knoedler JJ, Frank I, Tarrell R, Karnes RJ. Intravesical gemcitabine in combination with mitomycin $\mathrm{C}$ as salvage treatment in recurrent non-muscle-invasive bladder cancer. BJU Int 2016;117:456-62.

320. Joudi FN, Smith BJ, O'Donnell MA. Final results from a national multicenter phase II trial of combination bacillus Calmette-Guerin plus interferon alpha-2B for reducing recurrence of superficial bladder cancer. Urol Oncol 2006;24:344-8.

321. Gallagher BL, Joudi FN, Maymi JL, O'Donnell MA. Impact of previous bacille CalmetteGuerin failure pattern on subsequent response to bacille Calmette-Guerin plus interferon intravesical therapy. Urology 2008;71:297-301.

322. Fradet Y, Bellmunt J, Vaughn DJ, et al. Randomized phase III KEYNOTE-045 trial of pembrolizumab versus paclitaxel, docetaxel, or vinflunine in recurrent advanced urothelial cancer: results of $>2$ years of followup. Ann Oncol 2019.

323. Shore ND, Boorjian SA, Canter DJ, et al. Intravesical rAd-IFN $\alpha / S y n 3$ for Patients With High-Grade, Bacillus Calmette-Guerin-Refractory or Relapsed Non-Muscle-Invasive Bladder Cancer: A Phase II Randomized Study. J Clin Oncol 2017;35:3410-6.

324. Dickstein R, Wu N, Cowan B, et al. LBA27 PHASE 3 STUDY OF VICINIUM IN BCGUNRESPONSIVE NON-MUSCLE INVASIVE BLADDER CANCER: INITIAL RESULTS. Journal of Urology 2018;199:e1167-e.

325. Chamie K, Lee JH, Rock A, Rhode PR, Soon-Shiong P. Preliminary phase 2 clinical results of IL-15R $\alpha \mathrm{Fc}$ superagonist $\mathrm{N}-803$ with $\mathrm{BCG}$ in BCG-unresponsive non-muscle invasive bladder cancer (NMIBC) patients. Journal of Clinical Oncology 2019;37:4561-. 326. Meng MV, Gschwend JE, Shore N, Grossfeld GD, Mostafid H, Black PC. Emerging Immunotherapy Options for bacillus Calmette-Guerin Unresponsive Nonmuscle Invasive Bladder Cancer. J Urol 2019;202:1111-9.

327. Kamat AM, Shore N, Hahn N, et al. KEYNOTE-676: Phase III study of BCG and pembrolizumab for persistent/recurrent high-risk NMIBC. Future Oncology 2020;16:507-16. 328. Weiss C, Wolze C, Engehausen DG, et al. Radiochemotherapy after transurethral resection for high-risk $\mathrm{T} 1$ bladder cancer: an alternative to intravesical therapy or early cystectomy? J Clin Oncol 2006;24:2318-24. 
329. Giacalone NJ, Shipley WU, Clayman RH, et al. Long-term Outcomes After Bladderpreserving Tri-modality Therapy for Patients with Muscle-invasive Bladder Cancer: An Updated Analysis of the Massachusetts General Hospital Experience. Eur Urol 2017;71:952-60.

330. Chehroudi AC, Black PC. Emerging intravesical therapies for the management of bacillus Calmette-Guérin (BCG)-unresponsive non-muscle-invasive bladder cancer: Charting a path forward. Canadian Urological Association journal = Journal de l'Association des urologues du Canada 2020;14:204-13.

331. Ourfali S, Ohannessian R, Fassi-Fehri H, Pages A, Badet L, Colombel M. Recurrence Rate and Cost Consequence of the Shortage of Bacillus Calmette-Guérin Connaught Strain for Bladder Cancer Patients. European Urology Focus 2019.

332. Davies BJ, Hwang TJ, Kesselheim AS. Ensuring Access to Injectable Generic Drugs The Case of Intravesical BCG for Bladder Cancer. N Engl J Med 2017;376:1401-3.

333. Chou R, Selph S, Buckley DI, et al. Intravesical Therapy for the Treatment of Nonmuscle Invasive Bladder Cancer: A Systematic Review and Meta-Analysis. J Urol 2017;197:1189-99. 334. Shelley MD, Mason MD, Kynaston H. Intravesical therapy for superficial bladder cancer: a systematic review of randomised trials and meta-analyses. Cancer Treat Rev 2010;36:195-205. 335. Koch GE, Luckenbaugh AN, Chang SS. High-Risk Non-Muscle Invasive Bladder Cancer: Selecting the Appropriate Patient for Timely Cystectomy. Urology 2020.

336. Hautmann RE, Volkmer BG, Gust K. Quantification of the survival benefit of early versus deferred cystectomy in high-risk non-muscle invasive bladder cancer (T1 G3). World J Urol 2009;27:347-51.

337. Stein JP, Lieskovsky G, Cote R, et al. Radical cystectomy in the treatment of invasive bladder cancer: long-term results in 1,054 patients. J Clin Oncol 2001;19:666-75.

338. Stimson CJ, Chang SS, Barocas DA, et al. Early and late perioperative outcomes following radical cystectomy: 90 -day readmissions, morbidity and mortality in a contemporary series. J Urol 2010;184:1296-300.

339. Klaassen Z, Kamat AM, Kassouf W, et al. Treatment Strategy for Newly Diagnosed T1 High-grade Bladder Urothelial Carcinoma: New Insights and Updated Recommendations. Eur Urol 2018;74:597-608.

340. Catto JWF, Gordon K, Collinson M, et al. Radical Cystectomy Against Intravesical BCG for High-Risk High-Grade Nonmuscle Invasive Bladder Cancer: Results From the Randomized Controlled BRAVO-Feasibility Study. J Clin Oncol 2020:Jco2001665.

341. Halling KC, King W, Sokolova IA, et al. A comparison of cytology and fluorescence in situ hybridization for the detection of urothelial carcinoma. J Urol 2000;164:1768-75.

342. Herr HW, Wartinger DD, Fair WR, Oettgen HF. Bacillus Calmette-Guerin Therapy for Superficial Bladder Cancer: A 10-Year Followup. The Journal of Urology 1992;147:1020-3. 
343. Mariappan P, Smith G. A surveillance schedule for G1Ta bladder cancer allowing efficient use of check cystoscopy and safe discharge at 5 years based on a 25-year prospective database. J Urol 2005;173:1108-11.

344. Millan-Rodriguez F, Chechile-Toniolo G, Salvador-Bayarri J, Huguet-Perez J, VicenteRodriguez J. Upper urinary tract tumors after primary superficial bladder tumors: prognostic factors and risk groups. J Urol 2000;164:1183-7.

345. Lin N, Wu YP, Lin YZ, et al. Risk factors for upper tract urothelial recurrence following local excision of bladder cancer. Cancer Med 2018;7:4098-103.

346. Golabesk T, Palou J, Rodriguez O, et al. Long-term Bladder and Upper Urinary Tract Followup Recurrence and Progression Rates of G1-2 Non-muscle-invasive Urothelial Carcinoma of the Bladder. Urology 2017;100:145-50.

347. Brummelhuis ISG, Witjes JA, Messing EM. The conundrum of recurrent low-grade tumours: to treat or to observe? Current Opinion in Urology 2018;28:557-62.

348. Green DA, Rink M, Cha EK, et al. Cost-effective treatment of low-risk carcinoma not invading bladder muscle. BJU Int 2013;111:E78-84.

349. Donat SM, North A, Dalbagni G, Herr HW. Efficacy of office fulguration for recurrent low grade papillary bladder tumors less than $0.5 \mathrm{~cm}$. J Urol 2004;171:636-9.

350. Herr HW, Donat SM, Reuter VE. Management of Low Grade Papillary Bladder Tumors. The Journal of Urology 2007;178:1201-5.

351. Kassouf W, Traboulsi SL, Schmitz-Drager B, et al. Followup in non-muscle-invasive bladder cancer-International Bladder Cancer Network recommendations. Urol Oncol 2016;34:460-8.

352. Marcq G, Henon F, Ouzaid I, Fantoni JC, Hermieu JF, Xylinas E. Active surveillance for non-muscle invasive bladder cancer. Transl Androl Urol 2019;8:54-60. 